\begin{tabular}{|c|l|}
\hline Title & The mandal o of Legendrian dual ities for pseudo-spheres in Lorentz-Minkowski space and "flat" spacelike surfaces \\
\hline Author(s) & Izumiya, Shyuichi; Saji, Kentaro \\
\hline Citation & Hokkaido University Preprint Series in Mathematics, 947, 1-35 \\
\hline Issue Date & 2009-9-30 \\
\hline DOI & 10.14943/84094 \\
\hline Doc URL & http://hdl.handle.net/2115/69754 \\
\hline Type & bulletin (article) \\
\hline File Information & pre947.pdf \\
\hline
\end{tabular}

Instructions for use 


\title{
The mandala of Legendrian dualities for pseudo-spheres in Lorentz-Minkowski space and "flat" spacelike surfaces.
}

\author{
Shyuichi Izumiya and Kentaro Saji
}

September 23, 2009

\begin{abstract}
Using the Legnedrian duarities between surfaces in pseudo-spheres in Lorentz-Minkowski 4-space, we study various kind of flat surfaces in pseudo-spheres. We consider a surface in the pseudo-sphere and its dual surface. Flatness of a surface is defined by the degeneracy of the dual surface similar to the case for the Gauss map of a flat surface in the Euclidean space. We study singularities of these flat surfaces and dualities of singularities.
\end{abstract}

\section{Introduction}

It has been shown in [25] that a theorem of Legendrian dualities for pseudo-spheres in LorentzMinkowski space which gives a commutative diagram between contact manifolds defined by the dual relations. This theorem has been generalized into psuedo-spheres in semi-Euclidean space with genral index in [10]. Such a commjutative diagram is called a mandala of Legendrian dualities now [10, 26]. The mandala of Legednrian dualities is very useful for the study of the differential geometry on submanifolds in pseudo-spheres. Especially, it works well even for spacelike hypersurfaces in the lightcone where the induced metric is degenerate[25].

In this paper we consider various kinds of flatness of surfaces in pseudo-spheres in LorentzMinkowski space. In Euclidean space, a flat surface is characterized by the degeneracy of the Gauss map. For example, a surface is a part of a plane if the Gauss map is constant. Moreover, a surface is a developable surface if the image of the Gauss map is a point or a curve (i.e., all points of the surface are singularities of the Gauss map). We remark that the dual surface of a surface plays a similar roles as the Gauss map of the surface $[24,31]$. Accoreding to the these facts on the Euclidean case, the Legedrian dual of a surface in pseudo-sphere is considered to be a kind of the Gauss map of the surface. In this sense a surface in a pseudo-sphere is "flat" if the Legendrian dual is singular at any point of the surface. Especially, we consider the case when the Legendrian dual is a curve in a pseudo-sphere. In [22] we have studied a surface in Hyperbolic space whose lightcone dual is a curve.In this case the suface is called a horo-flat surface. Moreover, such surfaces are one-parameter families of horo-cycles. Therefore, we call it a horo-flat horocyclic surface. Horo-flat surfaces are "flat"surfaces in the sense of a new geometry in Hyperbolic space[5, 6, 17, 18, 19, 22] which is called "Horospherical Geometry".

2000 Mathematics Subject classification. 53A35, 57R45, 53C42

Keywords and Phrase. Legendrian duality, Horo-spherical geometry, Horo-flat surfaces, Singularities, Lorentz-Minkowski space, Pseudo-spheres 
In this paper we consider surfaces with similar properties as horo-flat horo-cyclic surfaces in other pseudo-spheres. These surface can be obatined by the aid of the mandala of Legendrian dualities. One of the main results in this paper is to give classifications of the singularities of these surfaces and show dualities among singularities. Therefore, the mandala of Legedrian dualities still remains on the singularities level. As a consequence, these surfaces are frontals which are the projection images of istropic surfaces in the total contact manifold of a Legenrian fibration. If the istropic surface is a Legendrian surface, the frontal is called a wave front (or, simply a front).

Singularities of wave fronts have been originally investigated by Zakalyukin[34, 35]. See [2] for the detail. He has shown that generic singularities of wave front surfaces are the cuspidal edge and the swallowtail. It is known that generic singularities of frontal surfaces are cuspidal cross cap in addition to the above two fronts $[14,15]$.

Here, the cuspidal edge is a map germ $\left(\left(\mathbb{R}^{2} ; u, v\right), \mathbf{0}\right) \rightarrow\left(\mathbb{R}^{3}, \mathbf{0}\right)$ defined by $(u, v) \mapsto\left(u, v^{2}, v^{3}\right)$ at the origin, the swallowtail is a map germ $\left(\left(\mathbb{R}^{2} ; u, v\right), \mathbf{0}\right) \rightarrow\left(\mathbb{R}^{3}, \mathbf{0}\right)$ defined by $(u, v) \mapsto$ $\left(u, 3 v^{4}+u^{2} v, 4 v^{3}+2 u v\right)$ and the cuspidal cross cap is a map germ $\left(\left(\mathbb{R}^{2} ; u, v\right), \mathbf{0}\right) \rightarrow\left(\mathbb{R}^{3}, \mathbf{0}\right)$ defined by $(u, v) \mapsto\left(u, v^{2}, u v^{3}\right)$ at the origin. Furthermore, the dual surfaces have the more degenerate singularities which called cuspidal lips or cuspidal beaks and cuspidal butterfly. The cuspidal lips (resp. cuspidal beaks) is a map germ $\left(\left(\mathbb{R}^{2} ; u, v\right), \mathbf{0}\right) \rightarrow\left(\mathbb{R}^{3}, \mathbf{0}\right)$ defined by $(u, v) \mapsto\left(u,-2 v^{3}+u^{2} v, 3 v^{4}-u^{2} v^{2}\right)\left(\operatorname{resp} .(u, v) \mapsto\left(u,-2 v^{3}-u^{2} v, 3 v^{4}-u^{2} v^{2}\right)\right)$. The cuspidal butterfly is a map germ $\left(\left(\mathbb{R}^{2} ; u, v\right), \mathbf{0}\right) \rightarrow\left(\mathbb{R}^{3}, \mathbf{0}\right)$ defined by $(u, v) \mapsto\left(u, 5 v^{4}+2 u v, 4 v^{5}+u v^{2}-u^{2}\right)$. We can draw the pictures of these singularities here.

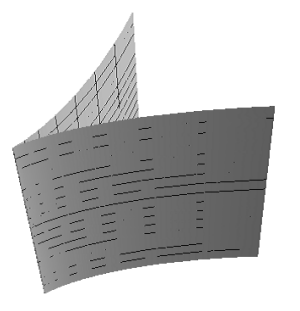

cuspidal edge

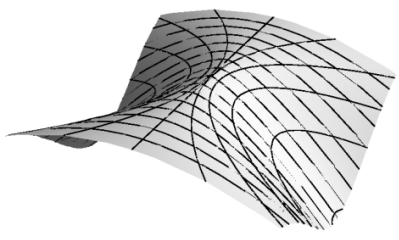

cuspidal lips

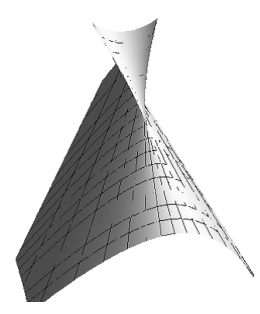

swallowtail

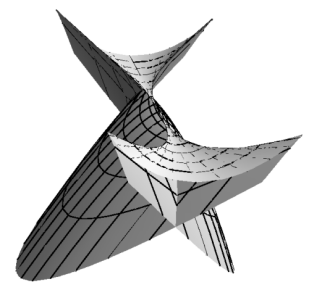

cuspidal beaks

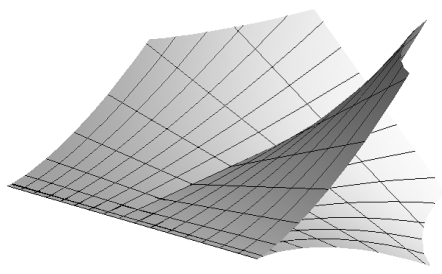

cuspidal cross cap

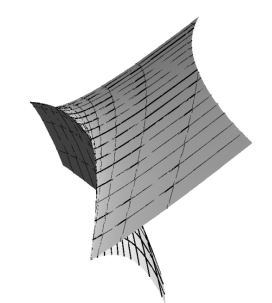

cuspidal butterfly

Figure 1.

We study singularities of maps up to $\mathcal{A}$-equivalence of map germs. Here, map germs $f_{1}, f_{2}$ : $\left(\mathbb{R}^{2}, \mathbf{0}\right) \rightarrow\left(\mathbb{R}^{3}, \mathbf{0}\right)$ are $\mathcal{A}$-equivalent if there exist diffeomorphic map germs $\phi_{1}:\left(\mathbb{R}^{2}, \mathbf{0}\right) \rightarrow\left(\mathbb{R}^{2}, \mathbf{0}\right)$ and $\phi_{2}\left(\mathbb{R}^{3}, \mathbf{0}\right) \rightarrow\left(\mathbb{R}^{3}, \mathbf{0}\right)$ such that $f_{1} \circ \phi_{2}=\phi_{1} \circ f_{2}$ holds. In Section 8 we give criteria to detect the map-germs in the above list of frontals. In order to give classifications of "flat" surfaces we construct a basic Lorentzian invariant in Section 6. We give characterizations of the above singularities of our surfaces by using such invariants (cf., Therorems 8.6, 8.8, 8.9, 8.10, 8.11, 8.13 and 8.14). 
On the other hand, there are many investigations on linear Weingarten surfaces in pseudospheres $([1,8,11,12,27])$. The mandala of Legendrian duality is deeply related to linear Weingarten surfaces. By using the mandala of Legendrian dualities, we can unify the notion of linear Weingarten surfaces in different pseudo-spheres. (cf. Theorem 5.3)

We assume throughout the whole paper that all the maps and manifolds are $C^{\infty}$ unless the contrary is explicitly stated.

\section{Basic concepts and notations}

In this section we prepare basic notions on Minkowski space. For detailed properties, see [29]. Let $\mathbb{R}^{n+1}=\left\{\left(x_{0}, x_{1}, \ldots, x_{n}\right) \mid x_{i} \in \mathbb{R}, i=0,1, \ldots, n\right\}$ be an $(n+1)$-dimensional vector space. For any vectors $\boldsymbol{x}=\left(x_{0}, \ldots, x_{n}\right), \boldsymbol{y}=\left(y_{0}, \ldots, y_{n}\right)$ in $\mathbb{R}^{n+1}$, the pseudo scalar product of $\boldsymbol{x}$ and $\boldsymbol{y}$ is defined by $\langle\boldsymbol{x}, \boldsymbol{y}\rangle=-x_{0} y_{0}+\sum_{i=1}^{n} x_{i} y_{i}$. The space $\left(\mathbb{R}^{n+1},\langle\rangle,\right)$ is called Minkowski $(n+1)$-space and denoted by $\mathbb{R}_{1}^{n+1}$.

We say that a vector $\boldsymbol{x}$ in $\mathbb{R}^{n+1} \backslash\{\mathbf{0}\}$ is spacelike, lightlike or timelike if $\langle\boldsymbol{x}, \boldsymbol{x}\rangle>0,=0$ or $<0$ respectively. The norm of the vector $\boldsymbol{x} \in \mathbb{R}^{n+1}$ is defined by $\|\boldsymbol{x}\|=\sqrt{|\langle\boldsymbol{x}, \boldsymbol{x}\rangle|}$. Given a vector $\boldsymbol{n} \in \mathbb{R}_{1}^{n+1}$ and a real number $c$, the hyperplane with pseudo normal $\boldsymbol{n}$ is given by

$$
H P(\boldsymbol{n}, c)=\left\{\boldsymbol{x} \in \mathbb{R}_{1}^{n+1} \mid\langle\boldsymbol{x}, \boldsymbol{n}\rangle=c\right\}
$$

We say that $H P(\boldsymbol{n}, c)$ is a spacelike, timelike or lightlike hyperplane if $\boldsymbol{n}$ is timelike, spacelike or lightlike respectively.

We have the following three kinds of pseudo-spheres in $\mathbb{R}_{1}^{n+1}$ : The hyperbolic $n$-space is defined by

$$
H^{n}(-1)=\left\{\boldsymbol{x} \in \mathbb{R}_{1}^{n+1} \mid\langle\boldsymbol{x}, \boldsymbol{x}\rangle=-1\right\}
$$

the de Sitter n-space by

$$
S_{1}^{n}=\left\{\boldsymbol{x} \in \mathbb{R}_{1}^{n+1} \mid\langle\boldsymbol{x}, \boldsymbol{x}\rangle=1\right\}
$$

and the (open) lightcone by

$$
L C^{*}=\left\{\boldsymbol{x} \in \mathbb{R}_{1}^{n+1} \backslash\{\mathbf{0}\} \mid\langle\boldsymbol{x}, \boldsymbol{x}\rangle=0\right\} .
$$

For any $\boldsymbol{x}^{1}, \boldsymbol{x}^{2}, \ldots, \boldsymbol{x}^{n} \in \mathbb{R}_{1}^{n+1}$, we define a vector $\boldsymbol{x}^{1} \wedge \boldsymbol{x}^{2} \wedge \cdots \wedge \boldsymbol{x}^{n}$ by

$$
\boldsymbol{x}^{1} \wedge \boldsymbol{x}^{2} \wedge \cdots \wedge \boldsymbol{x}^{n}=\left|\begin{array}{cccc}
-\boldsymbol{e}_{0} & \boldsymbol{e}_{1} & \cdots & \boldsymbol{e}_{n} \\
x_{0}^{1} & x_{1}^{1} & \cdots & x_{n}^{1} \\
x_{0}^{2} & x_{1}^{2} & \cdots & x_{n}^{2} \\
\vdots & \vdots & \cdots & \vdots \\
x_{0}^{n} & x_{1}^{n} & \cdots & x_{n}^{n}
\end{array}\right|
$$

where $\boldsymbol{e}_{0}, \boldsymbol{e}_{1}, \ldots, \boldsymbol{e}_{n}$ is the canonical basis of $\mathbb{R}_{1}^{n+1}$ and $\boldsymbol{x}^{i}=\left(x_{0}^{i}, x_{1}^{i}, \ldots, x_{n}^{i}\right)$. We can easily check that

$$
\left\langle\boldsymbol{x}, \boldsymbol{x}^{1} \wedge \boldsymbol{x}^{2} \wedge \cdots \wedge \boldsymbol{x}^{n}\right\rangle=\operatorname{det}\left(\boldsymbol{x}, \boldsymbol{x}^{1}, \ldots, \boldsymbol{x}^{n}\right),
$$

so that $\boldsymbol{x}^{1} \wedge \boldsymbol{x}^{2} \wedge \cdots \wedge \boldsymbol{x}^{n}$ is pseudo orthogonal to any $\boldsymbol{x}^{i}(i=1, \ldots, n)$. 


\section{A mandala of Legendrian dualities for pseudo-spheres}

We now review some properties of contact manifolds and Legendrian submanifolds. Let $N$ be a $(2 n+1)$-dimensional smooth manifold and $K$ be a tangent hyperplane field on $N$. Locally such a field is defined as the field of zeros of a 1-form $\alpha$. The tangent hyperplane field $K$ is nondegenerate if $\alpha \wedge(d \alpha)^{n} \neq 0$ at any point of $N$. We say that $(N, K)$ is a contact manifold if $K$ is a non-degenerate hyperplane field. In this case $K$ is called a contact structure and $\alpha$ is a contact form. Let $\phi: N \longrightarrow N^{\prime}$ be a diffeomorphism between contact manifolds $(N, K)$ and $\left(N^{\prime}, K^{\prime}\right)$. We say that $\phi$ is a contact diffeomorphism if $d \phi(K)=K^{\prime}$. Two contact manifolds $(N, K)$ and $\left(N^{\prime}, K^{\prime}\right)$ are contact diffeomorphic if there exists a contact diffeomorphism $\phi: N \longrightarrow N^{\prime}$. A submanifold $i: L \subset N$ of a contact manifold $(N, K)$ is said to be Legendrian if $\operatorname{dim} L=n$ and $d i_{x}\left(T_{x} L\right) \subset K_{i(x)}$ at any $x \in L$. We say that a smooth fiber bundle $\pi: E \longrightarrow M$ is called a Legendrian fibration if its total space $E$ is furnished with a contact structure and its fibers are Legendrian submanifolds. Let $\pi: E \longrightarrow M$ be a Legendrian fibration. For a Legendrian submanifold $i: L \subset E, \pi \circ i: L \longrightarrow M$ is called a Legendrian map. The image of the Legendrian map $\pi \circ i$ is called a wavefront set of $i$ which is denoted by $W(L)$. For any $z \in E$, it is known that there is a local coordinate system $(x, p, y)=\left(x_{1}, \ldots, x_{m}, p_{1}, \ldots, p_{m}, y\right)$ around $z$ such that $\pi(x, p, y)=(x, y)$ and the contact structure is given by the 1 -form $\alpha=d y-\sum_{i=1}^{m} p_{i} d x_{i}$ (cf. [2] 20.3).

In [25] we have shown the basic duality theorem which is the fundamental tool for the study of spacelike hypersurfaces in Minkowski pseudo-spheres. We consider the following four double fibrations:

(1) (a) $H^{n}(-1) \times S_{1}^{n} \supset \Delta_{1}=\{(\boldsymbol{v}, \boldsymbol{w}) \mid\langle\boldsymbol{v}, \boldsymbol{w}\rangle=0\}$,

(b) $\pi_{11}: \Delta_{1} \longrightarrow H^{n}(-1), \pi_{12}: \Delta_{1} \longrightarrow S_{1}^{n}$,

(c) $\theta_{11}=\langle d \boldsymbol{v}, \boldsymbol{w}\rangle\left|\Delta_{1}, \theta_{12}=\langle\boldsymbol{v}, d \boldsymbol{w}\rangle\right| \Delta_{1}$.

(2) (a) $H^{n}(-1) \times L C^{*} \supset \Delta_{2}=\{(\boldsymbol{v}, \boldsymbol{w}) \mid\langle\boldsymbol{v}, \boldsymbol{w}\rangle=-1\}$,

(b) $\pi_{21}: \Delta_{2} \longrightarrow H^{n}(-1), \pi_{22}: \Delta_{2} \longrightarrow L C^{*}$,

(c) $\theta_{21}=\langle d \boldsymbol{v}, \boldsymbol{w}\rangle\left|\Delta_{2}, \theta_{22}=\langle\boldsymbol{v}, d \boldsymbol{w}\rangle\right| \Delta_{2}$.

(3) (a) $L C^{*} \times S_{1}^{n} \supset \Delta_{3}=\{(\boldsymbol{v}, \boldsymbol{w}) \mid\langle\boldsymbol{v}, \boldsymbol{w}\rangle=1\}$,

(b) $\pi_{31}: \Delta_{3} \longrightarrow L C^{*}, \pi_{32}: \Delta_{3} \longrightarrow S_{1}^{n}$,

(c) $\theta_{31}=\langle d \boldsymbol{v}, \boldsymbol{w}\rangle\left|\Delta_{3}, \theta_{32}=\langle\boldsymbol{v}, d \boldsymbol{w}\rangle\right| \Delta_{3}$.

(4) (a) $L C^{*} \times L C^{*} \supset \Delta_{4}=\{(\boldsymbol{v}, \boldsymbol{w}) \mid\langle\boldsymbol{v}, \boldsymbol{w}\rangle=-2\}$,

(b) $\pi_{41}: \Delta_{4} \longrightarrow L C^{*}, \pi_{42}: \Delta_{4} \longrightarrow L C^{*}$,

(c) $\theta_{41}=\langle d \boldsymbol{v}, \boldsymbol{w}\rangle\left|\Delta_{4}, \theta_{42}=\langle\boldsymbol{v}, d \boldsymbol{w}\rangle\right| \Delta_{4}$.

Here, $\pi_{i 1}(\boldsymbol{v}, \boldsymbol{w})=\boldsymbol{v}, \pi_{i 2}(\boldsymbol{v}, \boldsymbol{w})=\boldsymbol{w},\langle d \boldsymbol{v}, \boldsymbol{w}\rangle=-w_{0} d v_{0}+\sum_{i=1}^{n} w_{i} d v_{i}$ and $\langle\boldsymbol{v}, d \boldsymbol{w}\rangle=$ $-v_{0} d w_{0}+\sum_{i=1}^{n} v_{i} d w_{i}$.

We remark that $\theta_{i 1}^{-1}(0)$ and $\theta_{i 2}^{-1}(0)$ define the same tangent hyperplane field over $\Delta_{i}$ which is denoted by $K_{i}$. The basic duality theorem is the following theorem:

Theorem 3.1. Under the same notations as the previous paragraph, each $\left(\Delta_{i}, K_{i}\right)(i=1,2,3,4)$ is a contact manifold and both of $\pi_{i j}(j=1,2)$ are Legendrian fibrations. Moreover those contact manifolds are contact diffeomorphic to each other.

Since the proof of the theorem was given in [25], we do not give the detailed proof here. We only remark that $\left(\Delta_{1}, K_{1}\right)$ can be canonically identified with the unit tangent bundle 
$S\left(T H^{n}(-1)\right)$ over $H^{n}(-1)$ with the canonical contact structure $([7,9])$. Moreover, the contact structure $K_{i}(i=2,3,4)$ can be canonically induced by the following constructions. We consider smooth mappings $(i \neq j ;(i, j=1,2,3,4)) \Psi_{i j}: \Delta_{i} \longrightarrow \Delta_{j}$ defined by

$$
\begin{aligned}
& \Psi_{12}(\boldsymbol{v}, \boldsymbol{w})=(\boldsymbol{v}, \boldsymbol{v}+\boldsymbol{w}), \Psi_{21}=(\boldsymbol{v}, \boldsymbol{w}-\boldsymbol{v}) \\
& \Psi_{13}(\boldsymbol{v}, \boldsymbol{w})=(\boldsymbol{v}+\boldsymbol{w}, \boldsymbol{w}), \Psi_{31}(\boldsymbol{v}, \boldsymbol{w})=(\boldsymbol{v}-\boldsymbol{w}, \boldsymbol{w}) \\
& \Psi_{14}(\boldsymbol{v}, \boldsymbol{w})=(\boldsymbol{v}-\boldsymbol{w}, \boldsymbol{v}+\boldsymbol{w}), \Psi_{41}(\boldsymbol{v}, \boldsymbol{w})=\left(\frac{\boldsymbol{v}+\boldsymbol{w}}{2}, \frac{\boldsymbol{w}-\boldsymbol{v}}{2}\right) \\
& \Psi_{23}(\boldsymbol{v}, \boldsymbol{w})=(\boldsymbol{w}, \boldsymbol{w}-\boldsymbol{v}), \Psi_{32}(\boldsymbol{v}, \boldsymbol{w})=(\boldsymbol{v}-\boldsymbol{w}, \boldsymbol{v}) \\
& \Psi_{24}(\boldsymbol{v}, \boldsymbol{w})=(2 \boldsymbol{v}-\boldsymbol{w}, \boldsymbol{w}), \Psi_{42}(\boldsymbol{v}, \boldsymbol{w})=\left(\frac{\boldsymbol{v}+\boldsymbol{w}}{2}, \boldsymbol{w}\right) \\
& \Psi_{34}(\boldsymbol{v}, \boldsymbol{w})=(\boldsymbol{v}-2 \boldsymbol{w}, \boldsymbol{v}), \Psi_{43}(\boldsymbol{v}, \boldsymbol{w})=\left(\boldsymbol{w},-\frac{\boldsymbol{v}-\boldsymbol{w}}{2}\right)
\end{aligned}
$$

We can easily show that $\Psi_{i j}$ are contact diffeomorphisms such that $\Psi_{i j}^{-1}=\Psi_{j i}$ for any $i, j=$ $1,2,3,4$. For example, we have

$$
\Psi_{12}^{*} \theta_{21}=\langle d \boldsymbol{v}, \boldsymbol{v}+\boldsymbol{w}\rangle\left|\Delta_{1}=(\langle d \boldsymbol{v}, \boldsymbol{v}\rangle+\langle d \boldsymbol{v}, \boldsymbol{w}\rangle)\right| \Delta_{1}=\langle d \boldsymbol{v}, \boldsymbol{w}\rangle \mid \Delta_{1}=\theta_{11}
$$

and

$$
\begin{aligned}
\Psi_{41}^{*} \theta_{11} & =\left\langle d\left(\frac{\boldsymbol{v}+\boldsymbol{w}}{2}\right), \frac{\boldsymbol{v}-\boldsymbol{w}}{2}\right\rangle \mid \Delta_{4} \\
& =\frac{1}{4}(\langle d \boldsymbol{v}, \boldsymbol{v}\rangle-\langle d \boldsymbol{v}, \boldsymbol{w}\rangle+\langle d \boldsymbol{w}, \boldsymbol{v}\rangle-\langle d \boldsymbol{w}, \boldsymbol{w}\rangle) \mid \Delta_{4} \\
& =\frac{1}{4}(-2\langle d \boldsymbol{v}, \boldsymbol{w}\rangle)\left|\Delta_{4}=-\frac{1}{2}\langle d \boldsymbol{v}, \boldsymbol{w}\rangle\right| \Delta_{4}=-\frac{1}{2} \theta_{41}
\end{aligned}
$$

Therefore $\Psi_{12}:\left(\Delta_{1}, K_{1}\right) \longrightarrow\left(\Delta_{2}, K_{2}\right)$ and $\Psi_{41}:\left(\Delta_{4}, K_{4}\right) \longrightarrow\left(\Delta_{1}, K_{1}\right)$ are contact diffeomorphisms. By the similar calculations, we can show that the other $\Psi_{i j}$ are also contact diffeomorphisms. We call these Legendrian dualities a mandala of Legendrian dualities (cf.,[10, 26]) because we can explain the situation as the following diagram: 


$$
H^{n}(-1) \times S_{1}^{n}
$$

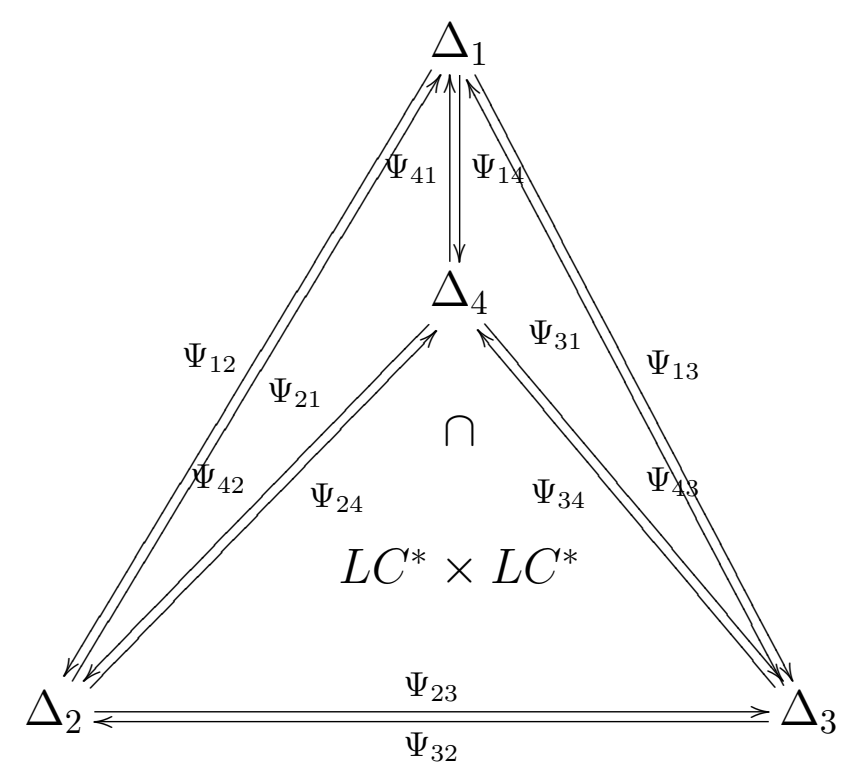

$\cap$

$\cap$

$$
H^{n}(-1) \times L C^{*} \quad L C^{*} \times S_{1}^{n}
$$

The mandala of Legendrian dualities

The above mandala has the similar structure as the real mandala of Buddhism which is a religious picture of the universe. In the real mandala, the central Buddha is the symbol of the sun (the light). In the above diagram the central contact manifold is corresponding to the light, so that the analogous structure exists. This is the reason why we call the above diagram the mandala of Legendrian dualities. The mandala was generalized into the case for pseudo-spheres in general semi-Euclidean space[10]. Moreover, it can be extend to infinitely many Legendrian dualities[26].

\section{Local differential geometry of spacelike hypersurfaces in pseudo-spheres}

In this section we consider differential geometry of hypersurfaces in pseudo-spheres as an application of the mandala of Legendrian dualities. We remark that it is deeply related to the previous theory on the differential geometry of submanifold in the hyperbolic space[17]. We now give a quick review on the theory. Let $\boldsymbol{X}: U \longrightarrow H^{n}(-1)$ be an embedding from an open region $U \subset \mathbb{R}^{n-1}$ and denote that $M=\boldsymbol{X}(U)$. We define the unit normal vector field 
$\boldsymbol{e}: U \longrightarrow S_{1}^{n}$ along $M$ in $H^{n}(-1)$ by

$$
\boldsymbol{e}(u)=\frac{\boldsymbol{X}(u) \wedge \boldsymbol{X}_{u_{1}}(u) \wedge \cdots \wedge \boldsymbol{X}_{u_{n-1}}(u)}{\left\|\boldsymbol{X}(u) \wedge \boldsymbol{X}_{u_{1}}(u) \wedge \cdots \wedge \boldsymbol{X}_{u_{n-1}}(u)\right\|} .
$$

Therefore it satisfies that

$$
\langle\boldsymbol{X}(u), \boldsymbol{e}(u)\rangle=\left\langle\boldsymbol{X}_{u_{i}}(u), \boldsymbol{e}(u)\right\rangle=\left\langle\boldsymbol{X}(u), \boldsymbol{e}_{u_{i}}(u)\right\rangle=0,
$$

where $i=1, \ldots, n-1$ and $\boldsymbol{X}_{u_{i}}=\partial \boldsymbol{X} / \partial u_{i}$. Since $\left\langle\boldsymbol{e}(u), \boldsymbol{e}_{u_{i}}(u)\right\rangle=0$, the above relations mean that $\boldsymbol{e}_{u_{i}}(u)$ is tangent to $M$ at $p=\boldsymbol{X}(u)$. Therefore $d \boldsymbol{e}(u)$ can be considered as a linear transformation on $T_{p} M$. Moreover, if we consider $\mathbb{L}^{ \pm}(u)=\boldsymbol{X}(u) \pm \boldsymbol{e}(u)$, then $\mathbb{L}^{ \pm}(u)$ are lightlike vectors. By the identification of $M$ with $U$ through $\boldsymbol{X}, d \boldsymbol{X}(u)$ can be identified with $1_{T_{p} M}$. Therefore we have a linear transformation $d \mathbb{L}^{ \pm}(u): T_{p} M \longrightarrow T_{p} M$ with $d \mathbb{L}^{ \pm}(u)=1_{T_{p} M} \pm d \boldsymbol{e}(u)$. We call the linear transformation $S_{p}^{ \pm}=-d \mathbb{L}^{ \pm}(u): T_{p} M \longrightarrow T_{p} M$ the hyperbolic shape operator of $M=\boldsymbol{X}(U)$ at $p=\boldsymbol{X}(u)$. The de Sitter Gauss-Kronecker curvature of $M=\boldsymbol{X}(U)$ at $p=\boldsymbol{X}(u)$ is defined to be $K_{d}(u)=\operatorname{det} A_{p}$ and the lightcone Gauss-Kronecker curvature of $M=\boldsymbol{X}(U)$ at $p=\boldsymbol{X}(u)$ is $K_{\ell}^{ \pm}(u)=\operatorname{det} S_{p}^{ \pm}$. In [17] we have investigated the geometric meanings of the lightcone Gauss-Kronecker curvature from the contact viewpoint. One of the consequences is that the lightcone Gauss-Kronecker curvature estimates the contact of hypersurfaces with hyperhorospheres. It has been also shown that the Gauss-Bonnet type theorem holds on the lightcone Gauss-Kronecker curvature [25].

On the other hand, we can interpret the above construction by using the Legendrian duality theorem (Theorem 3.1). For any regular hypersurface $\boldsymbol{X}: U \longrightarrow H^{n}(-1)$, we have $\left\langle\boldsymbol{X}(u), \mathbb{L}^{ \pm}(u)\right\rangle=-1$. Therefore, we can define an embeddings $\mathcal{L}_{2}^{ \pm}: U \longrightarrow \Delta_{2}$ by $\mathcal{L}_{2}^{ \pm}(u)=$ $\left(\boldsymbol{X}(u), \mathbb{L}^{ \pm}(u)\right)$. Since $\left\langle\boldsymbol{X}_{u_{i}}(u), \mathbb{L}^{ \pm}(u)\right\rangle=0$, each of $\mathcal{L}_{2}^{ \pm}$are Legendrian embeddings.

It has been shown that $\pi_{21}: \Delta_{2} \longrightarrow H^{n}(-1)$ is a Legendrian fibration. The fiber is the intersection of $L C^{*}$ with a spacelike hyperplane (i.e., an elliptic hyperquadric). Therefore the intersection of the fiber with the normal plane (i.e., a timelike plane) in $\mathbb{R}_{1}^{n+1}$ of $M$ consists of two points at each point of $M$. This is the reason why we have such two Legendrian embeddings. However, one of the results in the theory of Legendrian singularities (cf., the appendix) asserts that the Legendrian submanifold is uniquely determined by the wave front set at least locally. Here, $M=\boldsymbol{X}(U)=\pi_{21} \circ \mathcal{L}_{4}^{ \pm}(U)$ is the wave front set of $\mathcal{L}_{2}^{ \pm}(U)$ through the Legendrian fibration $\pi_{21}$. Therefore each of the Legendrian embeddings $\mathcal{L}_{2}^{ \pm}$is uniquely determined with respect to $M=\boldsymbol{X}(U)$. It follows that we have a unique pair of lightcone Gauss images $\mathbb{L}^{ \pm}=\pi_{22} \circ \mathcal{L}_{2}^{ \pm}$. Moreover, we have a Legendrian embedding $\mathcal{L}_{1}: U \longrightarrow \Delta_{1}$ defined by $\mathcal{L}_{1}(u)=(\boldsymbol{X}(u), \boldsymbol{e}(u))$. It follows from the mandala of Legendrian dualities, we have

$$
\mathcal{L}_{3}(u)=\Psi_{13} \circ \mathcal{L}_{1}(u)=\left(\mathbb{L}^{+}(u), \boldsymbol{e}(u)\right), \mathcal{L}_{4}(u)=\Psi_{14} \circ \mathcal{L}_{1}(u)=\left(\mathbb{L}^{-}(u), \mathbb{L}^{+}(u)\right) .
$$

We write $\mathcal{L}_{2}(u)=\mathcal{L}_{2}^{+}(u)$. Eventually we have Legendrian embeddings $\mathcal{L}_{i}: U \longrightarrow \Delta_{i}(i=$ $1,2,3,4)$ such that $\Psi_{i j} \circ \mathcal{L}_{i}=\mathcal{L}_{j}$. In this case we started the embedding $\boldsymbol{X}: U \longrightarrow H^{n}(-1)$. However, we have no reason why we do not start a spacelike embedding into $S_{1}^{n}$ or $L C^{*}$.

According to the above arguments, we consider the following situations. Let $\mathcal{L}_{1}: U \longrightarrow \Delta_{1}$ be a Legendrian embedding and denote that $\mathcal{L}_{1}(u)=\left(\boldsymbol{X}^{h}(u), \boldsymbol{X}^{d}(u)\right)$. By using the contact diffeomorphism $\Psi_{14}$, we have a Legendrian embedding $\mathcal{L}_{4}: U \longrightarrow \Delta_{4}$ defined by $\mathcal{L}_{4}(u)=$ $\Psi_{14} \circ \mathcal{L}_{1}(u)$. We denote that $\mathcal{L}_{4}(u)=\left(\boldsymbol{X}_{-}^{\ell}(u), \boldsymbol{X}_{+}^{\ell}(u)\right)$, so that we have the following relations:

$$
\begin{gathered}
\boldsymbol{X}_{-}^{\ell}(u)=\boldsymbol{X}^{h}(u)-\boldsymbol{X}^{d}(u), \boldsymbol{X}_{+}^{\ell}(u)=\boldsymbol{X}^{h}(u)+\boldsymbol{X}^{d}(u), \\
\boldsymbol{X}^{h}(u)=\frac{\boldsymbol{X}_{+}^{\ell}(u)+\boldsymbol{X}_{-}^{\ell}(u)}{2}, \boldsymbol{X}^{d}(u)=\frac{\boldsymbol{X}_{+}^{\ell}(u)-\boldsymbol{X}_{-}^{\ell}(u)}{2} .
\end{gathered}
$$


We also denote that $\mathcal{L}_{2}=\Psi_{12} \circ \mathcal{L}_{1}: U \longrightarrow \Delta_{2}$ and $\mathcal{L}_{3}=\Psi_{13} \circ \mathcal{L}_{1}: U \longrightarrow \Delta_{3}$, so that we have

$$
\mathcal{L}_{2}(u)=\left(\boldsymbol{X}^{h}(u), \boldsymbol{X}_{+}^{\ell}(u)\right), \mathcal{L}_{3}(u)=\left(\boldsymbol{X}_{+}^{\ell}(u), \boldsymbol{X}^{d}(u)\right) .
$$

Since $\Psi_{i j}(i, j=1,2,3,4)$ are contact diffeomorphisms, $\mathcal{L}_{i}(U)(i=1,2,3,4)$ are Legendrian submanifolds. By defintion, $\mathcal{L}_{1}(U)$ is a Legendrian submanifold in $\Delta_{1}$ if and only if

$$
\left\langle\boldsymbol{X}^{h}(u), \boldsymbol{X}^{d}(u)\right\rangle=\left\langle\boldsymbol{X}^{h}(u), \boldsymbol{X}_{u_{i}}^{d}(u)\right\rangle=\left\langle\boldsymbol{X}_{u_{i}}^{h}(u), \boldsymbol{X}^{d}(u)\right\rangle=0
$$

for $i=1, \ldots, n-1$. Therefore if we suppose that $\boldsymbol{X}^{h}$ is an embedding, then $\boldsymbol{X}^{d}$ can be considered as the Gauss map of $M^{h}=\boldsymbol{X}^{h}(U)$ and $-d \boldsymbol{X}^{d}(u)$ is the corresponding Weingarten map. If $\boldsymbol{X}^{d}$ is an embedding, then $\boldsymbol{X}^{h}$ can be considered as the Gauss map of $M^{d}=\boldsymbol{X}^{d}(U)$ and $-d \boldsymbol{X}^{h}(u)$ is the corresponding Weingarten map. It follows that we can define the corresponding curvatures. The situations are the same as for the other $\mathcal{L}_{i}(U)$. We now summarize the situations. We denote that $M^{H}=\boldsymbol{X}^{h}(U)$ and $M^{D}=\boldsymbol{X}^{d}(U)$. If $\boldsymbol{X}^{h}$ is an embedding, we call $\boldsymbol{X}^{d}$ the de Sitter Gauss image of hypersurface $M^{H}$ in the hyperbolic space $H^{n}(-1)$. Moreover, we define $\left(S_{d}^{H}\right)_{p}=-d \boldsymbol{X}^{d}(u): T_{p} M^{H} \longrightarrow T_{p} M^{H}$ where $p=\boldsymbol{X}^{h}(u)$. We also call $\left(S_{d}^{H}\right)_{p}$ the de Sitter Weingarten map of hypersurface $M^{H}$ in the hyperbolic space $H^{n}(-1)$ at $p=\boldsymbol{X}^{h}(u)$. Then we have de Sitter principal curvatures $\kappa_{d, i}^{H}(u)(i=1, \ldots, n-1)$ defined as the eigenvalues of $\left(S_{d}^{H}\right)_{p}$ and the de Sitter Gauss-Kronecker curvature $K_{d}^{H}(u)=\operatorname{det}\left(S_{d}^{H}\right)_{p}$ of $M^{H}$ at $p=\boldsymbol{X}^{h}(u)$.

On the other hand, if $\boldsymbol{X}^{d}$ is an embedding, we call $\boldsymbol{X}^{h}$ the hyperbolic Gauss image of spacelike hypersurface $M^{D}$ in the d Sitter space $S_{1}^{n}$. Moreover, we define $\left(S_{h}^{D}\right)_{p}=-d \boldsymbol{X}^{h}(u)$ : $T_{p} M^{D} \longrightarrow T_{p} M^{D}$ where $p=\boldsymbol{X}^{d}(u)$. We also call $\left(S_{h}^{D}\right)_{p}$ the hyperbolic Weingarten map of spacelike hypersurface $M^{D}$ in the de Sitter space $S_{1}^{n}$ at $p=\boldsymbol{X}^{d}(u)$. Then we have hyperbolic principal curvatures $\kappa_{h, i}^{D}(u)(i=1, \ldots, n-1)$ defined as the eigenvalues of $\left(S_{h}^{D}\right)_{p}$ and the hyperbolic Gauss-Kronecker curvature $K_{h}^{D}(u)=\operatorname{det}\left(S_{h}^{D}\right)_{p}$ of $M^{D}$ at $p=\boldsymbol{X}^{d}(u)$. If both the mappings $\boldsymbol{X}^{h}, \boldsymbol{X}^{d}$ are embeddings, then we define $g_{i j}^{D}(u)=\left\langle\boldsymbol{X}_{i}^{h}(u), \boldsymbol{X}_{j}^{h}(u)\right\rangle, g_{i j}^{H}(u)=\left\langle\boldsymbol{X}_{i}^{d}(u), \boldsymbol{X}_{j}^{d}(u)\right\rangle$ and $h_{i j}^{\Delta_{1}}(u)=-\left\langle\boldsymbol{X}_{i}^{d}(u), \boldsymbol{X}_{j}^{h}(u)\right\rangle=\left\langle\boldsymbol{X}_{i j}^{d}(u), \boldsymbol{X}^{h}(u)\right\rangle=\left\langle\boldsymbol{X}^{d}(u), \boldsymbol{X}_{i j}^{h}(u)\right\rangle$. We respectively call $g_{i j}^{H}, g_{i j}^{D}$ and $h_{i j}^{\Delta_{1}}$ a hyperbolic first fundamental invariant of $M^{D}$, a de Sitter first fundamental invariant of $M^{H}$ and a $\Delta_{1}$-second fundamental invariant. In this case we can identify $T_{p} M^{H}$ with $T_{p}^{\prime} M^{D}$ for $p=\boldsymbol{X}^{h}(u)$ and $p^{\prime}=\boldsymbol{X}^{d}(u)$. By definition, the principal directions of $\left(S_{d}^{H}\right)_{p}$ and $\left(S_{h}^{D}\right)_{p}^{\prime}$ are the same. We have the following Weingarten type formulae.

Proposition 4.1. Let $\mathcal{L}_{1}: U \longrightarrow \Delta_{1}$ be a Legedrian embedding with $\mathcal{L}_{1}(u)=\left(\boldsymbol{X}^{h}(u), \boldsymbol{X}^{d}(u)\right)$. (1) Suppose that $\boldsymbol{X}^{h}: U \longrightarrow H^{n}(-1)$ is an embedding. Then we have

$$
\boldsymbol{X}_{u_{i}}^{d}=-\sum_{j=1}^{n-1}\left(h^{D}\right)_{i}^{j} \boldsymbol{X}_{u_{j}}^{h} .
$$

(2) Suppose that $\boldsymbol{X}^{d}: U \longrightarrow H^{n}(-1)$ is an embedding. Then we have

$$
\boldsymbol{X}_{u_{i}}^{h}=-\sum_{j=1}^{n-1}\left(h^{H}\right)_{i}^{j} \boldsymbol{X}_{u_{j}}^{d} .
$$

Here, $\left(\left(h^{H}\right)_{i}^{j}\right)=\left(h_{i j}^{\Delta_{1}}\right)\left(g_{i j}^{H}\right)^{-1}$ and $\left(\left(h^{D}\right)_{i}^{j}\right)=\left(h_{i j}^{\Delta_{1}}\right)\left(g_{i j}^{D}\right)^{-1}$.

The proof of the above formulae are the same as those for the Weingarten type formula in [17], so that we omit it. We remark that $\kappa_{d, i}^{H}(u)$ and $\kappa_{h, i}^{D}(u)$ are the eigenvalues of $\left(\left(h^{H}\right)_{i}^{j}\right)$ and $\left(\left(h^{D}\right)_{i}^{j}\right)$ respectively. We have the following relation between $\kappa_{d, i}^{H}(u)$ and $\kappa_{h, i}^{D}(u)$. 
Corollary 4.2. Suppose that both the mappings $\boldsymbol{X}^{h}, \boldsymbol{X}^{d}$ are embeddings. In this case we have the relation $\kappa_{d, i}^{H}(u) \kappa_{h, i}^{D}(u)=1(i=1, \ldots n-1)$. Here $\kappa_{d, i}^{H}(u)$ and $\kappa_{h, i}^{D}(u)$ are principal curvatures corresponding to the same principal direction.

Proof. Since both the mappings $\boldsymbol{X}^{h}, \boldsymbol{X}^{d}$ are embeddings, $K_{d}^{H}(u) \neq 0$ and $K_{h}^{D}(u) \neq 0$. By the Weingarten type formulae, $\left(\left(h^{D}\right)_{i}^{j}\right)$ is the inverse matrix of $\left(\left(h^{H}\right)_{i}^{j}\right)$, so that the eigenvalues have the above relations.

We say that $\pi_{i 1} \circ \mathcal{L}_{i}$ and $\pi_{i 2} \circ \mathcal{L}_{i}$ are $\Delta_{i}$-dual each other if $\mathcal{L}_{i}: U \longrightarrow \Delta_{i}$ is an isotropic mapping with respect to $K_{i}$.

\section{$5 \quad$ Linear Weingarten surfaces}

Galvez, Martinez and Milan has investigated the linear Weingarten surfaces using the Weierstrass type representation formula [12]. In this section, we discuss linear Weingarten surfaces and their hyperbolic Gauss maps from our point of view.

\subsection{Matrix representation of pseudo-spheres}

Let $\operatorname{Herm}(2)$ be the set of $2 \times 2$ Hermitian matrices. Identifying via

$$
\mathbb{R}_{1}^{4} \ni\left(x_{0}, x_{1}, x_{2}, x_{3}\right) \mapsto \sum_{k=0}^{3} x_{k} \boldsymbol{e}_{k}=\left(\begin{array}{cc}
x_{0}+x_{3} & x_{1}+\sqrt{-1} x_{2} \\
x_{1}-\sqrt{-1} x_{2} & x_{0}-x_{3}
\end{array}\right) \in \operatorname{Herm}(2)
$$

where

$$
\boldsymbol{e}_{0}=\left(\begin{array}{ll}
1 & 0 \\
0 & 1
\end{array}\right), \boldsymbol{e}_{1}=\left(\begin{array}{ll}
0 & 1 \\
1 & 0
\end{array}\right), \boldsymbol{e}_{2}=\left(\begin{array}{cc}
0 & \sqrt{-1} \\
-\sqrt{-1} & 0
\end{array}\right), \boldsymbol{e}_{3}=\left(\begin{array}{cc}
1 & 0 \\
0 & -1
\end{array}\right)
$$

one has $\langle\boldsymbol{x}, \boldsymbol{y}\rangle=\operatorname{trace}\left(\boldsymbol{x} \boldsymbol{e}_{2}\left({ }^{t} \boldsymbol{y}\right) \boldsymbol{e}_{2}\right)=\operatorname{trace}(\boldsymbol{x} \tilde{\boldsymbol{y}})$, where $\tilde{\boldsymbol{y}}$ is the cofactor matrix of $\boldsymbol{y}$. In particular, we have $\langle\boldsymbol{x}, \boldsymbol{x}\rangle=-\operatorname{det} \boldsymbol{x}$. Hence the pseudo-spheres are represented as

$$
\begin{aligned}
H_{+}^{3}(-1) & =\{X \in \operatorname{Herm}(2) \mid \operatorname{det} X=1, \operatorname{trace} X>0\}=\left\{A A^{*} \mid A \in S L(2, \boldsymbol{C})\right\} \\
S_{1}^{3} & =\{X \in \operatorname{Herm}(2) \mid \operatorname{det} X=-1\}=\left\{A \boldsymbol{e}_{3} A^{*} \mid A \in S L(2, \boldsymbol{C})\right\} \\
L C^{*} & =\{X \in \operatorname{Herm}(2) \mid \operatorname{det} X=0, \operatorname{trace} X>0\} \\
& =\left\{A \boldsymbol{m}_{1} A^{*} \mid A \in S L(2, \boldsymbol{C})\right\}=\left\{A \boldsymbol{m}_{2} A^{*} \mid A \in S L(2, \boldsymbol{C})\right\}
\end{aligned}
$$

where

$$
\boldsymbol{m}_{1}=\boldsymbol{e}_{0}+\boldsymbol{e}_{3}=\left(\begin{array}{ll}
2 & 0 \\
0 & 0
\end{array}\right), \quad \boldsymbol{m}_{2}=\boldsymbol{e}_{0}-\boldsymbol{e}_{3}=\left(\begin{array}{ll}
0 & 0 \\
0 & 2
\end{array}\right) .
$$

If we define an action of $g \in S L(2, \boldsymbol{C})$ to $\boldsymbol{x} \in \mathbb{R}_{1}^{4}$ by $g \cdot \boldsymbol{x}=g \boldsymbol{x} g^{*}$, then the above pseudo-spheres are invariant under this action, thus we have the another representation:

$$
H_{+}^{3}(-1)=\left\{A \boldsymbol{x} A^{*} \mid A \in S L(2, \boldsymbol{C}), \boldsymbol{x} \in \operatorname{Herm}(2), \operatorname{det} \boldsymbol{x}=1\right\} .
$$

\subsection{Dualities of surfaces in pseudo-spheres}

In the context of our duality, as in Section 4, the following lemma holds. 
Lemma 5.1. Let $\boldsymbol{X}^{h}: U^{2} \longrightarrow H_{+}^{3}(-1)$ be a surface and $\boldsymbol{X}^{d}$ the hyperbolic Gauss map of $\boldsymbol{X}^{h}$. Then

$$
\boldsymbol{X}^{h} \in H_{+}^{3}(-1), \boldsymbol{X}^{d} \in S_{1}^{3}, \boldsymbol{X}^{h}+\boldsymbol{X}^{d} \in L C^{*}, \boldsymbol{X}^{h}-\boldsymbol{X}^{d} \in L C^{*}
$$

are dual each other in the sense of Theorem 3.1.

Proof. This lemma is a conclusion from the general theory of duality as in Section 4. But we give an alternative proof here. It is based on a direct calculation. By the definition, we have $\left\langle\boldsymbol{X}^{h}, \boldsymbol{X}^{d}\right\rangle=0$ and $\left\langle d \boldsymbol{X}^{h}, \boldsymbol{X}^{d}\right\rangle=0$. Hence $\boldsymbol{X}^{h}$ and $\boldsymbol{X}^{d}$ are dual. The other cases are obvious because of the definition of dualities [25] and formulas $\left\langle\boldsymbol{X}^{h}, \boldsymbol{X}^{h}+\boldsymbol{X}^{d}\right\rangle=-1$, $\left\langle\boldsymbol{X}^{d}, \boldsymbol{X}^{h}+\boldsymbol{X}^{d}\right\rangle=\left\langle-\boldsymbol{X}^{d}, \boldsymbol{X}^{h}-\boldsymbol{X}^{d}\right\rangle=1$ and $\left\langle\boldsymbol{X}^{h}+\boldsymbol{X}^{d}, \boldsymbol{X}^{h}-\boldsymbol{X}^{d}\right\rangle=-2$.

\subsection{Linear Weingarten surfaces of Bryant type and Bianchi type}

A surface $f: U^{2} \rightarrow H_{+}^{3}(-1)$ is called a linear Weingarten surface if the mean curvature $H_{d}^{H}=\left(\kappa_{1}^{H}+\kappa_{2}^{H}\right) / 2$ and the de Sitter Gauss-Kronecker curvature $K_{d}^{H}$ satisfies

$$
2 a\left(H_{d}^{H}-1\right)+b\left(K_{d}^{H}-1\right)=0, \quad a, b \in \mathbb{R}, \quad a+b \neq 0 .
$$

If $a+b \neq 0$ holds, it is called a linear Weingarten surface of Bryant type. In [22], we investigated "horo-flat" horospherical surfaces in $H_{+}^{3}(-1)$. It is linear Weingarten surfaces of non-Bryant type, we considered them as surfaces whose hyperbolic Gauss map degenerates to a curve in the de Sitter space (see [22, Section 4]). This means that a horo-flat horospherical surface is the dual surface of a curve in the de Sitter space. In [12], Galvez, Martinez and Milan showed the following representation formula for linear Weingarten surfaces of Bryant type.

Theorem 5.2. [12, Theorem 2] Let $V \subset \boldsymbol{C}$ be a simply connected domain. Fix a meromorphic map $A: V \longrightarrow S L(2, \boldsymbol{C})$ satisfying

$$
A^{-1} d A=\left(\begin{array}{cc}
0 & \omega \\
d h & 0
\end{array}\right)
$$

where $h$ is a meromorphic function and $\omega$ a holomorphic one-form. If

$$
\sigma=(a+b)\left(\left(1+\varepsilon|h|^{2}\right)^{2}|\omega|^{2}-\frac{(1-\varepsilon)^{2}|d h|^{2}}{\left(1+\varepsilon|h|^{2}\right)^{2}}\right)
$$

is positive definite then $f=A\left(\Omega_{+}\right) A^{*}$ is a linear Weingarten surface. Moreover, the hyperbolic Gauss map $\nu$ of $f$ is given by $\nu=A\left(\Omega_{-}\right) A^{*}$ where

$$
\Omega_{ \pm}=\left(\begin{array}{cc}
\frac{1 \pm \varepsilon^{2}|h|^{2}}{1+\varepsilon|h|^{2}} & \mp \varepsilon \bar{h} \\
\mp \varepsilon h & \pm\left(1+\varepsilon|h|^{2}\right)
\end{array}\right), \text { respectively, } \quad \varepsilon=a /(a+b), \quad \text { and } \quad 1+\varepsilon|h|^{2}>0 .
$$

By Lemma 5.1, we can obtain the dual surfaces in $S_{1}^{3}$ and $L C^{*}$ by taking $\nu: U^{2} \rightarrow S_{1}^{3}$ and $f \pm \nu: U^{2} \rightarrow L C^{*}:$

$$
f+\nu=A\left(\begin{array}{cc}
2 \frac{1}{1+\varepsilon|h|^{2}} & 0 \\
0 & 0
\end{array}\right) A^{*}, \quad f-\nu=A\left(\begin{array}{cc}
2 \frac{\varepsilon^{2}|h|^{2}}{1+\varepsilon|h|^{2}} & -2 \varepsilon \bar{h} \\
-2 \varepsilon h & 2\left(1+\varepsilon|h|^{2}\right)
\end{array}\right) A^{*} .
$$


In [3], Aledo and Espinar showed a Weierstrass type representation formula for linear Weingarten surfaces of Bianchi type. A surface $f: U^{2} \rightarrow S_{1}^{3}$ is a linear Weingarten surface if the mean curvature $H_{h}^{D}$ and the hyperbolic Gauss-Kronecker curvature $K_{h}^{D}$ satisfies

$$
2 A\left(H_{h}^{D}-1\right)+B\left(K_{h}^{D}-1\right)=0, \quad A, B \in \mathbb{R} .
$$

If $A+B \neq 0$ holds, it is called Bianchi type. As a consequence of the duality theorem, we can interpret the relationship between linear Weingarten surfaces in $H_{+}^{3}(-1)$ and $S_{1}^{3}$.

Theorem 5.3. Let $\mathcal{L}_{1}: U^{2} \rightarrow \Delta_{1}$ be a Legendrian immersion. Suppose that both of $\pi_{11} \circ \mathcal{L}_{1}$ : $U^{2} \rightarrow H_{+}^{3}(-1)$ and $\pi_{12} \circ \mathcal{L}_{1}: U^{2} \rightarrow S_{1}^{3}$ are immersions. Then $\pi_{11} \circ \mathcal{L}_{1}=\boldsymbol{X}^{h}$ is a linear Weingarten surface of Bryant type if and only if $\pi_{12} \circ \mathcal{L}_{1}=\boldsymbol{X}^{d}$ is a linear Weingarten surface of Bianchi type.

Proof. Let $\kappa_{d, i}^{H}(i=1,2)$ be the de Sitter principal curvatures of $M^{H}=\boldsymbol{X}^{h}(U)$ at $p=\boldsymbol{X}^{h}(u)$. and $\kappa_{h, i}^{D}(i=1,2)$ the hyperbolic principal curvatures of $M^{D}=\boldsymbol{X}^{d}(U)$ at $p^{\prime}=\boldsymbol{X}^{d}(u)$. By 4.2, we have the relations $\kappa_{d, i}^{H} \kappa_{h, i}^{D}=1$. Since $K_{d}^{H}(u)=\kappa_{d, 1}^{H} \kappa_{d, 2}^{H}$ and $2 H_{d}^{H}=\kappa_{d, 1}^{H}+\kappa_{d, 2}^{H}$, we have

$$
2 a\left(H_{d}^{H}-1\right)+b\left(K_{d}^{H}-1\right)=a\left(\kappa_{d, 1}^{H}+\kappa_{d, 2}^{H}-2\right)+b\left(\kappa_{d, 1}^{H} \kappa_{d, 2}^{H}-1\right) .
$$

We also have another relation

$$
2 A\left(H_{h}^{D}-1\right)+B\left(K_{d}^{D}-1\right)=A\left(\kappa_{h, 1}^{D}+\kappa_{h, 2}^{D}-2\right)+B\left(\kappa_{h, 1}^{D} \kappa_{h, 2}^{D}-1\right) .
$$

Since $\kappa_{d, i}^{H}=1 / \kappa_{h, i}^{D}$, we have

$$
\begin{aligned}
2 a\left(H_{d}^{H}-1\right)+b\left(K_{d}^{H}-1\right) & =a\left(\frac{1}{\kappa_{h, 1}^{D}}+\frac{1}{\kappa_{h, 2}^{D}}-2\right)+b\left(\frac{1}{\kappa_{h, 1}^{D} \kappa_{h, 2}^{D}}-1\right) \\
& =\frac{1}{\kappa_{h, 1}^{D} \kappa_{h, 2}^{D}}\left(a\left(\kappa_{h, 1}^{D}+\kappa_{h, 2}^{D}-2\right)+(-2 a-b)\left(\kappa_{h, 1}^{D} \kappa_{h, 2}^{D}-1\right)\right. \\
& =\frac{1}{\kappa_{h, 1}^{D} \kappa_{h, 2}^{D}}\left(2 a\left(H_{h}^{D}-1\right)+(-2 a-b)\left(K_{h}^{D}-1\right)\right) .
\end{aligned}
$$

If we put $A=a, B=-(2 a+b)$, then $2 a\left(H_{d}^{H}-1\right)+b\left(K_{d}^{H}-1\right)=0$ if and only if $2 A\left(H_{h}^{D}-1\right)+$ $B\left(K_{h}^{D}-1\right)=0$. Moreover, $A+B=0$ if and only if $a+b=0$. This completes the proof.

This theorem shows that we can bring the representation formula for a surface in $H_{+}^{3}(-1)$ to representation formulas for surfaces in $S_{1}^{3}$ and $L C^{*}$, and get new surfaces. Remark that we have interesting families of surfaces in the lightcone obtained by taking the dual of linear Weingarten surface of non-Bryant type. In fact, the Gauss map $\nu$ of a linear Weingarten surface $f$ given in Theorem 5.2 is a linear Weingarten surface in $S_{1}^{3}$. Furthermore, surfaces $f \pm \nu$ given in (5.1) are this class of surfaces. Theorem 5.3 says that Theorem 5.2 also can be considered representation formula for these families of surfaces. Kokubu and Umehara investigated the topological properities of linear Weingarten surfaces giving a variant of this representation formula [27]. 


\section{The Legendrian dualities for "flat"spacelike surfaces}

In this section we study general properties of spacelike surfaces in pseudo-spheres which are $\Delta_{i}$-duals of spacelike curves in pseudo-spheres. Let $\boldsymbol{a}_{0}: I \longrightarrow H_{+}^{3}(-1)$ be a smooth map and $\boldsymbol{a}_{i}: I \longrightarrow S_{1}^{3}(i=1,2)$ be smooth mappings from an open interval $I$ with $\left\langle\boldsymbol{a}_{i}(t), \boldsymbol{a}_{j}(t)\right\rangle=0$ if $i \neq j$. We define a unit spacelike vector $\boldsymbol{a}_{3}(t)=\boldsymbol{a}_{0}(t) \wedge \boldsymbol{a}_{1}(t) \wedge \boldsymbol{a}_{2}(t)$, so that we have a pseudo-orthonormal frame $\left\{\boldsymbol{a}_{0}, \boldsymbol{a}_{1}, \boldsymbol{a}_{2}, \boldsymbol{a}_{3}\right\}$ of $\mathbb{R}_{1}^{4}$. We have the following fundamental invariants:

$$
\begin{array}{ll}
c_{1}(t)=\left\langle\boldsymbol{a}_{0}^{\prime}(t), \boldsymbol{a}_{1}(t)\right\rangle=-\left\langle\boldsymbol{a}_{0}(t), \boldsymbol{a}_{1}^{\prime}(t)\right\rangle, & c_{4}(t)=\left\langle\boldsymbol{a}_{1}^{\prime}(t), \boldsymbol{a}_{2}(t)\right\rangle=-\left\langle\boldsymbol{a}_{1}(t), \boldsymbol{a}_{2}^{\prime}(t)\right\rangle, \\
c_{2}(t)=\left\langle\boldsymbol{a}_{0}^{\prime}(t), \boldsymbol{a}_{2}(t)\right\rangle=-\left\langle\boldsymbol{a}_{0}(t), \boldsymbol{a}_{2}^{\prime}(t)\right\rangle, & c_{5}(t)=\left\langle\boldsymbol{a}_{1}^{\prime}(t), \boldsymbol{a}_{3}(t)\right\rangle=-\left\langle\boldsymbol{a}_{1}(t), \boldsymbol{a}_{3}^{\prime}(t)\right\rangle, \\
c_{3}(t)=\left\langle\boldsymbol{a}_{0}^{\prime}(t), \boldsymbol{a}_{3}(t)\right\rangle=-\left\langle\boldsymbol{a}_{0}(t), \boldsymbol{a}_{3}^{\prime}(t)\right\rangle, & c_{6}(t)=\left\langle\boldsymbol{a}_{2}^{\prime}(t), \boldsymbol{a}_{3}(t)\right\rangle=-\left\langle\boldsymbol{a}_{2}(t), \boldsymbol{a}_{3}^{\prime}(t)\right\rangle .
\end{array}
$$

It can be written in the following form:

$$
\left(\begin{array}{l}
\boldsymbol{a}_{0}^{\prime}(t) \\
\boldsymbol{a}_{1}^{\prime}(t) \\
\boldsymbol{a}_{2}^{\prime}(t) \\
\boldsymbol{a}_{3}^{\prime}(t)
\end{array}\right)=\left(\begin{array}{cccc}
0 & c_{1}(t) & c_{2}(t) & c_{3}(t) \\
c_{1}(t) & 0 & c_{4}(t) & c_{5}(t) \\
c_{2}(t) & -c_{4}(t) & 0 & c_{6}(t) \\
c_{3}(t) & -c_{5}(t) & -c_{6}(t) & 0
\end{array}\right)\left(\begin{array}{l}
\boldsymbol{a}_{0}(t) \\
\boldsymbol{a}_{1}(t) \\
\boldsymbol{a}_{2}(t) \\
\boldsymbol{a}_{3}(t)
\end{array}\right)=: C(t)\left(\begin{array}{l}
\boldsymbol{a}_{0}(t) \\
\boldsymbol{a}_{1}(t) \\
\boldsymbol{a}_{2}(t) \\
\boldsymbol{a}_{3}(t)
\end{array}\right)
$$

We remark that $C(t)$ is an element of the Lie algebra $\mathfrak{s o}(3,1)$ of the Lorentzian group $S O_{0}(3,1)$. If $\left\{\boldsymbol{a}_{0}(t), \boldsymbol{a}_{1}(t), \boldsymbol{a}_{2}(t), \boldsymbol{a}_{3}(t)\right\}$ is a pseudo-orthonormal frame field as the above, the $4 \times 4$-matrix determined by the frame defines a smooth curve $A: I \longrightarrow S O_{0}(3,1)$. Therefore we have the relation that $A^{\prime}(t)=C(t) A(t)$. For the converse, let $A: I \longrightarrow S O_{0}(3,1)$ be a smooth curve, then we can show that $A^{\prime}(t) A(t)^{-1} \in \mathfrak{s o}(3,1)$. Moreover, for any smooth curve $C: I \longrightarrow \mathfrak{s o}(3,1)$, we apply the existence theorem on the linear systems of ordinary differential equations, so that there exists a unique curve $A: I \longrightarrow S O_{0}(3,1)$ such that $C(t)=A^{\prime}(t) A(t)^{-1}$ with an initial data $A\left(t_{0}\right) \in S O_{0}(3,1)$. Therefore, a smooth curve $C: I \longrightarrow \mathfrak{s o}(3,1)$ might be identified with a pseudo-orthonormal frame in $H_{+}^{3}(-1)$. Let $C: I \longrightarrow \mathfrak{s o}(3,1)$ be a smooth curve with $C(t)=A^{\prime}(t) A(t)^{-1}$ and $B \in S O_{0}(3,1)$, then we have $C(t)=(A(t) B)^{\prime}(A(t) B)^{-1}$. This means that the curve $C: I \longrightarrow \mathfrak{s o}(3,1)$ is a hyperbolic invariant of the pseudo-orthonormal frame $\left\{\boldsymbol{a}_{0}(t), \boldsymbol{a}_{1}(t), \boldsymbol{a}_{2}(t), \boldsymbol{a}_{3}(t)\right\}$. We set $\boldsymbol{\ell}=\boldsymbol{a}_{0}+\boldsymbol{a}_{2}$ and $c_{36}=c_{3}+c_{6}$. We study dual surfaces of $\ell$ using $\Delta_{2}, \Delta_{3}$ and $\Delta_{4}$-duality. In this section, we assume $c_{2}(t)=0, c_{1}(t)-c_{4}(t)=0$ and $c_{36}(t) \neq 0$ for any $t$, this means that $\ell^{\prime}=c_{36} \boldsymbol{a}_{3} \neq 0$.

\section{1 $\Delta_{2}, \Delta_{3}$ and $\Delta_{4}$-dual surfaces of $\ell$}

(1) $\Delta_{2}$-dual surface of $\ell$ : In order to obtain the $\Delta_{2}$-dual surface of $\ell$, we consider a hight function $F: H_{+}^{3}(-1) \times I \longrightarrow \mathbb{R}$ defined by $F(X, t)=\langle X, \ell(t)\rangle+1$. There exist $x_{0}, x_{1}, x_{2}, x_{3} \in \mathbb{R}$ such that $X=x_{0} \boldsymbol{a}_{0}+x_{1} \boldsymbol{a}_{1}+x_{2} \boldsymbol{a}_{2}+x_{3} \boldsymbol{a}_{3}$. Then the discriminant set $D_{F}$ of $F$ is

$$
\begin{aligned}
D_{F} & =\left\{X \in H_{+}^{3}(-1) \mid{ }^{\exists} t \in I, F(X, t)=\frac{\partial F}{\partial t}(X, t)=0\right\} \\
& =\left\{X \in H_{+}^{3}(-1) \mid{ }^{\exists} t \in I,-x_{0}+x_{2}+1=0, x_{3}=0\right\} .
\end{aligned}
$$

Since $X \in H_{+}^{3}(-1)$, we have

$$
X=\boldsymbol{a}_{0}(t)+s \boldsymbol{a}_{1}(t)+\frac{s^{2}}{2} \boldsymbol{\ell}(t)
$$

for $x_{1}=s$, which we denote $\boldsymbol{X}_{\ell}^{h}(s, t)$. 
By the above construction, $\left(\boldsymbol{X}_{\ell}^{h}(s, t), \boldsymbol{\ell}(t)\right): \mathbb{R}^{2} \rightarrow \Delta_{2}$ is an isotropic map with respect to the contact structure defined in Theorem 3.1, so that $\boldsymbol{X}_{\ell}^{h}(s, t)$ and $\boldsymbol{\ell}(t)$ are $\Delta_{2}$-dual each other.

Since $c_{2}=c_{1}-c_{4}=0$ hold, the surface $\boldsymbol{X}_{\ell}^{h}$ is horo-flat in the sense of [22]. Moreover if we assume $c_{3}(t)=0$ for any $t$, then the singular value of $\boldsymbol{X}_{\ell}^{h}$ is $\boldsymbol{a}_{0}(t)$. We also consider the $\Delta_{1}$ and $\Delta_{2}$-dual surfaces of $\boldsymbol{a}_{0}$. By the same computations as those of the previous paragraph for obtaining the surface $\boldsymbol{X}_{\ell}^{h}$, we have the $\Delta_{1}$-dual surface $\boldsymbol{X}_{h}^{d}$ and $\Delta_{3}$-dual surface $\boldsymbol{X}_{h}^{\ell}$ of $\boldsymbol{a}_{2}$ as follows:

$$
\boldsymbol{X}_{h}^{d}(s, t):=\cos s \boldsymbol{a}_{2}(t)+\sin s \boldsymbol{a}_{3}(t) \quad \text { and } \quad \boldsymbol{X}_{h}^{\ell}(s, t):=\boldsymbol{a}_{0}(t)+\cos s \boldsymbol{a}_{2}(t)+\sin s \boldsymbol{a}_{3}(t) .
$$

In [22], we introduced these surfaces $\boldsymbol{X}_{\ell}^{h}$ and $\boldsymbol{X}_{h}^{\ell}$ by the same construction as the above and investigated the geomeotric properties and singularities of them. It has been shown in [22] that $\boldsymbol{X}_{\ell}^{h}$ is a linear Weingarten surface of non-Bryant type.

(2) $\Delta_{3}$-dual surface of $\boldsymbol{\ell}$ : Under the condition $c_{2}(t)=c_{3}(t)=0$ for any $t$, we consider a hight function $F: S_{1}^{3} \times I \longrightarrow \mathbb{R}$ defined by $F(X, t)=\langle X, \ell(t)\rangle-1$. By the same computations as those for detecting $\boldsymbol{X}_{\ell}^{h}(s, t)$, the descriminant set is given by

$$
X=\boldsymbol{a}_{2}(t)+s \boldsymbol{a}_{1}(t)-\frac{s^{2}}{2} \boldsymbol{\ell}(t)
$$

which we denote that $\boldsymbol{X}_{\ell}^{d}(s, t)$. Like as in the case for $\boldsymbol{X}_{\ell}^{h}$, we consider the dual surfaces of $\boldsymbol{a}_{2}$ here. By exactly the same calculations as those in the previous cases, the $\Delta_{1}$-dual surface $\boldsymbol{X}_{d}^{h}$ of $\boldsymbol{a}_{2}(t)$ and the $\Delta_{3}$-dual surface $\boldsymbol{X}_{d}^{\ell}$ of $\boldsymbol{a}_{2}(t)$ are parameterized by

$$
\boldsymbol{X}_{d}^{h}(s, t):=\cosh s \boldsymbol{a}_{0}(t)+\sinh s \boldsymbol{a}_{3}(t) \quad \text { and } \quad \boldsymbol{X}_{d}^{\ell}(s, t):=\boldsymbol{a}_{2}(t)+\cosh s \boldsymbol{a}_{0}(t)+\sinh s \boldsymbol{a}_{3}(t) .
$$

(3) $\Delta_{4}$-dual surface of $\boldsymbol{\ell}$ : Under the condition $c_{2}(t)=c_{6}(t)=0$ for any $t$, we consider a hight function $F: L C^{*} \times I \longrightarrow \mathbb{R}$ defined by $F(X, t)=\langle X, \ell(t)\rangle+2$. By exactly the same computations as those of the previous two cases. we have

$$
X=\boldsymbol{a}_{0}(t)-\boldsymbol{a}_{2}(t)+2 s \boldsymbol{a}_{1}(t)+s^{2} \boldsymbol{\ell}(t),
$$

which we denote $\boldsymbol{X}_{\ell}^{\ell}(s, t)$. We study geometric properties of $\boldsymbol{X}_{\ell}^{\ell}(s, t)$ in section 7 and investigate the singularities in section 8. Like as in the case of $\boldsymbol{X}_{\ell}^{h}$ and $\boldsymbol{X}_{\ell}^{d}$, we consider the dual surfaces of $\boldsymbol{\ell}_{-}:=\boldsymbol{a}_{0}-\boldsymbol{a}_{2}$. Under the condition $c_{2}(t)=c_{1}(t)+c_{4}(t)=0$ for any $t$, the $\Delta_{2}$-dual surface $\boldsymbol{X}_{\ell-}^{h}$ of $\boldsymbol{\ell}_{-}(t)$, the $\Delta_{3}$-dual surface $\boldsymbol{X}_{\ell_{-}}^{d}$ of $\boldsymbol{\ell}_{-}(t)$ and the $\Delta_{4}$-dual surface $\boldsymbol{X}_{\ell_{-}}^{\ell}$ of $\boldsymbol{\ell}_{-}(t)$ are parameterized by

$$
\begin{aligned}
\boldsymbol{X}_{\ell-}^{h}(s, t) & =\boldsymbol{a}_{0}(t)+s \boldsymbol{a}_{1}(t)+\frac{s^{2}}{2} \boldsymbol{\ell}_{-}(t), \\
\boldsymbol{X}_{\ell-}^{d}(s, t) & =-\boldsymbol{a}_{2}(t)+s \boldsymbol{a}_{1}(t)-\frac{s^{2}}{2} \boldsymbol{\ell}(t), \\
\boldsymbol{X}_{\ell-}^{\ell}(s, t) & =\boldsymbol{a}_{0}(t)+\boldsymbol{a}_{2}(t)+2 s \boldsymbol{a}_{1}(t)+s^{2} \boldsymbol{\ell}_{-}(t) .
\end{aligned}
$$

Since we can obtain these surfaces $\boldsymbol{X}_{\ell-}^{h}, \boldsymbol{X}_{\ell-}^{d}$ and $\boldsymbol{X}_{\ell-}^{\ell}$ by translating $\boldsymbol{a}_{2} \mapsto-\boldsymbol{a}_{2}$, geometric properties of these surfaces are completely the same as those of $\boldsymbol{X}_{\ell}^{h}, \boldsymbol{X}_{\ell}^{d}$ and $\boldsymbol{X}_{\ell}^{\ell}$. Here we remark the super and lower subscript. For example, $\boldsymbol{X}_{\ell}^{h}$ means this surface is the dual surface of a curve in the lightcone and lies in the hyperbolic 3 -space. Since surfaces $\boldsymbol{X}_{\ell}^{h}, \quad \boldsymbol{X}_{\ell}^{d}$ and $\quad \boldsymbol{X}_{\ell}^{\ell}$ are one-parameter families of parabolas, we call these surfaces parabollatic surfaces. If we adopt the 
word "parabolic" instead of the word "parabollatic", it might be confused with other notions. Now, we summerize the correspondences between these curves and surfaces:

$$
\begin{aligned}
& L C^{*} \supset \boldsymbol{\ell}(t) \longleftrightarrow \boldsymbol{X}_{\ell}^{h}(s, t)=\boldsymbol{a}_{0}(t)+s \boldsymbol{a}_{1}(t)+\frac{s^{2}}{2} \boldsymbol{\ell}(t) \quad \subset H_{+}^{3}(-1) \\
& L C^{*} \supset \boldsymbol{\ell}(t) \longleftrightarrow \boldsymbol{X}_{\ell}^{d}(s, t)=\boldsymbol{a}_{2}(t)+s \boldsymbol{a}_{1}(t)-\frac{s^{2}}{2} \boldsymbol{\ell}(t) \quad \subset S_{1}^{3} \\
& L C^{*} \supset \boldsymbol{\ell}(t) \longleftrightarrow \boldsymbol{X}_{\ell}^{l}(s, t)=\boldsymbol{a}_{0}(t)-\boldsymbol{a}_{2}(t)+2 s \boldsymbol{a}_{1}(t)+s^{2} \boldsymbol{\ell}(t) \quad \subset L C^{*} \\
& H_{+}^{3}(-1) \supset \boldsymbol{a}_{0}(t) \longleftrightarrow \boldsymbol{X}_{h}^{d}(s, t)=\cos s \boldsymbol{a}_{2}(t)+\sin s \boldsymbol{a}_{3}(t) \quad \subset S_{1}^{3} \\
& H_{+}^{3}(-1) \supset \boldsymbol{a}_{0}(t) \longleftrightarrow \boldsymbol{X}_{h}^{\ell}(s, t)=\boldsymbol{a}_{0}(t)+\cos s \boldsymbol{a}_{2}(t)+\sin s \boldsymbol{a}_{3}(t) \quad \subset L C^{*} \\
& S_{1}^{3} \supset \boldsymbol{a}_{2}(t) \longleftrightarrow \boldsymbol{X}_{d}^{h}(s, t)=\cosh s \boldsymbol{a}_{0}(t)+\sinh s \boldsymbol{a}_{3}(t) \quad \subset H_{+}^{3}(-1) \\
& S_{1}^{3} \supset \boldsymbol{a}_{2}(t) \longleftrightarrow \boldsymbol{X}_{d}^{\ell}(s, t)=\boldsymbol{a}_{2}(t)+\cosh s \boldsymbol{a}_{0}(t)+\sinh s \boldsymbol{a}_{3}(t) \subset L C^{*} .
\end{aligned}
$$

\subsection{Dualities of "flat"surfaces}

By using the equations for the pseudo-orthonormal frame, we have

$$
\begin{aligned}
& \left(\boldsymbol{X}_{\ell}^{h}\right)^{\prime}(s, t)=s c_{1} \boldsymbol{a}_{0}+c_{1} \boldsymbol{a}_{1}+s c_{4} \boldsymbol{a}_{2}+\left(c_{3}+s c_{5}+\frac{s^{2}}{2} c_{36}\right) \boldsymbol{a}_{3} \\
& \left(\boldsymbol{X}_{\ell}^{h}\right)_{s}(s, t)=s \boldsymbol{a}_{0}+\boldsymbol{a}_{1}+s \boldsymbol{a}_{2},
\end{aligned}
$$

where ()$^{\prime}$ means $\partial / \partial t$ and ()$_{s}$ means $\partial / \partial s$. It follows that we have

$$
\begin{aligned}
& \left\langle\boldsymbol{X}_{\ell}^{h}( \pm s, t), \boldsymbol{X}_{\ell}^{d}(\mp s, t)\right\rangle \equiv 0, \\
& \quad\left\langle\left(\boldsymbol{X}_{\ell}^{h}\right)^{\prime}( \pm s, t), \boldsymbol{X}_{\ell}^{d}(\mp s, t)\right\rangle \equiv 0 \text { and }\left\langle\left(\boldsymbol{X}_{\ell}^{h}\right)_{s}( \pm s, t), \boldsymbol{X}_{\ell}^{d}(\mp s, t)\right\rangle \equiv 0,
\end{aligned}
$$

where $\equiv 0$ means that it holds identically. This implies that $\left(\boldsymbol{X}_{\ell}^{h}, \boldsymbol{X}_{\ell}^{d}\right): \mathbb{R}^{2} \rightarrow \Delta_{1}$ is an isotropic map with respect to $K_{1}$. Therefore $\boldsymbol{X}_{\ell}^{h}$ and $\boldsymbol{X}_{\ell}^{d}$ are $\Delta_{1}$-dual each other. Since $\boldsymbol{X}_{\ell}^{h}(s, t)$ is a linear Weingarten surface of non-Bryant type, $\boldsymbol{X}_{\ell}^{d}(s, t)$ is a linear Weingarten surface of non-Bianchi type by Theorem 5.3. By the same calculation, we can show that the $\Delta_{2}$-duality between $\boldsymbol{X}_{\ell}^{h}( \pm s, t)$ and $\boldsymbol{X}_{\ell}^{\ell}( \pm s, t)$, and the $\Delta_{3}$-duality between $\boldsymbol{X}_{\ell}^{d}( \pm s, t)$ and $-\boldsymbol{X}_{\ell}^{\ell}(\mp s, t)$ under the assumptions $c_{2}(t) \equiv 0, c_{1}(t)-c_{4}(t) \equiv 0$. These assumptions mean that a kind of flatness of $\boldsymbol{X}_{\ell}^{h}(s, t), \boldsymbol{X}_{\ell}^{d}(s, t)$ and $\boldsymbol{X}_{\ell}^{\ell}(s, t)$. For $\boldsymbol{X}_{\ell}^{h}(s, t)$, such a flatness is called horo-flat in [22].

Furthermore, under the conditions $c_{2}(t)=c_{3}(t)=0$ for any $t\left(\right.$ resp. $\left.c_{2}(t)=c_{6}(t)=0\right)$, we have $\left\langle\boldsymbol{X}_{h}^{d}, \boldsymbol{X}_{h}^{\ell}\right\rangle=1$ (resp. $\left.\left\langle\boldsymbol{X}_{d}^{h}, \boldsymbol{X}_{d}^{\ell}\right\rangle=-1\right)$ and $\left\langle\boldsymbol{X}_{h}^{d}, d \boldsymbol{X}_{h}^{\ell}\right\rangle=0$ (resp. $\left\langle\boldsymbol{X}_{d}^{h}, d \boldsymbol{X}_{d}^{\ell}\right\rangle=0$ ). Hence $\boldsymbol{X}_{h}^{d}$ and $\boldsymbol{X}_{h}^{\ell}$ are $\Delta_{3}$-dual (resp. $\boldsymbol{X}_{d}^{h}$ and $\boldsymbol{X}_{d}^{\ell}$ are $\Delta_{2}$-dual) each other. By Theorem 5.3 and the mandala of Legendrian dualities, the surface $\boldsymbol{X}_{\ell}^{\ell}(s, t)$ corresponds to the linear Weingarten surfaces of non-Bryant type in $H_{+}^{3}(-1)$ and of non-Bianchi type in $S_{1}^{3}$.

Thus we have the following diagram which expresses the duality for flat surfaces in pseudospheres: 


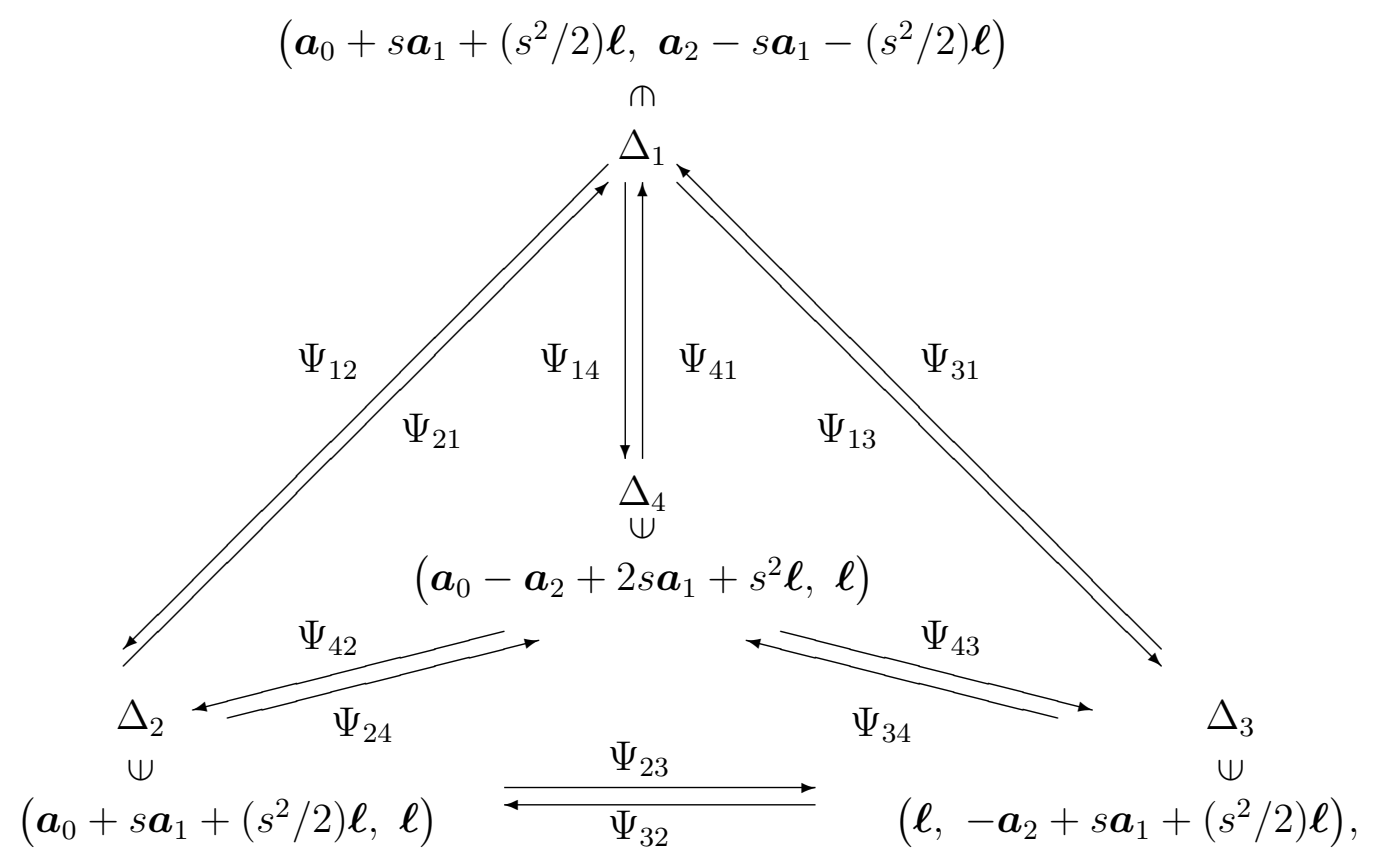

If we start from a curve $\boldsymbol{\ell}$ in the lightcone, we have the following diagram of dualities:

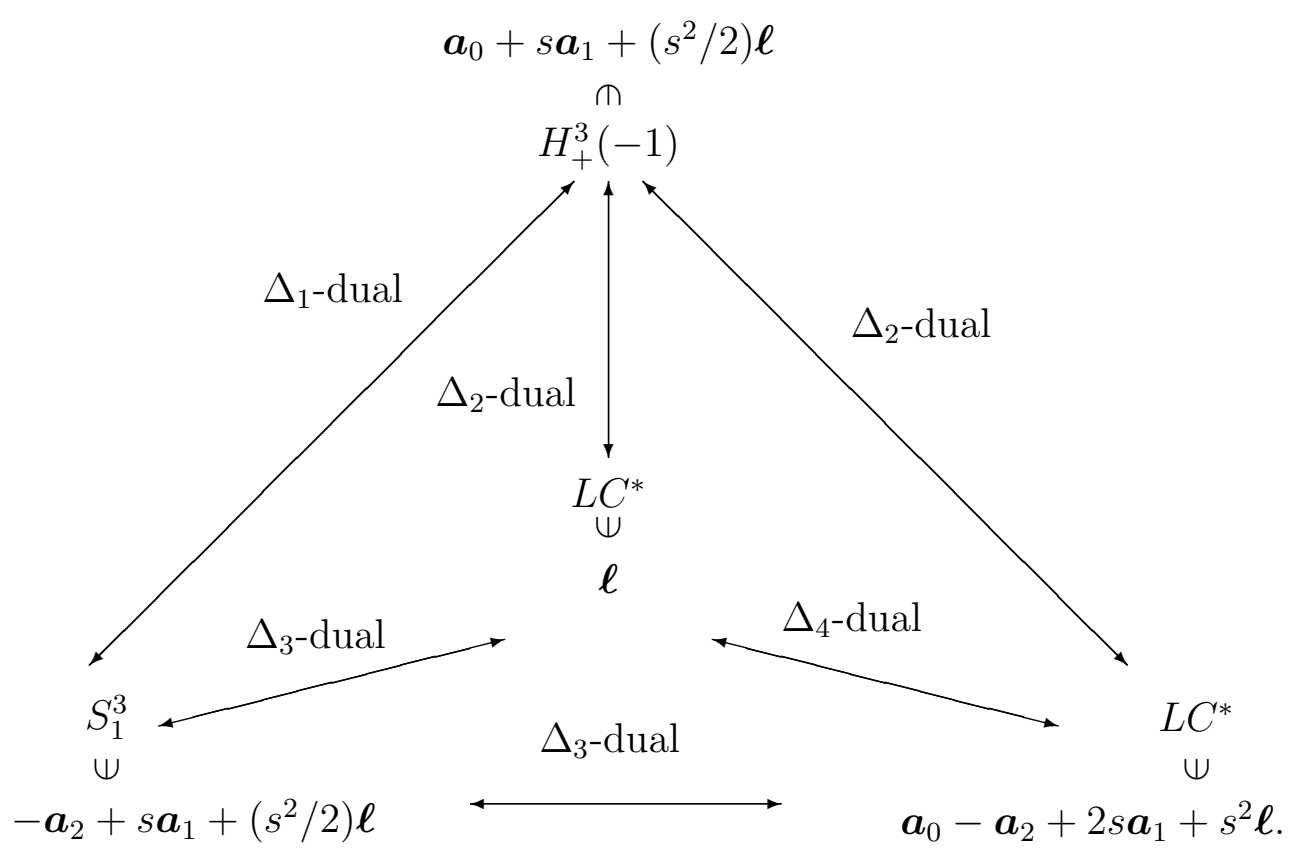

Also we can have the diagram on dualities starting from $\boldsymbol{a}_{0}$ and $\boldsymbol{a}_{2}$ :

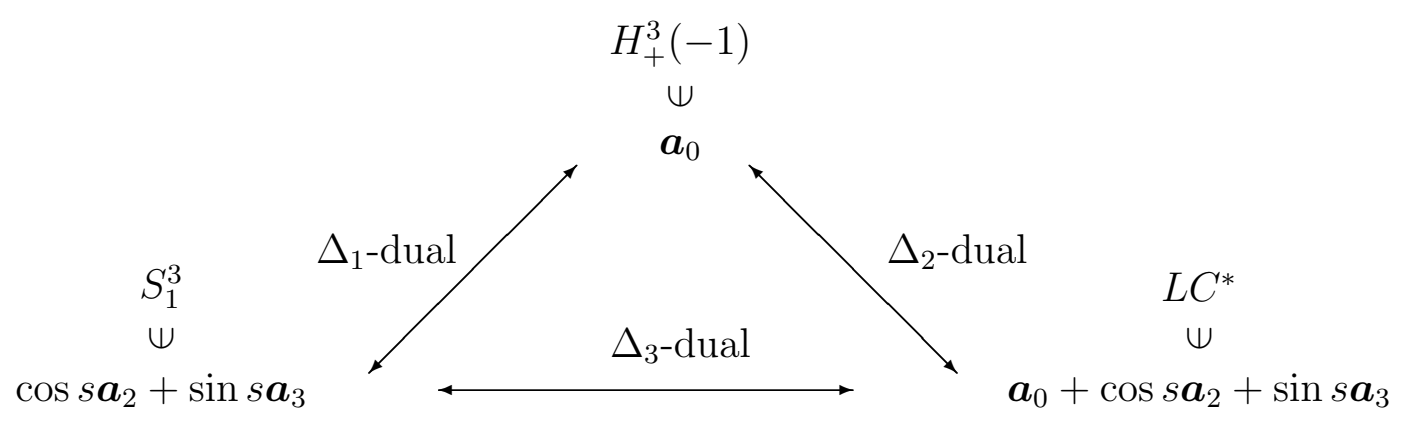

and 


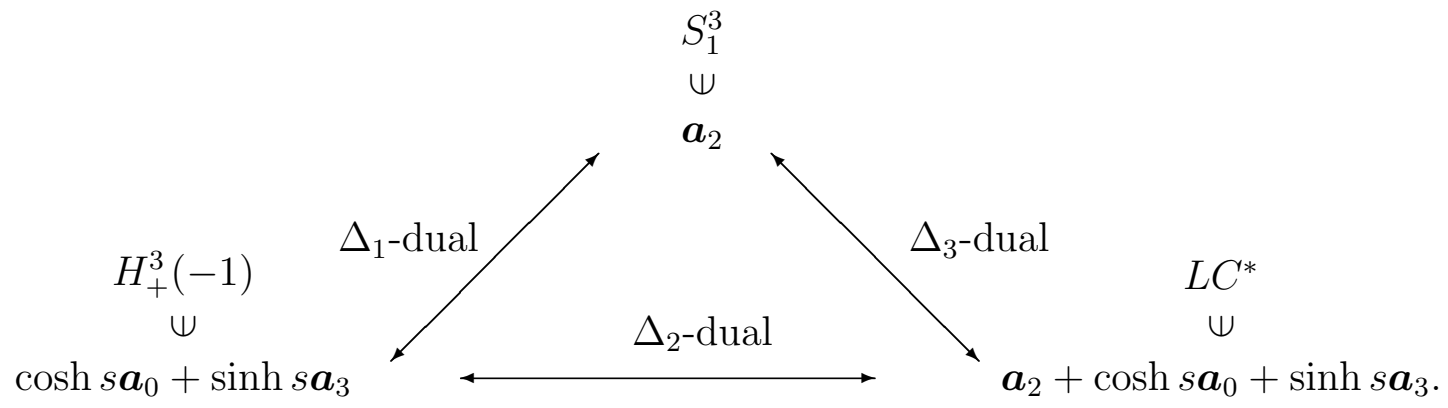

We can also have a diagram starting from the curve $\boldsymbol{\ell}_{-}=\boldsymbol{a}_{0}-\boldsymbol{a}_{2}$. However, the situation is the same as the case for $\ell$, so that we omit it.

\section{$7 \quad$ Fundamental properties of parabollatic surfaces}

In section 6 , we construct the dual surfaces of $\boldsymbol{\ell}$ which are called parabollatic surfaces. The analogous notion in Euclidean space is ruled surfaces given by one-parameter families of lines in $\mathbb{R}^{3}$. For the study of singularities and geometric properties of ruled surfaces, the striction curve plays a crucial role ([16]). The striction curve is a curve on the ruled surface which contains the singularities of the surface. Similarly, an analogous notion of the striction curve also plays a crucial role for one-parameter families of circles ([23]). Since surfaces $\boldsymbol{X}_{\ell}^{h}, \boldsymbol{X}_{\ell}^{d}$ and $\boldsymbol{X}_{\ell}^{\ell}$ are one-parameter families of parabolas, we try to find the analogous notion of striction curves of ruled surfaces. Here, we only consider the surfaces $\boldsymbol{X}_{\ell}^{h}, \boldsymbol{X}_{\ell}^{d}$ and $\boldsymbol{X}_{\ell}^{\ell}$. We remark that surfaces $\boldsymbol{X}_{h}^{d}, \boldsymbol{X}_{h}^{\ell}, \boldsymbol{X}_{d}^{h}$ and $\boldsymbol{X}_{d}^{\ell}$ have similar properties as the circular surfaces [23]. We shall investigate these surfaces in the forthcoming paper.

\subsection{The striction curve of $X_{\ell}^{d}$}

Let $A=\left(\boldsymbol{a}_{0}, \boldsymbol{a}_{1}, \boldsymbol{a}_{2}, \boldsymbol{a}_{3}\right): I \rightarrow \mathfrak{s o}(3,1)$ be a pseudo-orthonormal frame defined in Section 6 . The $\Delta_{3}$-dual surface $\boldsymbol{X}_{\ell}^{d}$ of $\boldsymbol{\ell}$ is defined by

$$
\boldsymbol{X}_{\ell, A}^{d}(s, t):=\boldsymbol{a}_{2}(t)+s \boldsymbol{a}_{1}(t)-\frac{s^{2}}{2} \boldsymbol{\ell}(t) .
$$

For any $t$, the curve $s \mapsto \boldsymbol{X}_{\ell, A}^{d}(s, t)$ is a parabola. The each parabola called the generating parabola.

On the other hand, for any curve

$$
\overline{\boldsymbol{a}_{2}}(t)=\boldsymbol{a}_{2}(t)+s(t) \boldsymbol{a}_{1}(t)-\frac{s(t)^{2}}{2} \boldsymbol{\ell}(t)
$$

on the $\boldsymbol{X}_{\ell}^{d}$, we define

$$
\begin{aligned}
\overline{\boldsymbol{a}_{0}}(t)=\left(1+\frac{s(t)^{2}}{2}\right) \boldsymbol{a}_{0}(t)-s(t) \boldsymbol{a}_{1}(t)+\frac{s(t)^{2}}{2} \boldsymbol{a}_{2}(t), \\
\overline{\boldsymbol{a}_{1}}(t)=-s(t) \boldsymbol{a}_{0}(t)+\boldsymbol{a}_{1}(t)-s \boldsymbol{a}_{2}(t) \text { and } \overline{\boldsymbol{a}_{3}}(t)=\boldsymbol{a}_{3}(t)
\end{aligned}
$$

then $\boldsymbol{X}_{\ell, \bar{A}}^{d}(s-s(t), t)=\boldsymbol{X}_{\ell, A}^{d}(s, t)$ holds. Moreover, we define invariants $\bar{C}(t)$ by the formula 
$\bar{A}^{\prime}(t)=\bar{C}(t) \bar{A}(t)$, then we have

$$
\left\{\begin{array}{l}
\overline{c_{1}}=\left(\frac{1-s(t)^{2}}{2}\right) c_{1}-s^{\prime}(t)+\frac{s(t)^{2}}{2} c_{4}-s(t) c_{2} \\
\overline{c_{2}}=s(t) c_{1}+c_{2}-s(t) c_{4} \\
\overline{c_{3}}=c_{3}-s(t) c_{5}+\frac{s(t)^{2}}{2} c_{36} \\
\overline{c_{4}}=\frac{-s(t)^{2}}{2} c_{1}-s(t) c_{2}+\left(1+\frac{s(t)^{2}}{2}\right) c_{4}-s^{\prime}(t) \\
\overline{c_{5}}=c_{5}-s(t) c_{36} \\
\overline{c_{6}}=c_{6}+s(t) c_{5}-\frac{s(t)^{2}}{2} c_{36} .
\end{array}\right.
$$

It follows that

$$
\overline{c_{1}}-\overline{c_{4}}=c_{1}-c_{4}
$$

and

$$
\overline{c_{1}}-\overline{c_{4}}=\overline{c_{2}}=0 \text { if and only if } c_{1}-c_{4}=c_{2}=0 .
$$

This means that the condition $c_{1}-c_{4}=c_{2}=0$ is invariant under the adopted coordinate changes. Here, a reparameterization $(s, t) \mapsto(S, T)$ of $\boldsymbol{X}_{\ell, A}^{d}$ is said to be adopted if $S=s-s(t)$ and $T=t$. We have the following proposition.

Proposition 7.1. Let $\boldsymbol{X}_{\ell, A}^{d}$ be a parameterization of a parabollatic surfaces of the form

$$
\boldsymbol{X}_{\ell, A}^{d}(s, t)=\boldsymbol{a}_{2}+s \boldsymbol{a}_{1}-\frac{s^{2}}{2} \boldsymbol{\ell}
$$

such that $c_{1}-c_{4}$ never vanish. Then Image $\boldsymbol{X}_{\ell, A}^{d}$ has an adopted reparameterization of the form

$$
\boldsymbol{X}_{\ell, \bar{A}}^{d}(s, t)=\overline{\boldsymbol{a}}_{2}+s \overline{\boldsymbol{a}}_{1}-\frac{s^{2}}{2} \overline{\boldsymbol{\ell}}
$$

satisfying $\left\langle{\overline{\boldsymbol{a}_{0}}}^{\prime}, \overline{\boldsymbol{a}_{2}}\right\rangle=0$ for any $t$.

Proof. Let us define

$$
s(t)=\frac{-c_{2}(t)}{c_{1}(t)-c_{4}(t)}
$$

and define curves $\overline{\boldsymbol{a}}_{0}, \overline{\boldsymbol{a}}_{1}, \overline{\boldsymbol{a}}_{2}$ by (7.1) and (7.2). Then $\overline{c_{2}}=0$ holds. We do not need to say that $\boldsymbol{X}_{\ell, \bar{A}}^{d}$ and $\boldsymbol{X}_{\ell, A}^{d}$ have the same image. Thus the condition of the proposition holds.

A curve $\boldsymbol{X}_{\ell, A}^{d}(s(t), t)$ on the surface is called striction curve if $\left\langle\boldsymbol{a}_{0}^{\prime}(t), \boldsymbol{a}_{2}(t)\right\rangle=0$ for any $t$ holds. Proposition 7.1 implies that we can take $\boldsymbol{a}_{2}$ as the striction curve. Singularities of parabollatic surfaces are located on the striction curve. For any parabollatic surfaces satisfying $c_{1}-c_{4}=0$, there exists a unique striction curve.

Proposition 7.2. Let $\boldsymbol{X}_{\ell, A}^{d}$ be a parabollatic surface with the striction curve $\boldsymbol{a}_{2}$ and $c_{1}-c_{4} \neq 0$. If $\left(s_{0}, t_{0}\right)$ is a singular point, then $s_{0}=0$ namely, $x_{0}$ is located on the striction curve. Moreover, if $\left(0, t_{0}\right)$ is a singular point, them the generating parabola at $t_{0}$ is tangent to the striction curve.

Proof. Direct calculation and $\boldsymbol{a}_{2}^{\prime}=-c_{4} \boldsymbol{a}_{1}$ yield the conclusion. 


\subsection{The striction curve of $X_{\ell}^{\ell}$}

In this section, we study general properties of dual surfaces of $\boldsymbol{\ell}$. Let $A=\left(\boldsymbol{a}_{0}, \boldsymbol{a}_{1}, \boldsymbol{a}_{2}, \boldsymbol{a}_{3}\right)$ : $I \rightarrow \mathfrak{s o}(3,1)$ be a pseudo-orthonormal frame defined in Section 6 . The dual surface $\boldsymbol{X}_{\ell}^{\ell}$ of $\boldsymbol{\ell}$ is defined by

$$
\boldsymbol{X}_{\ell, A}^{\ell}(s, t):=\boldsymbol{a}_{0}(t)-\boldsymbol{a}_{2}(t)+2 s \boldsymbol{a}_{1}(t)+s^{2} \boldsymbol{\ell}(t) .
$$

For any curve, $\overline{\boldsymbol{a}_{0}}-\overline{\boldsymbol{a}_{2}}(t)=\boldsymbol{a}_{0}-\boldsymbol{a}_{2}(t)+2 s(t) \boldsymbol{a}_{1}(t)+s^{2} \boldsymbol{\ell}(t)$ on $\boldsymbol{X}_{\ell}^{\ell}, \boldsymbol{a}$, we define

$$
\begin{gathered}
\overline{\boldsymbol{a}_{0}}(t)=\boldsymbol{a}_{0}+s(t) \boldsymbol{a}_{1}+\frac{s(t)^{2}}{2} \boldsymbol{\ell}, \quad \overline{\boldsymbol{a}_{1}}(t)=\boldsymbol{a}_{1}+s(t) \boldsymbol{\ell}(t) \\
\overline{\boldsymbol{a}_{2}}(t)=\boldsymbol{a}_{2}-s(t) \boldsymbol{a}_{1}-\frac{s(t)^{2}}{2} \boldsymbol{\ell} \text { and } \overline{\boldsymbol{a}_{3}}(t)=\boldsymbol{a}_{3}(t)
\end{gathered}
$$

then $\boldsymbol{X}_{\ell, \bar{A}}^{\ell}(s-s(t), t)=\boldsymbol{X}_{\ell, A}^{\ell}(s, t)$ holds. Moreover, we define invariants $\bar{C}(t)$ by the formula $\bar{A}^{\prime}(t)=\bar{C}(t) \bar{A}(t)$, then we have

$$
\left\{\begin{array}{l}
\overline{c_{1}}=-\frac{s(t)^{2}}{2}\left(c_{1}-c_{4}\right)+c_{1}+s^{\prime}(t)+s c_{2} \\
\overline{c_{2}}=-s(t) c_{1}+c_{2}+s(t) c_{4} \\
\overline{c_{3}}=c_{3}+s(t) c_{5}+\frac{s(t)^{2}}{2} c_{36} \\
\overline{c_{4}}=-\frac{s(t)^{2}}{2} c_{1}+s(t) c_{2}+\frac{s(t)^{2}}{2} c_{4}+s^{\prime}(t) \\
\overline{c_{5}}=c_{5}-s(t) c_{36} \\
\overline{c_{6}}=c_{6}-s(t) c_{5}-\frac{s(t)^{2}}{2} c_{36}
\end{array}\right.
$$

Thus it follows that

$$
\overline{c_{1}}-\overline{c_{4}}=c_{1}-c_{4}
$$

and

$$
\overline{c_{1}}-\overline{c_{4}}=\overline{c_{2}}=0 \text { if and only if } c_{1}-c_{4}=c_{2}=0 .
$$

A reparameterization $(s, t) \mapsto(S, T)$ of $\boldsymbol{X}_{\ell, A}^{\ell}$ is said to be adopted if $S=s-s(t)$ and $T=t$. We have the following proposition.

Proposition 7.3. Let $\boldsymbol{X}_{\ell, A}^{\ell}$ be a parameterization of a parabollatic surfaces of the form

$$
\boldsymbol{X}_{\ell, A}^{\ell}(s, t)=\boldsymbol{a}_{0}-\boldsymbol{a}_{2}+2 s \boldsymbol{a}_{1}+s^{2} \boldsymbol{\ell}
$$

such that $c_{1}-c_{4}$ never vanish. Then Image $\boldsymbol{X}_{\ell, A}^{\ell}$ has an adopted reparameterization of the form

$$
\boldsymbol{X}_{\ell, \bar{A}}^{\ell}(s, t)=\overline{\boldsymbol{a}_{0}}-\overline{\boldsymbol{a}}_{2}+2 s \overline{\boldsymbol{a}}_{1}+s^{2} \overline{\boldsymbol{\ell}}
$$

satisfying $\left\langle\overline{\boldsymbol{a}_{0}}, \overline{\boldsymbol{a}_{2}}\right\rangle=0$ for any $t$.

Proof. Let us define

$$
s(t)=\frac{c_{2}(t)}{c_{1}(t)-c_{4}(t)}
$$


and define curves $\bar{A}$ as (7.1) and (7.2). Then $\overline{c_{2}}=0$ holds. We do not need to say that $\boldsymbol{X}_{\ell, \bar{A}}^{\ell}$ and $\boldsymbol{X}_{\ell, A}^{\ell}$ have the same image. Thus the condition of the proposition holds.

A curve on the surface $\boldsymbol{X}_{\ell, A}^{\ell}(s(t), t)$ is called striction curve if $\left\langle\boldsymbol{a}_{0}^{\prime}(t), \boldsymbol{a}_{2}(t)\right\rangle=0$ for any $t$ holds. Proposition 7.3 implies that one can take $\boldsymbol{a}_{2}$ as the striction curve. Singularities of parabollatic surfaces are located on the striction curve.

Proposition 7.4. Let $\boldsymbol{X}_{\ell, A}^{\ell}$ be a parabollatic surface with the striction curve $\boldsymbol{a}_{2}$ and $c_{1}-c_{4} \neq 0$. If $\left(s_{0}, t_{0}\right)$ is a singular point then $s_{0}=0$ namely, $x_{0}$ is located on the striction curve. Moreover, if $\left(0, t_{0}\right)$ is a singular point then the generating parabola at $t_{0}$ which is tangent to the striction curve.

Proof. For a parabollatic surface $\boldsymbol{X}_{\ell, A}^{\ell}$, point $\left(s_{0}, t_{0}\right)$ is a singular point if and only if

$$
c_{2}\left(t_{0}\right)-s_{0}\left(c_{1}\left(t_{0}\right)-c_{4}\left(t_{0}\right)\right)=0 \text { and } c_{3}\left(t_{0}\right)-c_{6}\left(t_{0}\right)+2 s_{0} c_{5}\left(t_{0}\right)+s_{0}^{2} c_{36}\left(t_{0}\right)=0 .
$$

Thus if $\boldsymbol{a}_{0}-\boldsymbol{a}_{2}$ is the striction curve, them $s_{0}=0$ and $c_{3}\left(t_{0}\right)-c_{6}\left(t_{0}\right)=0$ holds. Moreover, if $c_{3}\left(t_{0}\right)-c_{6}\left(t_{0}\right)=0$, then the parabola tangents to the striction curve at $\left(0, t_{0}\right)$. Because of $\boldsymbol{a}_{0}^{\prime}-\boldsymbol{a}_{2}^{\prime}=-c_{2}\left(\boldsymbol{a}_{0}-\boldsymbol{a}_{2}\right)+\left(c_{1}+c_{4}\right) \boldsymbol{a}_{1}$.

Singularities of these surface are studied in Section 8. Although we can costruct dual surfaces from $\boldsymbol{\ell}_{-}$, their geometric properties are the same as those of dual surfaces constructed from $\boldsymbol{\ell}$, so that we omit the study of their striction curves.

\section{Singularities of flat parabollatic surfaces}

\subsection{Criteria for singularities of frontals}

All surfaces investigating here have an isotropic lift to some contact manifold. They are called frontals which are originally investigated by Zakalyukin $[34,35]$. In order to investigate singularities of concretely parameterized surfaces, the identification problems for singularities are important. Let $f_{0}$ be a given map germs. The identification problem for $f_{0}$ means that finding a necessary and sufficient condition that if a map germ $f$ satisfies the condition, then $f$ to be $\mathcal{A}$-equivalent to $f_{0}$. We call the condition criterion of $f_{0}$. Such criteria are given by many people now. Simple criteria for cuspidal edge and swallowtail were given by Kokubu, Rossman, Saji, Umehara and Yamada [28]. Other criteria for singularities of frontals are investigated in $[13,32,22]$. Here, we briefly review the criteria of frontals. Let $\pi: E \rightarrow M$ be a Legendrian fibration from a five-dimensional contact manifold $E$ to a three-dimensional manifold $M$. A $C^{\infty}$-map $f: U \rightarrow M$ is called a frontal (resp. front) if there exists an isotropic lift (resp. Legendrian immersion) $L_{f}: U \rightarrow E$, where $U \subset \mathbb{R}^{2}$ be an open set. Recall that the image of the Legendrian sumbanifold is called the wavefront set (see Section 3). By the generalized Darboux theorem (cf., [2], 20.3), any Legendrian fibration $E \rightarrow M$ is locally equivalent to the standard fibration $P T \mathbb{R}^{3} \rightarrow \mathbb{R}^{3}$. Therefore, we assume that $E \rightarrow M$ is $P T \mathbb{R}^{3} \rightarrow \mathbb{R}^{3}$ and that $f$ is a $C^{\infty}$ map germ $(U, p) \rightarrow\left(\mathbb{R}^{3}, f(p)\right)$. Taking the fiber component, let us denote $L_{f}=(f,[\nu])$. The discriminant function of a frontal $f$ is defined by $\lambda(u, v)=\operatorname{det}\left(f_{u}, f_{v}, \nu\right)(u, v)$ using the coordinate system $(u, v)$ on $U$, where $f_{u}=\partial f / \partial u$, for example. A singular point $p$ of $f$ is non-degenerate if $d \lambda(p) \neq \mathbf{0}$ holds. Let $p$ be a non-degenerate singular point of a frontal $f$. In this case, there exists a smooth parameterization $\gamma(t):(-\varepsilon, \varepsilon) \rightarrow U, \gamma(0)=p$ of $S(f)$ near 
$p$. Moreover, there exists a smooth vector field $\eta(t)$ along $\gamma$ satisfying that $\eta(t)$ generates the kernel of $d f_{\gamma(t)}$. We call this vector field the null vector field. Now we define a function $\phi_{f}(t)$ on $\gamma$ by

$$
\phi_{f}(t)=\operatorname{det}\left((f \circ \gamma)^{\prime}, \nu \circ \gamma, d \nu(\eta)\right)(t)
$$

Using these notations, the following criteria have been obtained.

Theorem 8.1. [28, 13] Let $f: U \rightarrow \mathbb{R}^{3}$ be a front and $p$ a non-degenerate singular point of $f$ and $\gamma:(\varepsilon, \varepsilon) \rightarrow U, \gamma(0)=p$ be a smooth parameterization of $S(f)$ near $p$. Then the following assertions hold.

- If $\eta \lambda(p) \neq 0$ then $f$ to be a front near $p$ if and only if $\phi_{f}(0) \neq 0$ holds.

- $\quad$ The map germ $f$ at $p$ is $\mathcal{A}$-equivalent to the cuspidal edge if and only if $f$ to be a front near $p$ and $\eta \lambda(p) \neq 0$ hold.

- $\quad$ The map germ $f$ at $p$ is $\mathcal{A}$-equivalent to the swallowtail if and only if $f$ to be a front near $p, \eta \lambda(p)=0$ and $\eta \eta \lambda(p) \neq 0$.

- $\quad$ The map germ $f$ at $p$ is $\mathcal{A}$-equivalent to the cuspidal cross cap if and only if $\eta \lambda(p) \neq 0$, $\phi_{f}(0)=0$ and $\phi_{f}^{\prime}(0) \neq 0$.

Here, $\eta \lambda: U \rightarrow \mathbb{R}$ means the directional derivative of $\lambda$ by the vector field $\tilde{\eta}$, where $\tilde{\eta} \in \mathfrak{X}(U)$ is an extended vector field of $\eta$ to $U$. Moreover, we have the following criterion for the cuspidal butterfly.

Theorem 8.2. Let $f: U \rightarrow \mathbb{R}^{3}$ be a front and $p$ a non-degenerate singular point of $f$ and $\gamma:(\varepsilon, \varepsilon) \rightarrow U, \gamma(0)=p$ be a smooth parameterization of $S(f)$ near $p$. Then the map germ $f$ at $p$ is $\mathcal{A}$-equivalent to the cuspidal butterfly if and only if $f$ to be a front near $p$ and $\eta \lambda(p)=$ $\eta \eta \lambda(p)=0$ and $\eta \eta \eta \lambda(p) \neq 0$.

A proof of this theorem is given in the appendix. Next we consider a degenerate singularity. Let $p$ be a degenerate singularity of a front $f$. If $\operatorname{rank}(d f)_{p}=1$, then there exists a non-zero vector field $\eta$ near $p$ such that if $q \in S(f)$ then $\eta(q)$ generates the kernel of $d f(q)$. A criterion for the degenerate singularity is given as follows.

Theorem 8.3. [22] Let $f$ be a frontal and $p$ a degenerate singular point of $f$ Then the following assertions hold.

- $\quad$ The map germ $f$ at $p$ is $\mathcal{A}$-equivalent to the cuspidal lips if and only if $\operatorname{rank}(d f)_{p}=1$ and det $\operatorname{Hess} \lambda(p)>0$.

- $\quad$ The map germ $f$ at $p$ is $\mathcal{A}$-equivalent to the cuspidal beaks if and only if $\operatorname{rank}(d f)_{p}=1$, $\operatorname{det} \operatorname{Hess} \lambda(p)<0$ and $\eta \eta \lambda(p) \neq 0$.

In order to study singularities of a front in pseudo-Riemannian space, we introduce the following notion.

Definition 8.4. ([13]) A lift $L_{g}: U \rightarrow T^{*} N$ of a $C^{\infty}$-map $g: U \rightarrow N$ to be admissible if $g$ never intersect to the zero-section and $g_{*}\left(T_{p} U\right) \subset \operatorname{ker}\left(L_{g}(p)\right)$, where $\operatorname{ker}\left(L_{g}(p)\right) \subset T_{g(p)} N$ is the kernel of a linear map $L_{g}(p)$.

Using this notion, a criterion for cuspidal cross cap is stated as follows.

Theorem 8.5. ([13, Theorem 1.4]) Let $g: U \rightarrow N$ be a frontal and $L_{g}: U \rightarrow T^{*} N$ be an admissible lift of $g$. Let $D$ be an arbitrary linear connection on $N$. Suppose that $\gamma(t)$ 
$(|t|<\varepsilon)$ is a singular curve on $U$ passing through a non-degenerate singular point $p=\gamma(0)$, and $\xi_{g}:(-\varepsilon, \varepsilon) \rightarrow T N$ is an arbitrarily fixed vector field along $\gamma$ such that

(1) $L\left(\xi_{g}\right)$ vanishes on $U$ and

(2) $\xi_{g}$ is transversal to $g_{*}\left(T_{p} U\right)$ at $p$.

We define a function $\psi_{g}(t)$ by

$$
\psi_{g}(t)=L\left(D_{\eta(t)}^{g} \xi_{g}(\gamma(t))\right)
$$

where $\eta(t)$ is a null vector field on the singular curve parameterized by $t$. Then the germ $g$ at $p$ is $\mathcal{A}$-equivalent to the cuspidal cross cap if and only if $\psi_{g}(0)=0$ and $\psi_{g}^{\prime}(0) \neq 0$ hold, and $\eta(0)$ is transversal to $\gamma^{\prime}(0)$.

\subsection{Singularities of dual surfaces of $\ell$}

In this section, we apply the criteria, and describe the conditions of singularities of dual surfaces of $\boldsymbol{\ell}$. We assume that $c_{2} \equiv c_{1}-c_{4} \equiv 0$ in this section.

Theorem 8.6. The singular set of $\boldsymbol{X}_{\ell}^{h}$ is $S\left(\boldsymbol{X}_{\ell}^{h}\right)=\left\{(s, t) \mid 2 c_{3}(t)+2 s c_{5}(t)+s^{2} c_{36}(t)=0\right\}$ and $\boldsymbol{X}_{\ell}^{h}$ is a frontal for any $p_{0}=\left(s_{0}, t_{0}\right) \in S\left(\boldsymbol{X}_{\ell}^{h}\right)$. Then we have the following assertions:

- If $c_{36}\left(t_{0}\right) \neq 0$ holds, then $\boldsymbol{X}_{\ell}^{h}$ to be a front near $p_{0}$.

- $\quad \boldsymbol{X}_{\ell}^{h}$ at $p_{0}$ is $\mathcal{A}$-equivalent to the cuspidal edge if and only if $c_{36} \neq 0$ and $\alpha_{l}^{h}:=-2 c_{1}\left(c_{5}+\right.$ $\left.s c_{36}\right)+2 c_{3}^{\prime}+2 s c_{5}^{\prime}+s^{2} c_{36}^{\prime} \neq 0$ hold at $p_{0}$.

- $\quad \boldsymbol{X}_{\ell}^{h}$ at $p_{0}$ is $\mathcal{A}$-equivalent to the swallowtail if and only if $c_{36} \neq 0, c_{5}+s c_{36} \neq 0, \alpha_{l}^{h}=0$ and $c_{1}\left(\alpha_{l}^{h}\right)_{s}+\left(\alpha_{l}^{h}\right)^{\prime} \neq 0$ hold at $p_{0}$.

- $\quad \boldsymbol{X}_{\ell}^{h}$ at $p_{0}$ is $\mathcal{A}$-equivalent to the cuspidal butterfly if and only if $c_{36} \neq 0, c_{5}+s c_{36} \neq 0$, $\alpha_{l}^{h}=0, c_{1}\left(\alpha_{l}^{h}\right)_{s}+\left(\alpha_{l}^{h}\right)^{\prime} \neq 0$ and $c_{1}^{2}\left(\alpha_{l}^{h}\right)_{s s}+2 c_{1}\left(\alpha_{l}^{h}\right)_{s}^{\prime}+c_{1}^{\prime}\left(\alpha_{l}^{h}\right)_{s}+\left(\alpha_{l}^{h}\right)^{\prime \prime}=0$ hold at $p_{0}$.

- $\quad \boldsymbol{X}_{\ell}^{h}$ at $p_{0}$ is $\mathcal{A}$-equivalent to the cuspidal lips (resp. cuspidal beaks) if and only if $c_{36} \neq$ $0, c_{5}+s c_{36}=0,2 c_{3}^{\prime}+2 s c_{5}^{\prime}+s^{2} c_{36}^{\prime}=0$ and $\operatorname{det} H_{\ell}^{h}>0$ (resp. $\operatorname{det} H_{\ell}^{h}<0$ and $\left.-2 c_{1}\left(c_{36}+c_{5}^{\prime}+s c_{36}^{\prime}\right)+\left(-2 c_{1}\left(c_{5}+s c_{36}\right)+c_{3}^{\prime}+s c_{5}^{\prime}+s^{2} c_{36}^{\prime}\right)^{\prime} \neq 0\right)$ hold at $p_{0}$, where

$$
H_{\ell}^{h}=\left(\begin{array}{cc}
2 c_{36} & 2 c_{5}^{\prime}+2 s c_{36}^{\prime} \\
2 c_{5}^{\prime}+2 s c_{36}^{\prime} & 2 c_{3}^{\prime \prime}+2 s c_{5}^{\prime \prime}+s^{2} c_{36}^{\prime \prime}
\end{array}\right) .
$$

- $\quad \boldsymbol{X}_{\ell}^{h}$ at $p_{0}$ is $\mathcal{A}$-equivalent to the cuspidal cross cap if and only if $c_{36}=0, c_{1} c_{5} \neq 0$ and $c_{36}^{\prime} \neq 0$ hold at $p_{0}$.

Remark 8.7. Surfaces $\boldsymbol{X}_{\ell}^{h}$ satisfying $c_{3} \equiv 0$ be a horo-flat horo-cyclic surfaces which is investigated in [22]. Substituting $c_{3} \equiv 0$ in the formulas of Theorem 8.6, we have [22, Theorem $6.2]$.

Theorem 8.8. The singular set of $\boldsymbol{X}_{\ell}^{d}$ is $S\left(\boldsymbol{X}_{\ell}^{d}\right)=\left\{(s, t) \mid-2 c_{6}(t)-2 s c_{5}(t)+s^{2} c_{36}(t)=0\right\}$ and $\boldsymbol{X}_{\ell}^{d}$ is a frontal for any $\left(s_{0}, t_{0}\right) \in S\left(\boldsymbol{X}_{\ell}^{d}\right)$. Then we have the following assertions:

- If $c_{36}\left(t_{0}\right) \neq 0$ holds, then $\boldsymbol{X}_{\ell}^{d}$ to be a front near $p_{0}$.

- $\quad \boldsymbol{X}_{\ell}^{d}$ at $p_{0}$ is $\mathcal{A}$-equivalent to the cuspidal edge if and only if $c_{36} \neq 0$ and $\alpha_{l}^{d}:=2 c_{1}\left(c_{5}-\right.$ $\left.s c_{36}\right)+2 c_{6}^{\prime}+2 s c_{5}^{\prime}-s^{2} c_{36}^{\prime} \neq 0$ hold at $p_{0}$.

- $\quad \boldsymbol{X}_{\ell}^{d}$ at $p_{0}$ is $\mathcal{A}$-equivalent to swallowtail if and only if $c_{36} \neq 0, c_{5}-s c_{36} \neq 0, \alpha_{l}^{d}=0$ and $c_{1}\left(\alpha_{l}^{d}\right)_{s}+\left(\alpha_{l}^{d}\right)^{\prime} \neq 0$ hold at $p_{0}$.

- $\quad \boldsymbol{X}_{\ell}^{d}$ at $p_{0}$ is $\mathcal{A}$-equivalent to cuspidal butterfly if and only if $c_{36} \neq 0, c_{5}-s c_{36} \neq 0, \alpha_{l}^{d}=0$, $c_{1}\left(\alpha_{l}^{d}\right)_{s}+\left(\alpha_{l}^{d}\right)^{\prime}=0$ and $c_{1}^{2}\left(\alpha_{l}^{d}\right)_{s s}+2 c_{1}\left(\alpha_{l}^{d}\right)_{s}^{\prime}+c_{1}^{\prime}\left(\alpha_{l}^{d}\right)_{s}+\left(\alpha_{l}^{d}\right)^{\prime \prime} \neq 0$ hold at $p_{0}$. 
- $\quad \boldsymbol{X}_{\ell}^{d}$ at $p_{0}$ is $\mathcal{A}$-equivalent to the cuspidal lips (resp. cuspidal beaks) if and only if $c_{36} \neq$ $0,2 c_{6}^{\prime}+2 s c_{5}^{\prime}-s^{2} c_{36}^{\prime}=0, c_{5}-s c_{36}=0$ and $\operatorname{det} H_{\ell}^{d}>0$ (resp. $\operatorname{det} H_{\ell}^{d}<0$ and $\left.c_{1}\left(-2 c_{1} c_{36}+2 c_{5}^{\prime}-2 s c_{36}^{\prime}\right)+\left(2 c_{1}\left(c_{5}-s c_{36}\right)+2 c_{6}^{\prime}+2 s c_{5}^{\prime}-s^{2} c_{36}^{\prime}\right)^{\prime} \neq 0\right)$ hold at $p_{0}$, where

$$
H_{\ell}^{d}=\left(\begin{array}{cc}
-2 c_{36} & 2 c_{5}^{\prime}-2 s c_{36}^{\prime} \\
2 c_{5}^{\prime}-2 s c_{36}^{\prime} & 2 c_{6}^{\prime \prime}+2 s c_{5}^{\prime \prime}-s^{2} c_{36}^{\prime \prime}
\end{array}\right) .
$$

- $\quad \boldsymbol{X}_{\ell}^{d}$ at $p_{0}$ is $\mathcal{A}$-equivalent to the cuspidal cross cap if and only if $c_{36}=0, c_{1} c_{5} \neq 0$ and $c_{36}^{\prime} \neq 0$ hold at $p_{0}$.

Theorem 8.9. The singular set of $\boldsymbol{X}_{\ell}^{\ell}$ is $S\left(\boldsymbol{X}_{\ell}^{\ell}\right)=\left\{(s, t) \mid c_{3}(t)\left(s^{2}+1\right)+2 s c_{5}(t)+c_{6}(t)\left(s^{2}-1\right)=\right.$ $0\}$ and $\boldsymbol{X}_{\ell}^{\ell}$ is a frontal for any $\left(s_{0}, t_{0}\right) \in S\left(\boldsymbol{X}_{\ell}^{\ell}\right)$. Then we have the following assertions:

- If $c_{36}\left(t_{0}\right) \neq 0$ holds, then $\boldsymbol{X}_{\ell}^{\ell}$ to be a front near $p_{0}$.

- $\quad \boldsymbol{X}_{\ell}^{\ell}$ at $p_{0}$ is $\mathcal{A}$-equivalent to the cuspidal edge if and only if $c_{36} \neq 0$ and $\alpha_{l}^{l}:=-2 c_{1}\left(c_{5}+\right.$ $\left.s c_{36}\right)+c_{3}^{\prime}\left(s^{2}+1\right)+2 s c_{5}^{\prime}+c_{6}^{\prime}\left(s^{2}-1\right) \neq 0$ hold at $p_{0}$.

- $\quad \boldsymbol{X}_{\ell}^{\ell}$ at $p_{0}$ is $\mathcal{A}$-equivalent to the swallowtail if and only if $c_{36} \neq 0, c_{5}+s c_{36} \neq 0, \alpha_{l}^{\ell}=0$ and $-c_{1}\left(\alpha_{l}^{\ell}\right)_{s}\left(\alpha_{l}^{\ell}\right)^{\prime} \neq 0$ hold at $p_{0}$.

- $\quad \boldsymbol{X}_{\ell}^{\ell}$ at $p_{0}$ is $\mathcal{A}$-equivalent to the cuspidal butterfly if and only if $c_{36} \neq 0, c_{5}+s c_{36} \neq 0$, $\alpha_{\ell}^{\ell}=0,-c_{1}\left(\alpha_{\ell}^{\ell}\right)_{s}\left(\alpha_{\ell}^{\ell}\right)^{\prime}=0$ and $c_{1}^{2}\left(\alpha_{\ell}^{\ell}\right)_{s s}-2 c_{1}\left(\alpha_{\ell}^{\ell}\right)_{s}^{\prime}-c_{1}^{\prime}\left(\alpha_{\ell}^{\ell}\right)_{s}+\left(\alpha_{\ell}^{\ell}\right)^{\prime \prime} \neq 0$ hold at $p_{0}$.

- $\quad \boldsymbol{X}_{\ell}^{\ell}$ at $p_{0}$ is $\mathcal{A}$-equivalent to the cuspidal lips (resp. cuspidal beaks) if and only if $c_{36} \neq 0$, $c_{3}^{\prime}\left(s^{2}+1\right)+2 s c_{5}^{\prime}+c_{6}^{\prime}\left(s^{2}-1\right)=0, c_{5}+s c_{36}=0$ and $\operatorname{det} H_{\ell}^{\ell}>0\left(\right.$ resp. $\operatorname{det} H_{\ell}^{\ell}<0$ and $\left.-c_{1}\left(-2 c_{1} c_{36}+2\left(c_{5}+s c_{36}^{\prime}\right)\right)+\left(-2 c_{1}\left(c_{5}+s c_{36}\right)+c_{3}^{\prime}\left(s^{2}+1\right)+2 s c_{5}^{\prime}+c_{6}^{\prime}\left(s^{2}-1\right)\right)^{\prime} \neq 0\right)$ hold at $p_{0}$, where

$$
H_{\ell}^{\ell}=\left(\begin{array}{cc}
2 c_{36} & 2 c_{5}^{\prime}+2 s c_{36}^{\prime} \\
2 c_{5}^{\prime}+2 s c_{36}^{\prime} & c_{3}^{\prime \prime}\left(s^{2}+1\right)+2 s c_{5}^{\prime \prime}+c_{6}^{\prime \prime}\left(s^{2}-1\right)
\end{array}\right) .
$$

- $\quad \boldsymbol{X}_{\ell}^{\ell}$ at $p_{0}$ is $\mathcal{A}$-equivalent to the cuspidal cross cap if and only if $c_{36}=0, c_{1} c_{5} \neq 0$ and $c_{36}^{\prime} \neq 0$ hold at $p_{0}$.

\subsection{Singularities of dual surfaces of $a_{0}$}

In this section, we apply the criteria, and describe the conditions of singularities of dual surfaces of $\boldsymbol{a}_{0}$. In this section, we assume $c_{2} \equiv c_{3} \equiv 0$.

Theorem 8.10. The singular set of $\boldsymbol{X}_{h}^{d}$ is $S\left(\boldsymbol{X}_{h}^{d}\right)=\left\{(s, t) \mid c_{4}(t) \cos s+c_{5}(t) \sin s=0\right\}$ and $\boldsymbol{X}_{h}^{d}$ is a frontal for any $p_{0}=\left(s_{0}, t_{0}\right)$. Then we have the following assertions:

- If $c_{1} \neq 0$ holds, then $\boldsymbol{X}_{h}^{d}$ to be a front near $p_{0}$.

- $\quad \boldsymbol{X}_{h}^{d}$ at $p_{0}$ is $\mathcal{A}$-equivalent to the cuspidal edge if and only if $c_{1} \neq 0$ and $\alpha_{h}^{d}:=-c_{6}\left(c_{4} \sin s-\right.$ $\left.c_{5} \cos s\right)-\left(c_{4}^{\prime} \cos s+c_{5}^{\prime} \sin s\right) \neq 0$ holds at $p_{0}$.

- $\boldsymbol{X}_{h}^{d}$ at $p_{0}$ is $\mathcal{A}$-equivalent to the swallowtail if and only if $c_{1} \neq 0,-c_{4} \sin s+c_{5} \cos s \neq 0$, $\alpha_{h}^{d}=0$ and $-c_{6}\left(\alpha_{h}^{d}\right)_{s}+\left(\alpha_{h}^{d}\right)^{\prime} \neq 0$.

- $\quad \boldsymbol{X}_{h}^{d}$ at $p_{0}$ is $\mathcal{A}$-equivalent to the cuspidal butterfly if and only if $c_{1} \neq 0,-c_{4} \sin s+$ $c_{5} \cos s \neq 0, \alpha_{h}^{d}=0,-c_{6}\left(\alpha_{h}^{d}\right)_{s}+\left(\alpha_{h}^{d}\right)^{\prime}=0$ and $c_{6}^{2}\left(\alpha_{h}^{d}\right)_{s s}-2 c_{6}\left(\alpha_{h}^{d}\right)_{s}^{\prime}-c_{6}^{\prime}\left(\alpha_{h}^{d}\right)_{s}+\left(\alpha_{h}^{d}\right)^{\prime \prime} \neq 0$ holds at $p_{0}$.

- $\boldsymbol{X}_{h}^{d}$ at $p_{0}$ is $\mathcal{A}$-equivalent to the cuspidal lips (resp. cuspidal beaks) if and only if $c_{1} \neq 0$, $c_{4}^{\prime} \cos s+c_{5}^{\prime} \sin s=0, c_{4} \sin s-c_{5} \cos s=0$, and $\operatorname{det} H_{h}^{d}>0$ (resp. $\operatorname{det} H_{h}^{d}<0$ and $\left.-c_{6}\left(\alpha_{h}^{d}\right)_{s}+\left(\alpha_{h}^{d}\right)^{\prime} \neq 0\right)$ hold at $p_{0}$, where

$$
H_{h}^{d}=\left(\begin{array}{cc}
-c_{4} \cos s-c_{5} \sin s & c_{4}^{\prime} \sin s-c_{5}^{\prime} \cos s \\
c_{4}^{\prime} \sin s-c_{5}^{\prime} \cos s & c_{4}^{\prime \prime} \cos s+c_{5}^{\prime \prime} \sin s
\end{array}\right)
$$


- $\quad \boldsymbol{X}_{h}^{d}$ at $p_{0}$ is $\mathcal{A}$-equivalent to the cuspidal cross cap if and only if $c_{1}=0, c_{5} c_{6} \neq 0$ and $c_{1}^{\prime} \neq 0$ hold at $p_{0}$.

Theorem 8.11. The singular set of $\boldsymbol{X}_{h}^{\ell}$ is $S\left(\boldsymbol{X}_{h}^{\ell}\right)=\left\{(s, t) \mid c_{1}(t)-c_{4}(t) \cos s-c_{5}(t) \sin s=0\right\}$ and $\boldsymbol{X}_{h}^{\ell}$ is a frontal for any $p_{0}=\left(s_{0}, t_{0}\right)$. Then we have the following assertions:

- If $c_{1}\left(t_{0}\right) \neq 0$ holds, then $\boldsymbol{X}_{h}^{\ell}$ to be a front near $p_{0}$.

- $\quad \boldsymbol{X}_{h}^{\ell}$ at $p_{0}$ is $\mathcal{A}$-equivalent to the cuspidal edge if and only if $c_{1} \neq 0$ and $\alpha_{h}^{\ell}:=-c_{6}\left(c_{4} \sin s-\right.$ $\left.c_{5} \cos s\right)+c_{1}^{\prime}-c_{4}^{\prime} \cos s-c_{5}^{\prime} \sin s \neq 0$ holds at $p_{0}$.

- $\boldsymbol{X}_{h}^{\ell}$ at $p_{0}$ is $\mathcal{A}$-equivalent to the swallowtail if and only if $c_{1} \neq 0, c_{4} \sin s-c_{5} \cos s \neq 0$, $\alpha_{h}^{\ell}=0$ and $-c_{6}\left(\alpha_{h}^{\ell}\right)_{s}+\left(\alpha_{h}^{\ell}\right)^{\prime} \neq 0$ holds at $p_{0}$.

- $\quad \boldsymbol{X}_{h}^{\ell}$ at $p_{0}$ is $\mathcal{A}$-equivalent to the cuspidal butterfly if and only if $c_{1} \neq 0, c_{4} \sin s-c_{5} \cos s \neq$ $0, \alpha_{h}^{\ell}=0$ and $-c_{6}\left(\alpha_{h}^{\ell}\right)_{s}+\left(\alpha_{h}^{\ell}\right)^{\prime}=0$ and $c_{6}^{2}\left(\alpha_{h}^{\ell}\right)_{s s}-2 c_{6}\left(\alpha_{h}^{\ell}\right)_{s}^{\prime}-c_{6}^{\prime}\left(\alpha_{h}^{\ell}\right)_{s}+\left(\alpha_{h}^{\ell}\right)^{\prime \prime} \neq 0$ holds at $p_{0}$.

- $\quad \boldsymbol{X}_{h}^{\ell}$ at $p_{0}$ is $\mathcal{A}$-equivalent to the cuspidal lips (resp. cuspidal beaks) if and only if $c_{1} \neq 0$, $c_{4} \sin s-c_{5} \cos s=0, c_{1}^{\prime}-c_{4}^{\prime} \cos s-c_{5}^{\prime} \sin s=0$ and $\operatorname{det} H_{h}^{\ell}>0$ (resp. $\operatorname{det} H_{h}^{\ell}$ and $\left.-c_{6}\left(\alpha_{h}^{\ell}\right)_{s}+\left(\alpha_{h}^{\ell}\right)^{\prime} \neq 0\right)$ holds at $p_{0}$, where

$$
H_{h}^{\ell}=\left(\begin{array}{cc}
c_{4} \cos s+c_{5} \sin s & c_{4}^{\prime} \sin s-c_{5}^{\prime} \cos s \\
c_{4}^{\prime} \sin s-c_{5}^{\prime} \cos s & c_{1}^{\prime \prime}-c_{4}^{\prime \prime} \cos s-c_{5}^{\prime \prime} \sin s
\end{array}\right)
$$

- $\quad \boldsymbol{X}_{h}^{\ell}$ at $p_{0}$ is $\mathcal{A}$-equivalent to the cuspidal cross cap if and only if $c_{1}=0, c_{5} c_{6} \neq 0$ and $c_{1}^{\prime} \neq 0$ hold at $p_{0}$.

Remark 8.12. Surfaces $\boldsymbol{X}_{h}^{\ell}$ satisfying $c_{6} \equiv 0$ is called a hyperbolic-flat tangent lightcone circular surface which is investigated in [22]. Substituting $c_{6} \equiv 0$ in the formulas of Theorem 8.11, we have [22, Theorem 8.2].

\subsection{Singularities of dual surfaces of $a_{2}$}

In this section, we apply criteria and describe the conditions of singularities of dual surfaces of $\boldsymbol{a}_{2}$. In this section, we assume that $c_{2} \equiv c_{6} \equiv 0$.

Theorem 8.13. The singular set of $\boldsymbol{X}_{d}^{h}$ is $S\left(\boldsymbol{X}_{d}^{h}\right)=\left\{(s, t) \mid c_{1}(t) \cosh s-c_{5}(t) \sinh s=0\right\}$ and $\boldsymbol{X}_{d}^{h}$ is a frontal for any $p_{0}=\left(s_{0}, t_{0}\right)$. Then we have the following assertions:

- If $c_{4} \neq 0$ holds, then $\boldsymbol{X}_{d}^{h}$ ia a front near $p_{0}$.

- $\quad \boldsymbol{X}_{d}^{h}$ at $p_{0}$ is $\mathcal{A}$-equivalent to the cuspidal edge if and only if $c_{4} \neq 0$ and $\alpha_{d}^{h}:=-c_{3}\left(c_{1} \sinh s-\right.$ $\left.c_{5} \cosh s\right)+c_{1}^{\prime} \cosh s-c_{5}^{\prime} \sinh s \neq 0$ hold at $p_{0}$.

- $\quad \boldsymbol{X}_{d}^{h}$ at $p_{0}$ is $\mathcal{A}$-equivalent to the swallowtail if and only if $c_{4} \neq 0, c_{1} \sinh s-c_{5} \cosh s \neq 0$ and $\alpha_{d}^{h}=0$ and $-c_{3}\left(\alpha_{d}^{h}\right)_{s}+\left(\alpha_{d}^{h}\right)^{\prime} \neq 0$ hold at $p_{0}$.

- $\quad \boldsymbol{X}_{d}^{h}$ at $p_{0}$ is $\mathcal{A}$-equivalent to the cuspidal butterfly if and only if $c_{4} \neq 0, c_{1} \sinh s-$ $c_{5} \cosh s \neq 0$ and $\alpha_{d}^{h}=-c_{3}\left(\alpha_{d}^{h}\right)_{s}+\left(\alpha_{d}^{h}\right)^{\prime}=0,-c_{3}\left(\alpha_{d}^{h}\right)_{s}+\left(\alpha_{d}^{h}\right)^{\prime}=0$ and $c_{3}^{2}\left(\alpha_{d}^{h}\right)_{s s}-$ $2 c_{3}\left(\alpha_{d}^{h}\right)_{s}^{\prime}-c_{3}^{\prime}\left(\alpha_{d}^{h}\right)_{s}+\left(\alpha_{d}^{h}\right)^{\prime \prime} \neq 0$ hold at $p_{0}$.

- $\quad \boldsymbol{X}_{d}^{h}$ at $p_{0}$ is $\mathcal{A}$-equivalent to the cuspidal lips (resp. cuspidal beaks) if and only if $c_{4} \neq 0$, $c_{1} \sinh s-c_{5} \cosh s=0, c_{1}^{\prime} \cosh s-c_{5}^{\prime} \sinh s=0 \operatorname{det} H_{d}^{h}>0$ (resp. $\operatorname{det} H_{d}^{h}<0$ and $\left.-c_{3}\left(\alpha_{d}^{h}\right)_{s}+\left(\alpha_{d}^{h}\right)^{\prime} \neq 0\right)$ hold at $p_{0}$, where

$$
H_{d}^{h}=\left(\begin{array}{ll}
c_{1} \cosh s-c_{5} \sinh s & c_{1}^{\prime} \sinh s-c_{5}^{\prime} \cosh s \\
c_{1}^{\prime} \sinh s-c_{5}^{\prime} \cosh s & c_{1}^{\prime \prime} \cosh s-c_{5}^{\prime \prime} \sinh s
\end{array}\right)
$$


- $\quad \boldsymbol{X}_{d}^{h}$ at $p_{0}$ is $\mathcal{A}$-equivalent to the cuspidal cross cap if and only if $c_{4}=0, c_{3} c_{5} \neq 0$ and $c_{4}^{\prime} \neq 0$ hold at $p_{0}$.

Theorem 8.14. The singular set of $\boldsymbol{X}_{d}^{\ell}$ is $S\left(\boldsymbol{X}_{d}^{\ell}\right)=\left\{(s, t) \mid-c_{4}(t)+c_{1}(t) \cosh s-c_{5}(t) \sinh s=\right.$ $0\}$ and $\boldsymbol{X}_{d}^{\ell}$ is a frontal for any $p_{0}=\left(s_{0}, t_{0}\right) \in S\left(\boldsymbol{X}_{d}^{\ell}\right)$. Then we have following assertions:

- If $c_{4} \neq 0$ holds, then $\boldsymbol{X}_{d}^{\ell}$ is a front near $p_{0}$.

- $\quad \boldsymbol{X}_{d}^{\ell}$ at $p_{0}$ is $\mathcal{A}$-equivalent to the cuspidal edge if and only if $c_{4} \neq 0$ and $\alpha_{d}^{\ell}:=-c_{3}\left(c_{1} \sinh s-\right.$ $\left.c_{5} \cosh s\right)-c_{4}^{\prime}+c_{1}^{\prime} \cosh s-c_{5}^{\prime} \sinh s \neq 0$ hold at $p_{0}$.

- $\quad \boldsymbol{X}_{d}^{\ell}$ at $p_{0}$ is $\mathcal{A}$-equivalent to the swallowtail if and only if $c_{4} \neq 0, c_{1} \sinh s-c_{5} \cosh s \neq 0$, $\alpha_{d}^{\ell}=0$ and $-c_{3}\left(\alpha_{d}^{\ell}\right)_{s}+\left(\alpha_{d}^{\ell}\right)^{\prime} \neq 0$ hold at $p_{0}$.

- $\quad \boldsymbol{X}_{d}^{\ell}$ at $p_{0}$ is $\mathcal{A}$-equivalent to the cuspidal butterfly if and only if $c_{4} \neq 0, c_{1} \sinh s-$ $c_{5} \cosh s \neq 0 c_{1} \sinh s-c_{5} \cosh s \neq 0, \alpha_{d}^{\ell}=0-c_{3}\left(\alpha_{d}^{\ell}\right)_{s}+\left(\alpha_{d}^{\ell}\right)^{\prime}=0$ and $c_{3}^{2}\left(\alpha_{d}^{\ell}\right)_{s s}-$ $2 c_{3}\left(\alpha_{d}^{\ell}\right)_{s}^{\prime}-c_{3}^{\prime}\left(\alpha_{d}^{\ell}\right)_{s}+\left(\alpha_{d}^{\ell}\right)^{\prime \prime} \neq 0$ holds at $p_{0}$.

- $\quad \boldsymbol{X}_{d}^{\ell}$ at $p_{0}$ is $\mathcal{A}$-equivalent to the cuspidal lips (resp. cuspidal beaks) $c_{4} \neq 0, c_{1} \sinh s-$ $c_{5} \cosh s=0,-c_{4}^{\prime}+c_{1}^{\prime} \cosh s-c_{5}^{\prime} \sinh s=0$ and $\operatorname{det} H_{d}^{\ell}>0$ (resp. $\operatorname{det} H_{d}^{\ell}<0$ and $\left.-c_{3}\left(\alpha_{d}^{\ell}\right)_{s}+\left(\alpha_{d}^{\ell}\right)^{\prime} \neq 0\right)$ hold at $p_{0}$, where

$$
H_{d}^{\ell}=\left(\begin{array}{cc}
c_{1} \cosh s-c_{5} \sinh s & c_{1}^{\prime} \sinh s-c_{5}^{\prime} \cosh s \\
c_{1}^{\prime} \sinh s-c_{5}^{\prime} \cosh s & -c_{4}^{\prime \prime}+c_{1}^{\prime \prime} \cosh s-c_{5}^{\prime \prime} \sinh s
\end{array}\right) .
$$

- $\quad \boldsymbol{X}_{d}^{\ell}$ at $p_{0}$ is $\mathcal{A}$-equivalent to the cuspidal cross cap if and only if $c_{4}=0, c_{3} c_{5} \neq 0$ and $c_{4}^{\prime} \neq 0$ hold at $p_{0}$.

We shall give proofs of these theorems in the following.

Proof of Theorem 8.9. Since

$$
\begin{aligned}
\left(\boldsymbol{X}_{\ell}^{\ell}\right)_{s,} & =2 s \boldsymbol{a}_{0}+2 \boldsymbol{a}_{1}+2 s \boldsymbol{a}_{2} \\
\left(\boldsymbol{X}_{\ell}^{\ell}\right)^{\prime} & =2 s c_{1} \boldsymbol{a}_{0}+2 c_{1} \boldsymbol{a}_{1}+2 c_{1} \boldsymbol{a}_{2}+\left(c_{3}\left(s^{2}+1\right)+2 s c_{5}+c_{6}\left(s^{2}-1\right)\right) \boldsymbol{a}_{3},
\end{aligned}
$$

it follows that $S\left(\boldsymbol{X}_{\ell}^{\ell}\right)=\left\{(s, t) \mid c_{3}(t)\left(s^{2}+1\right)+2 s c_{5}(t)+c_{6}(t)\left(s^{2}-1\right)=0\right\}$. Furthermore, an isotropic map $\left(\boldsymbol{X}_{\ell}^{\ell}, \ell\right): U \rightarrow \Delta_{4}$ is a Legendrian immersion if and only if $c_{36} \neq 0$ on $S\left(\boldsymbol{X}_{\ell}^{\ell}\right)$. In this case $\boldsymbol{X}_{\ell}^{\ell}$ is a front nearp $p_{0}$. Since $\boldsymbol{a}_{0}$ and $\boldsymbol{a}_{2}$ are linearly independent to $T L C^{*}$, we can choose the discriminant function $\lambda$ as

$$
\lambda=\operatorname{det}\left(\left(\boldsymbol{X}_{\ell}^{\ell}\right)_{s},\left(\boldsymbol{X}_{\ell}^{\ell}\right)^{\prime}, \boldsymbol{a}_{0}, \boldsymbol{a}_{2}\right)=-2\left(\left(s^{2}+1\right) c_{3}(t)+2 c_{5}(t)+2 s c_{6}(t)\right) .
$$

Since the kernel direction of $d \boldsymbol{X}_{\ell}^{\ell}$ on singular set is $\eta=-c_{1} \partial s+\partial t$ and we can take a transversal vector field $\partial s$, we have

$$
\begin{aligned}
\eta \lambda & =-2 c_{1}\left(c_{5}+s c_{36}\right)+c_{3}^{\prime}\left(s^{2}+1\right)+2 s c_{5}^{\prime}+c_{6}^{\prime}\left(s^{2}-1\right) \\
\eta \eta \lambda & =-c_{1}\left(-2 c_{1} c_{36}+2\left(c_{5}+s c_{36}^{\prime}\right)\right) \\
\text { Hess } \lambda= & \left.\begin{array}{cc}
2 c_{36} & \left.+2 c_{1}\left(c_{5}+s c_{36}\right)+c_{3}^{\prime}\left(s^{2}+1\right)+2 s c_{5}^{\prime}+c_{6}^{\prime}\left(s^{2}-1\right)\right)^{\prime} \\
2 c_{5}^{\prime}+2 s c_{36}^{\prime} & c_{3}^{\prime \prime}\left(s^{2}+1\right)+2 s c_{5}^{\prime \prime}+c_{6}^{\prime \prime}\left(s^{2}-1\right)
\end{array}\right)
\end{aligned}
$$

Hence we have all assertions of Theorem 8.9 except the case for the condition of cuspidal cross caps. We give the proof of the condition for cuspidal cross cap as follows: Let us define a lift $\omega: U \rightarrow T^{*} L C^{*}$ by

$$
\omega_{p}(v)=\langle v, \ell(p)\rangle, v \in T_{X_{\ell}^{\ell}(p)} L C^{*}, p \in U
$$


Then $\omega$ does not have intersection with the zero section. Since $(\pi \circ \omega)_{*}(z)=d \boldsymbol{X}_{\ell}^{\ell}(z)$ for any vector $z \in T_{p} U$, we have $\left\langle\boldsymbol{\ell}, d \boldsymbol{X}_{\ell}^{\ell}\right\rangle=\theta_{4}\left(\boldsymbol{a}_{3}, \boldsymbol{X}_{\ell}^{\ell}\right)=0$. Thus we have $(\pi \circ \omega)_{*}\left(T_{p} U\right) \subset \operatorname{ker} \omega_{p}$. This means that $\omega$ is the admissible lift of $\boldsymbol{X}_{\ell}^{\ell}$. Under the assumption that $c_{36}\left(t_{0}\right)=0, \lambda_{s}\left(s_{0}, t_{0}\right) \neq 0$ if and only if $c_{5}\left(t_{0}\right) \neq 0$. Then $S\left(\boldsymbol{X}_{\ell}^{\ell}\right)$ can be parameterized as $(s(t), t)$ for some function $s(t)$. Putting $\xi(t)=\boldsymbol{a}_{3}(t)$, then $\xi$ is a non-zero vector field along $\left.\boldsymbol{X}_{\ell}^{\ell}\right|_{S\left(X_{\ell}^{\ell}\right)}$. Since $\left\langle\xi, \boldsymbol{X}_{\ell}^{\ell}\right\rangle=0$, vector field $\xi$ satisfies the conditions of Theorem 8.5. Therefore the function $\psi_{X_{\ell}^{\ell}}(t)$ is equal to $\langle\eta \xi, \ell\rangle(t)=-c_{36}(t)$. On the other hand, if $\lambda_{s}\left(s_{0}, t_{0}\right)=0$ and $\lambda^{\prime}\left(s_{0}, t_{0}\right) \neq 0$, then $\left(\boldsymbol{X}_{\ell}^{\ell},\left(s_{0}, t_{0}\right)\right)$ is not $\mathcal{A}$-equivalent to the cuspidal cross cap. This competes the proof of Theorem 8.9.

We can give the proofs of Theorems $8.6,8.8,8.10,8.11,8.13$ and 8.14 by the same arguments as those of the above proof. We only state the fundamental data here, and omit the detailed proof. The discriminant function $\lambda$, null vector field $\eta$, the one-form $\omega$ and the vector field $\xi$ for each dual surfaces are shown in the Table 1.

\begin{tabular}{|c|c|c|c|c|}
\hline Surface & $\lambda$ & $\eta$ & $\omega$ & $\xi$ \\
\hline \hline $\boldsymbol{X}_{\ell}^{h}$ & $2 c_{3}+2 s c_{5}+s^{2} c_{36}$ & $\left(-c_{1}, 1\right)$ & $\langle *, \boldsymbol{\ell}\rangle$ & $\boldsymbol{a}_{3}$ \\
\hline $\boldsymbol{X}_{\ell}^{d}$ & $-2 c_{6}-2 s c_{5}+s^{2} c_{36}$ & $\left(c_{1}, 1\right)$ & $\langle *, \boldsymbol{\ell}\rangle$ & $\boldsymbol{a}_{3}$ \\
\hline $\boldsymbol{X}_{h}^{d}$ & $c_{4} \cos s+c_{5} \sin s$ & $\left(-c_{6}, 1\right)$ & $\left\langle *, \boldsymbol{a}_{0}\right\rangle$ & $\boldsymbol{a}_{1}$ \\
\hline $\boldsymbol{X}_{h}^{\ell}$ & $c_{1}-c_{4} \cos s-c_{5} \sin s$ & $\left(-c_{6}, 1\right)$ & $\left\langle *, \boldsymbol{a}_{0}\right\rangle$ & $\boldsymbol{a}_{1}$ \\
\hline $\boldsymbol{X}_{d}^{h}$ & $c_{1} \cosh s-c_{5} \sinh s$ & $\left(-c_{3}, 1\right)$ & $\left\langle *, \boldsymbol{a}_{2}\right\rangle$ & $\boldsymbol{a}_{1}$ \\
\hline $\boldsymbol{X}_{d}^{\ell}$ & $-c_{4}+c_{1} \cosh s-c_{5} \sinh s$ & $\left(-c_{3}, 1\right)$ & $\left\langle *, \boldsymbol{a}_{2}\right\rangle$ & $\boldsymbol{a}_{1}$ \\
\hline
\end{tabular}

Table 1: Fundamental data to recognize the conditions of singularities of dual surfaces

\section{Dualities of singularities}

Comparing Theorems 8.6, 8.8 and 8.9, when singular point is always $(0, t)$, with Theorems 8.10, $8.11,8.13$ and 8.14 we observe a certain duality between swallowtails and cuspidal cross caps. It can be summerized as follows.

Remark 9.1. The conditions that singular set is equal to the curve $(0, t)$ is

- $c_{3} \equiv 0$ for $\boldsymbol{X}_{\ell}^{h}$,

- $c_{6} \equiv 0$ for $\boldsymbol{X}_{\ell}^{d}$,

- $c_{3}-c_{6} \equiv 0$ for $\boldsymbol{X}_{\ell}^{\ell}$

- $c_{4} \equiv 0$ for $\boldsymbol{X}_{h}^{d}$

- $c_{1}-c_{4} \equiv 0$ for $\boldsymbol{X}_{h}^{\ell}$,

- $c_{1} \equiv 0$ for $\boldsymbol{X}_{d}^{h}$,

- $\quad c_{1}-c_{4} \equiv 0$ for $\boldsymbol{X}_{d}^{\ell}$.

Moreover, if $c_{2} \equiv c_{3} \equiv c_{1}-c_{4} \equiv 0$ for any $t$, then $\boldsymbol{X}_{\ell}^{h}$ at $\left(0, t_{0}\right)$ is $\mathcal{A}$-equivalent to the swallowtail if and only if $c_{6}=0$ and $c_{1} c_{6}^{\prime} \neq 0$ at $t_{0}$. This condition is the same as $\boldsymbol{X}_{h}^{\ell}$ at $\left(0, t_{0}\right)$ is $\mathcal{A}$-equivalent to the cuspidal cross cap. Furthermore, $\boldsymbol{X}_{\ell}^{h}$ at $\left(0, t_{0}\right)$ is $\mathcal{A}$-equivalent to the cuspidal cross cap if and only if $c_{1}=0$ and $c_{6} c_{1}^{\prime} \neq 0$ at $t_{0}$. This condition is the same as $\boldsymbol{X}_{h}^{\ell}$ at $\left(0, t_{0}\right)$ is $\mathcal{A}$-equivalent to the swallowtail. Like as this argument, we have the same type condition of singular points for dual surfaces when the singular set is equal to $(0, t)$.

We can summerize this situation on the Table 2 . In the table, $S$ means the singular set. 


\begin{tabular}{|l||l||l||l||l||l|}
\hline & duality & $S=\{(0, t)\}$ & cuspidal edge & swallowtail & cuspidal cross cap \\
\hline $\boldsymbol{X}_{\ell}^{h}$ & $c_{2} \equiv 0$ & $c_{3} \equiv 0$ & $c_{6} \neq 0$, & $c_{6} c_{5} \neq 0$, & $c_{1} c_{5} \neq 0,, c_{6}^{\prime} \neq 0$, \\
& $c_{1}-c_{4} \equiv 0$ & & $c_{1} c_{5} \neq 0$ & $c_{1}=0, c_{1}^{\prime} \neq 0$, & $c_{6}=0, c_{6}^{\prime}$ \\
\hline $\boldsymbol{X}_{\ell}^{d}$ & $c_{2} \equiv 0$ & $c_{6} \equiv 0$ & $c_{3} \neq 0$, & $c_{3} c_{5} \neq 0$, & $c_{1} c_{5} \neq 0$, \\
& $c_{1}-c_{4} \equiv 0$ & & $c_{1} c_{5} \neq 0$ & $c_{1}=0, c_{1}^{\prime} \neq 0$ & $c_{3}=0, c_{3}^{\prime} \neq 0$, \\
\hline $\boldsymbol{X}_{\ell}^{\ell}$ & $c_{2} \equiv 0$ & $c_{3}-c_{6} \equiv 0$ & $c_{36} \neq 0$, & $c_{36} c_{5} \neq 0$, & $c_{1} c_{5} \neq 0$, \\
& $c_{1}-c_{4} \equiv 0$ & & $c_{1} c_{5} \neq 0$ & $c_{1}=0, c_{1}^{\prime} \neq 0$ & $c_{36}=0, c_{36}^{\prime} \neq 0$, \\
\hline \hline $\boldsymbol{X}_{h}^{d}$ & $c_{2} \equiv 0$ & $c_{4} \equiv 0$ & $c_{1} \neq 0$ & $c_{1} c_{5} \neq 0$ & $c_{5} c_{6} \neq 0$ \\
& $c_{3} \equiv 0$ & & $c_{5} c_{6} \neq 0$ & $c_{6}=0, c_{6}^{\prime} \neq 0$ & $c_{1}=0, c_{1}^{\prime} \neq 0$ \\
\hline $\boldsymbol{X}_{h}^{\ell}$ & $c_{2} \equiv 0$ & $c_{1}-c_{4} \equiv 0$ & $c_{1} \neq 0$ & $c_{1} c_{5} \neq 0$ & $c_{5} c_{6} \neq 0$ \\
& $c_{3} \equiv 0$ & & $c_{5} c_{6} \neq 0$ & $c_{6}=0, c_{6}^{\prime} \neq 0$ & $c_{1}=0, c_{1}^{\prime} \neq 0$ \\
\hline \hline $\boldsymbol{X}_{d}^{h}$ & $c_{2} \equiv 0$ & $c_{1} \equiv 0$ & $c_{4} \neq 0$ & $c_{4} c_{5} \neq 0$ & $c_{3} c_{5} \neq 0$ \\
& $c_{6} \equiv 0$ & & $c_{3} c_{5} \neq 0$ & $c_{3}=0, c_{3}^{\prime} \neq 0$ & $c_{4}=0, c_{4}^{\prime} \neq 0$ \\
\hline $\boldsymbol{X}_{d}^{\ell}$ & $c_{2} \equiv 0$ & $c_{1}-c_{4} \equiv 0$ & $c_{4} \neq 0$ & $c_{4} c_{5} \neq 0$ & $c_{3} c_{5} \neq 0$ \\
& $c_{6} \equiv 0$ & & $c_{3} c_{5} \neq 0$ & $c_{3}=0, c_{3}^{\prime} \neq 0$ & $c_{4}=0, c_{4}^{\prime} \neq 0$ \\
\hline
\end{tabular}

Table 2: Dualities of condition for singularity.

We can observe there are some dual relations of conditions for singularities of the swallowtail and the cuspidal cross cap on each dual points of surfaces. Furthermore, the condition of holding the duality and that the singular set is $\{(0, t)\}$ are the same between $\boldsymbol{X}_{\ell}^{h}$ and $\boldsymbol{X}_{h}^{\ell}$ (resp. between $\boldsymbol{X}_{\ell}^{d}$ and $\left.\boldsymbol{X}_{d}^{\ell}\right)$ :

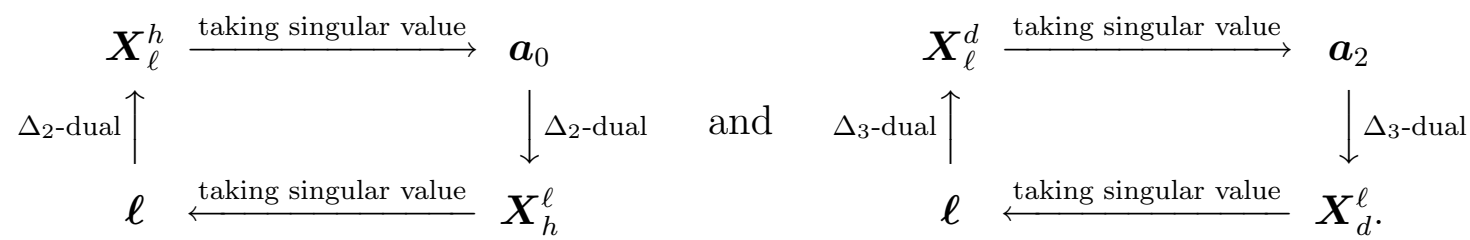

Like as the remark, a duality between swallowtail and cuspidal cross cap have been pointed out in many researches, for example, [33, 13, 22]. In this section, we give an interpretation for this duality. Firstly, we prove the following lemma.

Lemma 9.2. Let $M_{i}(i=1,2)$ are three dimensional manifolds and $\Delta \subset M_{1} \times M_{2}$ a five dimensional submanifold with the contact structure. Assume that the canonical projection $\pi_{1}$ : $\Delta \rightarrow M_{1}$ is a Legendre fibrations. If an isotropic map $L_{1}=\left(f_{1}, \nu_{1}\right)$ and a frontal $f_{2}: U \rightarrow M_{2}$ satisfies that $p$ is a non-degenerate singular point of both $f_{i}(i=1,2)$ and $\nu_{1}$ degenerates a curve such that $\nu_{1}=f_{2} \circ \sigma$, where $\sigma$ is a submersion $U \rightarrow S(f)$. If the null direction of $f_{1}$ does not parallel to the kernel of $\sigma$, then the following two conditions are equivalent.

- $\quad L_{1}$ is a Legendrian immersion.

- $\quad$ The null direction of $f_{2}$ at $p$ is transversal to $S\left(f_{1}\right)$.

Proof. Since $p$ is a non-degenerate singular point, $L_{1}$ is a Legendrian immersion if and only if the directional derivative $\eta_{1} \nu_{1}$ does not vanish. this is equivalent to $d f_{2}\left(\eta_{1}\right)(\sigma)$ does not vanish. This is equivalent to that the tangential direction of $S\left(f_{1}\right)$ does not parallel to $\eta_{2}$. This is equivalent to $\eta_{2}$ is transversal to $S\left(f_{1}\right)$. This completes the proof.

Theorem 9.3. Let $p$ is a non-degenerate singular point of a frontal $f$. Then we have the following criteria of singularities using the function $\psi_{f}$ defined in (8.2). 
(1) If $\psi_{f}(p) \neq 0$ then $f$ at $p$ is $\mathcal{A}$-equivalent to the cuspidal edge.

(2) Assume that $f$ is a front. If $\psi_{f}(p)=0$ and $(d / d t) \psi_{f}(p) \neq 0$, then $f$ at $p$ is $\mathcal{A}$-equivalent to the swallowtail.

(3) Assume that the null direction at $p$ is transversal to $S(f)$. If $\psi_{f}(p)=0$ and $(d / d t) \psi_{f}(p) \neq$ 0 , then $f$ at $p$ is $\mathcal{A}$-equivalent to the cuspidal cross cap.

Proof. Since the conditions are independent of the choice of coordinates, we take the coordinate system $(u, v)$ satisfying $S(f)=\{v=0\}$. Under this conditions, $\psi_{f}$ is proportional to $\phi_{f}$, where $\phi_{f}$ is defined in (8.1). Firstly, we prove (1). The condition $\phi_{f} \neq 0$ implies that $f_{u}$ and $\eta \nu$ are linearly independent. Since $\nu$ points the kernel direction of $d f$, this implies that $f$ to be a front. Moreover, we have $f_{u} \neq \mathbf{0}$, this implies that $\eta$ does not tangent to $S(f)$. By Theorem 8.1, we have (1).

Next, we assume that $f$ to be a front and $\phi_{f}=0$ at $p$. Then this condition implies $f_{u}(p)=\mathbf{0}$, namely, $\eta$ tangents $S(f)$ at $p$. Thus we can take a function $\beta(u)$ such that $\eta(u)=$ $\partial / \partial u+\beta(u) \partial / \partial v, \beta(0)=0$. By Theorem 8.1, $f$ at $p$ is $\mathcal{A}$-equivalent to the swallowtail if and only if $\beta^{\prime}(0) \neq 0$. On the other hand, $(d / d t) \phi_{f}(p) \neq 0$ implies that $\operatorname{det}\left(f_{u u}, \nu, \nu_{u}\right)(p) \neq 0$. Since $f_{v}, \nu$ and $\nu_{u}$ are linear independent at $p$, this is equivalent to $\left\langle f_{u u}, f_{v}\right\rangle(p) \neq 0$. Since $\eta$ is the null vector field on the $u$-axis, $f_{u}+\beta(u)+f_{v}=\mathbf{0}$ holds on the $u$-axis. Thus $\left\langle f_{u u}, f_{v}\right\rangle(p) \neq 0$ implies $\beta^{\prime}(0) \neq 0$. This completes the proof. (3) is directly holds from Theorem 8.1.

We can give the alternative proof of Theorem 8.11 in the special case of $c_{1}-c_{4} \equiv 0$ and $c_{5} \neq 0$.

Proof of Theorem 8.11 If $c_{2} \equiv c_{3} \equiv c_{1}-c_{4} \equiv 0$, and $c_{5}\left(t_{0}\right) \neq 0$, then by Theorem $8.6 \boldsymbol{X}_{h}^{\ell}$ at $\left(t_{0}, 0\right)$ is $\mathcal{A}$-equivalent to the swallowtail if and only if $c_{6} \neq 0, c_{1}=0$ and $c_{1}^{\prime} \neq 0$ at $t_{0}$. Furthermore, $\boldsymbol{X}_{h}^{\ell}$ at $\left(t_{0}, 0\right)$ is $\mathcal{A}$-equivalent to the cuspidal cross cap if and only if $c_{1} \neq 0, c_{6}=0$ and $c_{6}^{\prime} \neq 0$ at $t_{0}$.

Under the assumptions $c_{2} \equiv c_{3} \equiv c_{1}-c_{4} \equiv 0$ and $c_{5}\left(t_{0}\right) \neq 0$, it holds that $S\left(\boldsymbol{X}_{\ell}^{h}\right)=$ $S\left(\boldsymbol{X}_{h}^{\ell}\right)=\{(t, 0)\}$ near $\left(t_{0}, 0\right)$. Hence we can apply Lemma 9.2 and Theorem 9.3. This means that the condition for swallowtail and cuspidal cross cap of the dual surface are obtained by only interchangeing the condition for cuspidal cross cap and swallowtail of the original surface. Thus we have that $\boldsymbol{X}_{h}^{\ell}$ at $\left(t_{0}, 0\right)$ is $\mathcal{A}$-equivalent to the cuspidal cross cap if and only if $c_{6} \neq 0$, $c_{1}=0$ and $c_{1}^{\prime} \neq 0$ at $t_{0}$. Furthermore, $\boldsymbol{X}_{h}^{\ell}$ at $\left(t_{0}, 0\right)$ is $\mathcal{A}$-equivalent to the capswallowtail if and only if $c_{1} \neq 0, c_{6}=0$ and $c_{6}^{\prime} \neq 0$ at $t_{0}$. This is the same as Theorem 8.11 under the assumption $c_{1}-c_{4} \equiv 0$.

\section{A A criterion for the cuspidal butterfly}

In this section, we give a proof of Theorem 8.2. The main tool to prove it is the notion of generating families. Let $G:\left(\mathbb{R}^{k} \times \mathbb{R}^{n}, \mathbf{0}\right) \longrightarrow(\mathbb{R}, \mathbf{0})$ be a function germ which we call an unfolding of $g(q)=G(q, \mathbf{0})$. We say that $G$ is a Morse family of hypersurfaces if the mapping

$$
\Delta^{*} G=\left(G, \frac{\partial G}{\partial q_{1}}, \ldots, \frac{\partial G}{\partial q_{k}}\right):\left(\mathbb{R}^{k} \times \mathbb{R}^{n}, \mathbf{0}\right) \longrightarrow\left(\mathbb{R} \times \mathbb{R}^{k}, \mathbf{0}\right)
$$


is non-singular, where $(q, x)=\left(q_{1}, \ldots, q_{k}, x_{1}, \ldots, x_{n}\right) \in\left(\mathbb{R}^{k} \times \mathbb{R}^{n}, \mathbf{0}\right)$. In this case we have a smooth $(n-1)$-dimensional submanifold

$$
\Sigma_{*}(G)=\left\{(q, x) \in\left(\mathbb{R}^{k} \times \mathbb{R}^{n}, \mathbf{0}\right) \mid G(q, x)=\frac{\partial G}{\partial q_{1}}(q, x)=\cdots=\frac{\partial G}{\partial q_{k}}(q, x)=0\right\}
$$

and the map germ $\Phi_{G}:\left(\Sigma_{*}(G), \mathbf{0}\right) \longrightarrow P T^{*} \mathbb{R}^{n}$ defined by

$$
\Phi_{G}(q, x)=\left(x,\left[\frac{\partial G}{\partial x_{1}}(q, x): \cdots: \frac{\partial G}{\partial x_{n}}(q, x)\right]\right)
$$

is a Legendrian immersion germ. The fundamental result of Arnol'd-Zakalyukin [2, 34] assets that all Legendrian submanifold germs in $P T^{*} \mathbb{R}^{n}$ are constructed by the above method. We call $G$ a generating family of $\Phi_{G}\left(\Sigma_{*}(G)\right)$. Therefore the wave front of $\Phi_{G}\left(\Sigma_{*}(G)\right)$ is

$$
W\left(\Phi_{G}\right)=\left\{x \in \mathbb{R}^{n} \mid \exists q \in \mathbb{R}^{k} \text { such that } G(q, x)=\frac{\partial G}{\partial q_{1}}(q, x)=\cdots=\frac{\partial G}{\partial q_{k}}(q, x)=0\right\} .
$$

We also write $\mathcal{D}_{G}=W\left(\Phi_{G}\right)$ and call it the discriminant set of $G$.

We now introduce an equivalence relation among Legendrian submanifold germs. Let $i$ : $(L, p) \subset\left(P T^{*} \mathbb{R}^{n}, p\right)$ and $i^{\prime}:\left(L^{\prime}, p^{\prime}\right) \subset\left(P T^{*} \mathbb{R}^{n}, p^{\prime}\right)$ be Legendrian submanifold germs. Then we say that $i$ and $i^{\prime}$ are Legendrian equivalent if there exists a contact diffeomorphism germ $H:\left(P T^{*} \mathbb{R}^{n}, p\right) \longrightarrow\left(P T^{*} \mathbb{R}^{n}, p^{\prime}\right)$ such that $H$ preserves fibers of $\pi$ and that $H(L)=L^{\prime}$.

Since the Legendrian lift $i:(L, p) \subset\left(P T^{*} \mathbb{R}^{n}, p\right)$ is uniquely determined on the regular part of the wave front $W(i)$, we have the following simple but significant property of Legendrian immersion germs:

Proposition A.1. Let $i:(L, p) \subset\left(P T^{*} \mathbb{R}^{n}, p\right)$ and $i^{\prime}:\left(L^{\prime}, p^{\prime}\right) \subset\left(P T^{*} \mathbb{R}^{n}, p^{\prime}\right)$ be Legendrian immersion germs such that the representative of both the regular sets of the projections $\pi \circ i$ and $\pi \circ i^{\prime}$ are dense. Then $i$ and $i^{\prime}$ are Legendrian equivalent if and only if wave front sets $W(i)$ and $W\left(i^{\prime}\right)$ are diffeomorphic as set germs.

This result has been firstly pointed out by Zakalyukin [35]. The assumption in the above proposition is a generic condition for $i$ and $i^{\prime}$.

We can interpret the Legendrian equivalence by using the notion of generating families. We denote $\mathcal{E}_{n}$ the local ring of function germs $\left(\mathbb{R}^{n}, \mathbf{0}\right) \longrightarrow \mathbb{R}$ with the unique maximal ideal $\mathfrak{M}_{n}=$ $\left\{h \in \mathcal{E}_{n} \mid h(\mathbf{0})=0\right\}$. Let $G_{1}, G_{2}:\left(\mathbb{R}^{k} \times \mathbb{R}^{n}, \mathbf{0}\right) \longrightarrow(\mathbb{R}, 0)$ be function germs. We say that $G_{1}$ and $G_{2}$ are $P$ - $\mathcal{K}$-equivalent if there exists a diffeomorphism germ $\Psi:\left(\mathbb{R}^{k} \times \mathbb{R}^{n}, \mathbf{0}\right) \longrightarrow\left(\mathbb{R}^{k} \times \mathbb{R}^{n}, \mathbf{0}\right)$ of the form $\Psi(q, x)=\left(\psi_{1}(q, x), \psi_{2}(x)\right)$ for $(q, x) \in\left(\mathbb{R}^{k} \times \mathbb{R}^{n}, \mathbf{0}\right)$ such that $\Psi^{*}\left(\left\langle G_{1}\right\rangle_{\mathcal{E}_{k+n}}\right)=\left\langle G_{2}\right\rangle_{\mathcal{E}_{k+n}}$. Here $\Psi^{*}: \mathcal{E}_{k+n} \longrightarrow \mathcal{E}_{k+n}$ is the pull back $\mathbb{R}$-algebra isomorphism defined by $\Psi^{*}(h)=h \circ \Psi$.

Let $\bar{G}:\left(\mathbb{R}^{k} \times \mathbb{R}^{n}, \mathbf{0}\right) \longrightarrow(\mathbb{R}, \mathbf{0})$ be a function germ. We say that $\bar{G}$ is a $\mathcal{K}$-versal unfolding of $g=\bar{G} \mid \mathbb{R}^{k} \times\{\mathbf{0}\}$ if for any unfolding $G:\left(\mathbb{R}^{k} \times \mathbb{R}^{m}, \mathbf{0}\right) \longrightarrow(\mathbb{R}, \mathbf{0})$ of $g$ (i.e., $G(q, \mathbf{0})=g(q)$ ), there exists a map germ $\phi:\left(\mathbb{R}^{m}, \mathbf{0}\right) \longrightarrow\left(\mathbb{R}^{n}, \mathbf{0}\right)$ such that $\phi^{*} \bar{G}$ and $G$ are $P$ - $\mathcal{K}$-equivalent, where $\phi^{*} \bar{G}(q, u)=\bar{G}(q, \phi(u))$. For an unfolding $G(t, x)$ of a function $g(t)$ of one-variable, we have the following useful criterion on the $\mathcal{K}$-versal unfoldings in (cf., [4], 6.10): We say that $g$ has an $A_{r}$-singularity at $t_{0}$ if $g^{(p)}\left(t_{0}\right)=0$ for all $1 \leq p \leq r$, and $g^{(r+1)}\left(t_{0}\right) \neq 0$. We have the following lemma 
Lemma A.2. Let $G$ be an unfolding of $g$ and $g(t)$ has an $A_{r}$-singularity $(r \geq 1)$ at $t_{0}$. We denote the $(r-1)$-jet of the partial derivative $\partial G / \partial x_{i}$ at $t_{0}$ by

$$
j^{(r-1)}\left(\frac{\partial G}{\partial x_{i}}\left(t, x_{0}\right)\right)\left(t_{0}\right)=\sum_{j=0}^{r-1} \alpha_{j i}\left(t-t_{0}\right)^{j}
$$

for $i=1, \ldots, n$. Then $G$ is a $\mathcal{K}$-versal unfolding if and only if the $r \times n$ matrix of coefficients $\left(\alpha_{j i}\right)$ has rank $r(r \leq n)$.

It follows from the above lemma that the function germ defined by

$$
t^{r+1}+x_{1} t^{r-1}+x_{2} t^{r-2}+\cdots+x_{r-1} t+x_{r}
$$

is a $\mathcal{K}$-versal unfolding of $g(t)=t^{r+1}$. One of the main results in the theory of Legendrian singularities is the following theorem:

Theorem A.3. Let $G_{1}, G_{2}:\left(\mathbb{R}^{k} \times \mathbb{R}^{n}, \mathbf{0}\right) \longrightarrow(\mathbb{R}, 0)$ be Morse families of hypersurfaces. Then $\Phi_{G_{1}}$ and $\Phi_{G_{2}}$ are Legendrian equivalent if and only if $G_{1}$ and $G_{2}$ are $P$-K-equivalent.

As a corollary of Proposition A.1 and Theorem A.3, we have the following proposition.

Proposition A.4. Let $G_{1}, G_{2}:\left(\mathbb{R}^{k} \times \mathbb{R}^{n}, \mathbf{0}\right) \longrightarrow(\mathbb{R}, 0)$ be Morse families of hypersurfaces. Suppose that both the regular sets of the representative of projections $\pi \circ \Phi_{G_{1}}, \pi \circ \Phi_{G_{2}}$ are dense. Then $\left(W\left(\Phi_{G_{1}}\right), 0\right)$ and $\left(W\left(\Phi_{G_{2}}\right), 0\right)$ are diffeomorphic as set germs if and only if $G_{1}$ and $G_{2}$ are $P$-K-equivalent.

The following Lemma roles the key of the proof for the criteria. Two function germs $g_{i}:(\mathbb{R}, \mathbf{0}) \rightarrow(\mathbb{R}, 0)(i=1,2)$ are $\mathcal{R}$-equivalent if there exist a diffeomorphism germ $\alpha:$ $(\mathbb{R}, 0) \rightarrow(\mathbb{R}, 0)$ such that $\alpha \circ g_{1}=g_{2}$ holds.

Lemma A.5. Let $g:((\mathbb{R} ; t), \mathbf{0}) \rightarrow(\mathbb{R}, 0)$ be a function germ such that $\mathcal{R}$-equivalent to $t^{5}$. If an unfolding $G:\left(\left(\mathbb{R} \times \mathbb{R}^{3} ; t, x, y, z\right), \mathbf{0}\right) \rightarrow(\mathbb{R}, 0)$ of $g$ is a Morse family and a function $\bar{G}(t, x, y, z, w)=G(t, x, y, z)+w t^{3}$ is a $\mathcal{K}$-versal unfolding of $g$, then $P$ - $\mathcal{K}$-equivalent to $t^{5}+$ $x t^{2}+y t+z$.

Proof. Since the condition does not depend on the parameter transformation of $t$, we can assume that $g(t)=t^{5}$. Moreover, since the map $t^{5}+w t^{3}+x t^{2}+y t+z$ is the versal unfolding of $t^{5}$, there is a diffeomorphism $\left(\phi_{1}, \phi_{2}, \phi_{3}, \phi_{4}\right): \mathbb{R}^{4} \rightarrow \mathbb{R}^{4}$ such that $G$ is $P$ - $\mathcal{K}$-equivalent to

$$
t^{5}+\phi_{1}(x, y, z, w) t^{3}+\phi_{2}(x, y, z, w) t^{2}+\phi_{3}(x, y, z, w) t+\phi_{4}(x, y, z, w) .
$$

Since $\bar{G}=G+w t^{3}$ is a $\mathcal{K}$-versal unfolding, and the condition of lemma only depend on the $P$ - $\mathcal{K}$-equivalent class, we can rechoose $(x, y, z)$ such that $\bar{G}$ is $P$ - $\mathcal{K}$-equivalent to

$$
t^{5}+\phi_{1}(x, y, z, w) t^{3}+x t^{2}+y t+z .
$$

Furthermore, since $\bar{G}$ is a versal unfolding and $\partial \phi_{1} / \partial w(\mathbf{0})=0$, we rechoose $w$ such that $\bar{G}$ is $P$-K -equivalent to

$$
t^{5}+(w-h(x, y, z)) t^{3}+x t^{2}+y t+z
$$

for some function $h$. Summerizing up these argument, we can assume that $\bar{G}$ is

$$
\bar{G}_{h}(t, x, y, z):=t^{5}+(w-h(x, y, z)) t^{3}+x t^{2}+y t+z .
$$

We have the following Zakalyukin's lemma 
Lemma A.6. [35, Theorem 1.4] Let $\mathcal{V}:\left(\mathbb{R} \times \mathbb{R}^{4}, \mathbf{0}\right) \rightarrow(\mathbb{R}, 0)$ be a $\mathcal{K}$-versal unfolding of the form

$$
\mathcal{V}(t, x, y, z, w)=t^{5}+w t^{3}+x t^{2}+y t+z
$$

and $\sigma:\left(\mathbb{R}^{4}, \mathbf{0}\right) \rightarrow(\mathbb{R}, 0)$ be a function germ with $(x, y, z, w)$-variables such that $\partial \sigma / \partial w(\mathbf{0}) \neq 0$. Then there exists a differmorphism germ $\Theta:\left(\mathbb{R}^{4}, \mathbf{0}\right) \rightarrow\left(\mathbb{R}^{4}, \mathbf{0}\right)$ such that

$$
\Theta\left(D_{\mathcal{V}}\right)=D_{\mathcal{V}} \text { and } \sigma \circ \Theta(x, y, z, w)=w .
$$

Let us continue to prove of Lemma A.5. We apply Lemma A.6 to $\mathcal{V}$ of (A.1) and $w-$ $h(x, y, z)$. Then there exists a diffeomorphism germ $\Theta$ such that

$$
\Theta\left(D_{\mathcal{V}}\right)=D_{\mathcal{V}} \text { and }(w-h(x, y, z)) \circ \Theta(x, y, z, w)=w
$$

We define a diffeomorphism germ

$$
\Psi(x, y, z, w)=(x, y, z, w-h(x, y, z)),
$$

then it holds that $\Psi^{*} \mathcal{V}=\bar{G}_{h}$. Define a new diffeomorphism germ $\tilde{\Theta}$ by $\tilde{\Theta}=\Psi \circ \Theta$ then we have

$$
\tilde{\Theta}\left(D_{\mathcal{V}}\right)=\Psi \circ \Theta\left(D_{\mathcal{V}}\right)=\Psi\left(D_{\mathcal{V}}\right)=D_{\bar{G}_{h}} .
$$

Hence $D_{\mathcal{V}}$ and $D_{\bar{G}}$ are diffeomorphic. On the other hand, let us define $\pi:\left(\mathbb{R}^{4}, \mathbf{0}\right) \rightarrow(\mathbb{R}, 0)$ by $\pi(x, y, z, w)=w$. Since

$$
\pi \circ \tilde{\Theta}(x, y, z, w)=\pi \circ \Psi \circ \Theta=(w-h(x, y, z)) \circ \Theta=w
$$

we have $\pi \circ \tilde{\Theta}=\pi$. Since the set of regular points of $D_{\mathcal{V}}$ is dense, by the Zakalyukin theorem ([35], see also [28, Appendix]), there exist a diffeomorphism germ $\Xi: \mathbb{R} \times \mathbb{R}^{4} \rightarrow \mathbb{R} \times \mathbb{R}^{4}$ of the form

$$
\begin{aligned}
& \Xi(t, x, y, z, w) \\
& =\left(\xi(t, x, y, z, w), \zeta_{1}(x, y, z, w), \zeta_{2}(x, y, z, w), \zeta_{3}(x, y, z, w), \zeta_{4}(w)\right)
\end{aligned}
$$

such that $\Xi^{*}\left(\langle\mathcal{V}\rangle_{\mathcal{E}_{1+4}}\right)=\left\langle\bar{G}_{h}\right\rangle_{\mathcal{E}_{1+4}}$.

If we restrict the above map to $w=0$, we complete the proof Lemma A.2.

Using these results, we give the criterion of the $A_{4}$-singularity of wave fronts.

Let $f:\left(\mathbb{R}^{2}, \mathbf{0}\right) \rightarrow\left(\mathbb{R}^{3}, \mathbf{0}\right)$ be a front and $\nu$ be the normal vector field of $f$. Let $\mathbf{0}$ is a nondegenerate singular point of $f$. Needless to say, the conditions of Theorem 8.2 do not depend on the choice of coordinates and choice of $\nu$. One can prove the following lemma.

Lemma A.7. One can choose the coordinate systems $(u, v)$ of $\left(\mathbb{R}^{2}, \mathbf{0}\right)$ and $\left(X_{1}, X_{2}, Z\right)$ of $\left(\mathbb{R}^{3}, \mathbf{0}\right)$ satisfying

- $\eta \equiv \partial v$.

- $\quad f(u, v)=\left(f_{1}(u, v), f_{2}(u, v), u\right)$ and $\left(f_{1}\right)_{u}(\mathbf{0})=\left(f_{2}\right)_{u}(\mathbf{0})=0$.

- $\nu(\mathbf{0})=(1,0,0)$.

Under this coordinate system, we prove that if $f: \mathbb{R}^{2} \rightarrow \mathbb{R}^{3}$ satisfies $\eta \lambda=\eta \eta \lambda=0$ and $\eta \eta \eta \lambda \neq 0$ at $\mathbf{0}$ then $f$ at $\mathbf{0}$ is $\mathcal{A}$-equivalent to the cuspidal butterfly. 
Proof of Theorem 8.2. Let us fix a small number $u$ and consider a family of plane curves $\Gamma^{u}(v)=$ $\Gamma(u, v)=\left(f_{1}(u, v), f_{2}(u, v), u\right)$ in the plane $\Pi_{u}=\left\{\left(X_{1}, X_{2}, Z\right) \mid Z=u\right\}$ and show that these are fronts near $\mathbf{0}$. Denote $\nu=\left(\nu_{1}, \nu_{2}, \nu_{3}\right)$ and put

$$
\left[N^{u}(v)\right]=\left[\left(\nu_{1}(u, v), \nu_{2}(u, v), 0\right)\right] .
$$

Then $\left[N^{u}(v)\right]$ is well-defined near $\mathbf{0}$. We put

$$
\gamma(u, v)=\left(f_{1}(u, v), f_{2}(u, v)\right) \text { and } n(u, v)=\left(\nu_{1}(u, v), \nu_{2}(u, v)\right) .
$$

Then, since $\left\langle\gamma^{\prime}(u, v), n(u, v)\right\rangle \equiv 0,(\gamma,[n])$ is an isotropic map for all $u$, where ' denotes $\partial / \partial v$ and $\langle\cdot, \cdot\rangle$ is the canonical inner product of $\mathbb{R}^{3}$. Since $\nu_{3}^{\prime}(\mathbf{0})$, we have $n^{\prime}(\mathbf{0}) \neq \mathbf{0}$. This implies that for each $u$ near $0,(\gamma,[n])$ is a Legendrian immersion germ.

We define two functions $\Psi: \mathbb{R} \times \mathbb{R}^{3} \longrightarrow \mathbb{R}$ and $\psi: \mathbb{R} \longrightarrow \mathbb{R}$ as follows:

$$
\Psi\left(v, X_{1}, X_{2}, Z\right)=n_{1}(Z, v)\left(X_{1}-f_{1}(Z, v)\right)+n_{2}(Z, v)\left(X_{2}-f_{2}(Z, v)\right), \quad \psi(v)=\Psi(v, 0,0,0) .
$$

Then we have $D_{\Psi}=f(U)$. Hence by Lemma A.6 and the arguments in the above, it is sufficient to prove that $\psi$ has an $A_{4}$-singularity and $\Psi$ satisfies the conditions of Lemma A.5. In the following context, we put $Z=u$.

Lemma A.8. It holds that $f^{\prime}(\mathbf{0})=f^{\prime}(\mathbf{0})=f^{\prime \prime \prime}(\mathbf{0})=\mathbf{0}, f^{\prime \prime \prime \prime}(\mathbf{0}) \neq \mathbf{0}$ and $(\bar{f})^{\prime}(\mathbf{0})=(\bar{f})^{\prime \prime}(\mathbf{0})=$ $(\bar{f})^{\prime \prime \prime}(\mathbf{0})=\mathbf{0},(\bar{f})^{\prime \prime \prime \prime}(\mathbf{0}) \neq \mathbf{0}$.

Proof. Since $\partial_{v}$ is the null vector field, so that we have $f^{\prime}(\mathbf{0})=\mathbf{0}$ and $S(f)=\left\{f_{v}=\mathbf{0}\right\}$. By $\eta \lambda=0$, since $\left(\partial_{v}=\right) \eta_{0} \in T_{0} S(f)$, it holds that $f^{\prime \prime}(\mathbf{0})=\mathbf{0}$. Furthremore, by $\lambda^{\prime \prime}(\mathbf{0})=0$ and $f^{\prime}(\mathbf{0})=f^{\prime \prime}(\mathbf{0})=\mathbf{0}$, we have $\lambda^{\prime \prime}(\mathbf{0})=\operatorname{det}\left(f_{u}, f^{\prime \prime \prime}, \nu\right)(\mathbf{0})$. Hence it holds that $f^{\prime \prime \prime}(\mathbf{0}) \in$ $\operatorname{span}\left\{f_{u}(\mathbf{0}), \nu(\mathbf{0})\right\}$.

On the other hand, we have $\left\langle f_{u}, f^{\prime \prime \prime}\right\rangle(\mathbf{0})=\langle(0,0,1),(*, *, 0)\rangle=0$. Differenciating $\left\langle\nu, f^{\prime}\right\rangle=$ 0 , we have $\left\langle\nu, f^{\prime \prime}\right\rangle=\left\langle\nu, f^{\prime}\right\rangle^{\prime}-\left\langle\nu^{\prime}, f^{\prime}\right\rangle$ and $\left\langle\nu, f^{\prime \prime \prime}\right\rangle=\left\langle\nu, f^{\prime \prime}\right\rangle^{\prime}-\left\langle\nu^{\prime}, f^{\prime \prime}\right\rangle$. Hence $\left\langle\nu, f^{\prime \prime}\right\rangle \equiv 0$ holds on $S(f)$. Since $\eta_{0} \in T_{0} S(f)$, it holds that $\left\langle\nu, f^{\prime \prime}\right\rangle^{\prime}(\mathbf{0})=0$ and $\left\langle\nu, f^{\prime \prime \prime}\right\rangle(\mathbf{0})=0$. Thus we have $f^{\prime \prime \prime}(\mathbf{0})=0$.

Since $\lambda^{\prime \prime \prime}(\mathbf{0}) \neq 0$ and $f^{\prime}(\mathbf{0})=f^{\prime \prime}(\mathbf{0})=f^{\prime \prime \prime}(\mathbf{0})=\mathbf{0}$, it holds that $0 \neq \lambda^{\prime \prime \prime}(\mathbf{0})=\operatorname{det}\left(f_{u}, f^{\prime \prime \prime \prime \prime}, \nu\right)(\mathbf{0})$. In particular, $f^{\prime \prime \prime \prime}(\mathbf{0}) \neq \mathbf{0}$ holds.

To prove Theorem 8.2, firstly we show that $\psi$ has the $A_{4}$-singularity at $\mathbf{0}$. Differenciating $\left\langle(\bar{f})^{\prime}, n\right\rangle \equiv 0$ and by Lemma A.8, we have $\left\langle(\bar{f})^{\prime \prime \prime \prime}, n\right\rangle(\mathbf{0})=0$ and $4\left\langle(\bar{f})^{\prime \prime \prime \prime \prime}, n^{\prime}\right\rangle(\mathbf{0})+$ $\left\langle(\bar{f})^{\prime \prime \prime \prime \prime \prime}, n\right\rangle(\mathbf{0})=0$.

By these formulas and Lemma A.8, we have $\psi^{\prime}(0)=\psi^{\prime \prime}(0)=\psi^{\prime \prime \prime}(0)=0, \psi^{\prime \prime \prime \prime}(0)=$ $-\left\langle n,(\bar{f})^{\prime \prime \prime \prime \prime}\right\rangle(\mathbf{0})=0$ and $\psi^{\prime \prime \prime \prime \prime}(0)=-\left\langle n^{\prime},(\bar{f})^{\prime \prime \prime \prime \prime}\right\rangle(\mathbf{0})$.

On the other hand, since $n, n^{\prime}$ is linearly independent at $\mathbf{0}$ and $\left\langle n,(\bar{f})^{\prime \prime \prime \prime}\right\rangle(\mathbf{0})=0$, we have

$$
\left\langle n^{\prime},(\bar{f})^{\prime \prime \prime \prime \prime}\right\rangle(\mathbf{0}) \neq 0 \Longleftrightarrow(\bar{f})^{\prime \prime \prime \prime}(\mathbf{0}) \neq \mathbf{0} \Longleftrightarrow f^{\prime \prime \prime \prime}(\mathbf{0}) \neq \mathbf{0} .
$$

Hence $\psi$ has the $A_{4}$ singularity at $\mathbf{0}$.

Next, we show that $\left(\Psi, \Psi^{\prime}, \Psi^{\prime \prime}\right)$ is non-singular. If this is satisfied, $\Psi$ satisfies the condition of Lemma A.5 namely, $\Psi$ is a Morse family and $\Psi\left(v, X_{1}, X_{2}, u\right)+w v^{3}$ is a $\mathcal{K}$-versal unfolding of $\psi$. Remark that the discriminant set of an unfolding $t^{5}+x t^{2}+y t+z$ of a function $t^{5}$ is diffeomorphic to the image of the canonical cuspidal butterfly $(u, v) \mapsto\left(u, 5 v^{4}+2 u v, 4 v^{5}+u v^{2}-u^{2}\right)$ at $\mathbf{0}$ as set 
germs. Therefore by Proposition A.4 and Lemma A.5, we can show that $f$ at $\mathbf{0}$ is $\mathcal{A}$-equivalent to the cuspidal butterfly.

Since $\Psi_{X}(\mathbf{0})=-n_{1}(\mathbf{0}), \Psi_{Y}(\mathbf{0})=-n_{2}(\mathbf{0})$ and $\Psi_{u}=\sum_{i=1,2}\left\langle\left(n_{i}\right)_{u}, X_{i}-f_{i}\right\rangle-\left\langle n_{i},\left(f_{i}\right)_{u}\right\rangle$, it holds that $\Psi_{u}(\mathbf{0})=0$. By a direct calculation, we have $\Psi_{X}^{\prime}(\mathbf{0})=-n_{1}^{\prime}(\mathbf{0}), \Psi_{Y}^{\prime}(\mathbf{0})=-n_{2}^{\prime}(\mathbf{0})$ and $\Psi_{u}^{\prime}=\sum_{i=1,2}\left\langle\left(n_{i}\right)_{u}^{\prime}, X_{i}-f_{i}\right\rangle-\left\langle n_{i}, f^{\prime}\right\rangle-\left\langle n_{i}^{\prime},\left(f_{i}\right)_{u}\right\rangle-\left\langle n_{i},\left(f_{i}\right)_{u}^{\prime}\right\rangle$. Since $\left\langle n_{i},\left(f_{i}\right)_{u}^{\prime}\right\rangle=\left\langle n,(\bar{f})^{\prime}\right\rangle_{u}-$ $\left\langle n_{u},(\bar{f})^{\prime}\right\rangle=0$ holds at $\mathbf{0}$, we have $\Psi_{u}^{\prime}(\mathbf{0})=0$.

Thus it is sufficient to prove that the matrix

$$
\left(\begin{array}{lll}
\partial(\Psi, & \Psi^{\prime}, & \left.\Psi^{\prime \prime}\right) / \partial t \\
\partial(\Psi, & \Psi^{\prime}, & \left.\Psi^{\prime \prime}\right) / \partial X_{1} \\
\partial(\Psi, & \Psi^{\prime}, & \left.\Psi^{\prime \prime}\right) / \partial X_{2} \\
\partial(\Psi, & \Psi^{\prime}, & \left.\Psi^{\prime \prime}\right) / \partial u
\end{array}\right)(\mathbf{0})=\left(\begin{array}{ccc}
0 & 0 & 0 \\
n_{1} & n_{1}^{\prime} & * \\
n_{2} & n_{2}^{\prime} & * \\
0 & 0 & \Psi_{u}^{\prime \prime}
\end{array}\right)(\mathbf{0})
$$

is of full rank. Hence we show that $\Psi_{u}^{\prime \prime}(\mathbf{0}) \neq 0$.

Differenciating $\left\langle n,(\bar{f})^{\prime}\right\rangle \equiv 0$ by $u$ and $v$, and by Lemma A.8, we have

$$
\left\langle n,(\bar{f})_{u}^{\prime \prime}\right\rangle(\mathbf{0})=\left\langle n^{\prime},(\bar{f})_{u}^{\prime}\right\rangle(\mathbf{0})
$$

Differentiating $\Psi$ by $u$ and $v$ two times, and by Lemma A.8 and (A.2), we have

$$
\Psi_{u}^{\prime \prime}(\mathbf{0})=-\left\langle n^{\prime},(\bar{f})_{u}^{\prime}\right\rangle(\mathbf{0})
$$

On the other hand, since $\left\langle n,(\bar{f})_{u}^{\prime}\right\rangle(\mathbf{0})=0$,

$$
\left\langle n^{\prime},(\bar{f})_{u}^{\prime}\right\rangle(\mathbf{0}) \neq 0 \Longleftrightarrow(\bar{f})_{u}^{\prime}(\mathbf{0}) \neq 0 \Longleftrightarrow f_{u}^{\prime}(\mathbf{0}) \neq 0
$$

holds. By $\lambda_{u}(\mathbf{0}) \neq 0$ and $f_{v}(\mathbf{0})=\mathbf{0}$, we have

$$
0 \neq \lambda_{u}(\mathbf{0})=\operatorname{det}\left(f_{u}, f_{u}^{\prime}, \nu\right)(\mathbf{0})
$$

In particular, $f_{u}^{\prime}(\mathbf{0}) \neq \mathbf{0}$ holds. This implies the desired result.

The converse pert of the theorem is obvious since the conditions and assertions of Theorem 8.2 are independent of the choice of coordinates and choce of $\nu$, and the canonical $A_{4}$ singularity satisfies the condition of theorem.

Remark that since $\mathbf{0}$ is a non-degenerate singular point, we have the parameterization $\gamma(t)$ of $S(f)$. Take the null vector field on $\gamma$ as $\eta(t)$. Define a function of $t$ by

$$
\mu(t)=\operatorname{det}\left(\gamma^{\prime}(t), \eta(t)\right)
$$

One can easily show that $\mu(0)=\mu^{\prime}(0)=0$ and $\mu^{\prime \prime}(0) \neq 0$ and $\eta \lambda(\mathbf{0})=\eta \eta \lambda(\mathbf{0})=0$ and $\eta \eta \eta \lambda(\mathbf{0}) \neq 0$ are equivalent, as a corollary, the following assertion holds.

Corollary A.9. A front germ $f$ at $\mathbf{0}$ is $\mathcal{A}$-equivalent to the $A_{4}$-singularity if and only if $\mathbf{0}$ is a non-degenerate singular point and $\mu(0)=\mu^{\prime}(0)=0$ but $\mu^{\prime \prime}(0) \neq 0$ holds. 


\section{References}

[1] R. Aiyama and K Akutagawa, Kenmotus-Bryant type representation formulas for constant mean curvature surfaces in $H^{3}\left(-c^{2}\right)$ and $S_{1}^{3}\left(c^{2}\right)$, Math. global anal. geom. (1) 17 (1998), $49-75$.

[2] V. I. Arnol'd, S. M. Gusein-Zade and A. N. Varchenko, Singularities of Differentiable Maps vol. I. Birkhäuser (1986)

[3] J. A. Aledo and J. M. Espinar, A conformal representation for linear Weingarten surfaces in the de Sitter space, Journal of geom. and phys., 57 (2007), 1669-1677.

[4] J. W. Bruce and P. J. Giblin, Curves and singularities (second edition), Cambridge University press (1992).

[5] M. Buosi, S. Izumiya and M. A. Ruas, Total absolute horospherical curvature of submanifolds in Hyperbolic space, to appear in Advances in Geometry

[6] M. Buosi, S. Izumiya and M. A. Ruas, Horo-tight spheres in Hyperbolic space, preprint (2009)

[7] D. E. Blair, Contact Manifolds in Riemannian Geometry. Lecture Notes in Mathematics 509 Springer (1976)

[8] R. L. Bryant, Surfaces of mean curvature one in hyperbolic space )) in Théorie des variétés minimales et applications (Palaiseau, 1983-1984), Astérisque No. 154-155 (1987), 12, 321$347,353(1988)$

[9] T. E. Cecil, Lie Sphere Geometry. Universitetext, Springer (1992)

[10] L. Chen and S. Izumiya, A mandala of Legendrian dualities for pseudo-spheres in semiEuclidean space. Proceedings of the Japan Academy, 85 Ser. A (2009), 49-54

[11] C. L. Epstein, Envelopes of Horospheres and Weingarten Surfaces in Hyperbolic 3-Space. Preprint, Princeton Univ., (1984)

[12] J. A. Galvez, A. Martinez and F. Milan, Complete linear Weingarten surfaces of Bryant type. A Plateau problem at infinity, Trans AMS, 356, 9 (2004), 3405-3428.

[13] S. Fujimori, K. Saji, M. Umehara and K. Yamada, Singularities of maximal surfaces, Math. Z. 259 (4) (2008), 827-848.

[14] A. B. Givental', Singular Lagrangian manifolds and their Lagrangian mappings, Itogi Nauki Tekh., Ser. Sovrem. Prob. Mat., 33, 1988, 55-112.

[15] G. Ishikawa, Infinitesimal deformations and stability of singular Legendre submanifolds, Asian J. Math. 9 (2005), no. 1, 133-166.

[16] S. Izumiya and N. Takeuchi, Singularities of ruled surfaces in $R^{3}$, Math. Proc. Camb. Philos. Soc. 130 (2001), no. 1, 1-11.

[17] S. Izumiya, D-H. Pei and T. Sano, Singularities of hyperbolic Gauss maps. Proceedings of the London Mathematical Society 86 (2003), 485-512 
[18] S. Izumiya and M. C. Romero Fuster, The horospherical Gauss-Bonnet type theorem for hypersurfaces in hyperbolic space, Journal of Mathematical Society of Japan 58, (2006), 965984

[19] S. Izumiya, D-H. Pei, M. C. Romero-Fuster and M. Takahashi, On the horospherical ridges of submanifolds of codimension 2 in Hyperbolic n-space, Bull. Braz. Math. Soc. 35 (2) (2004), $177-198$

[20] S. Izumiya, D-H. Pei and M. Takahashi, Singularities of evolutes of hypersurfaces in hyperbolic space, Proceedings of the Edinburgh Mathematical Society 47 (2004), 131-153

[21] S. Izumiya, D-H. Pei and M. C. Romero-Fuster, The horospherical geometry of surfaces in Hyperbolic 4-space, Israel Journal of Mathematics 154, (2006), 361-379

[22] S. Izumiya, K. Saji and M. Takahashi, Horospherical flat surfaces in hyperbolic 3-space, to appear in Journal of Mathematical Society of Japan, 62 (2010)

[23] S. Izumiya, K. Saji and N. Takeuchi, Circular surfaces, Adv. Geom. 7 (2007), no. 2, 295-313.

[24] S. Izumiya, Differential Geometry from the viewpoint of Lagrangian or Legendrian singularity theory, in Singularity Theory, Proceedings of the 2005 Marseille Singularity School and Conference, ed., D. Chéniót et al., World Scientific (2007), 241-275

[25] S. Izumiya, Legendrian dualities and spacelike hypersurfaces in the lightcone. Moscow Mathematical Journal 9 (2009), 325-357

[26] S. Izumiya and H. Yıldırım, Extensions of the mandala of Legendrian dualities for pseudospheres in Lorentz-Minkowski space. Preprint (2009)

[27] M. Kokubu and M. Umehara, Orientability of linear Weingarten surfaces, spacelike CMC-1 surfaces and maximal surfaces, preprint.

[28] M. Kokubu, W. Rossman, K. Saji, M. Umehara and K. Yamada, Singularities of flat fronts in hyperbolic 3-space, Pacific J. Math. 221 (2005), no. 2, 303-351.

[29] B. O’Neill, Semi-Riemannian Geometry, Academic Press, New York (1983)

[30] I. Porteous, The normal singularities of submanifold. J. Diff. Geom., vol 5, (1971), 543-564

[31] M. C. Romero Fuster, Sphere stratifications and the Gauss map. Proceedings of the Royal Soc. Edinburgh, 95A (1983), 115-136

[32] K. Saji, M. Umehara and K. Yamada, $A_{k}$ singularities of wave fronts, to appear in Mathematical Proceedings Cambridge Philosophical Society.

[33] O. P. Shcherbak, Projectively dual space curves and Legendre singularities, Sel. Math. Sov. 5, no.4, (1986) 391-421.

[34] V. M. Zakalyukin, Lagrangian and Legendrian singularities. Funct. Anal. Appl., 10 (1976), $23-31$

[35] V. M. Zakalyukin, Reconstructions of fronts and caustics depending one parameter and versality of mappings. J. Sov. Math., 27 (1984), 2713-2735 


\section{S. IZUMIYA,}

Department of Mathematics, Faculty of Science, Hokkaido University, Sapporo 060-0810, Japan and

K. SAJI,

Department of Mathematics, Faculty of Education, Gifu University, Yanagido 1-1, Gifu, 501-1193, Japan

e-mail : izumiyalmath.sci.hokudai.ac.jp,

e-mail : ksajilgifu-u.ac.jp 